\title{
AN OPTIMIZATION BASED COUPLING METHOD FOR MULTISCALE PROBLEMS*
}

\author{
ASSYR ABDULLE ${ }^{\dagger}$, ORANE JECKER $^{\dagger}$, AND ALEXANDER SHAPEEV ${ }^{\ddagger}$
}

\begin{abstract}
A new multiscale coupling method is proposed for elliptic problems with highly oscillatory coefficients with a continuum of scales in a subset of the computational domain and scale separation in complementary regions of the computational domain. A discontinuous Galerkin (DG) finite element heterogeneous multiscale method (FE-HMM) is used in the region with scale separation, while a continuous standard finite element method is used in the region without scale separation. The use of a DG-FE-HMM method allows for a flexible meshing of the different models in the overlapping region. The unknown boundary conditions at the interfaces are obtained by minimizing the error of the two models in the overlapping region. We prove the well-posedness of both the continuous and discrete coupling problems and establish convergence of the multiscale method towards the fine scale solution. Since in the region with scale separation we obtain an approximation at a cost independent of the smallest scale in the problem, the computational cost of the multiscale method is significantly smaller than a fine scale solver over the whole computational domain, while the algorithm allows us to treat situations for which standard numerical homogenization methods do not apply.
\end{abstract}

Key words. optimization based coupling, virtual control, homogenization, multiscale problem, HMM, discontinuous Galerkin

AMS subject classifications. 65N30, 35J15, 35B27, 49J20

DOI. $10.1137 / 15 \mathrm{M} 105389 \mathrm{X}$

1. Introduction. Partial differential equations (PDEs) with multiple scales are used to model a wide range of physical systems with numerous applications, ranging from material and natural sciences to problems in engineering or biology. When the ratio of the smallest scale in the problem to the size of the computational domain is very large, the numerical approximation of such problems with classical numerical methods can become computationally prohibitive as the smallest scales in the problem have to be resolved, leading to discretization with a very large number of degrees of freedom. Numerous multiscale methods have been developed in the past decade. Without attempting to be exhaustive, we recall two important approaches that we will contrast later with the new multiscale method proposed and analyzed in this paper. We will focus on linear elliptic problems, but note that some methods described below have been proposed also for other types of PDEs.

We first mention methods based on coarse oscillatory basis functions that encode the high variation of the data in the multiscale PDE. In this class of methods we have, for example, the multiscale finite element method (see the references in, e.g., [22]) and the recently proposed local orthogonal decomposition (LOD) (see [32, 27]). In principle these methods can be applied to problems with general coefficients (e.g., without structural assumption on the coefficients), and convergence has, indeed, been proved for rough coefficients for the LOD in [32, 27]. While these methods are quite

\footnotetext{
* Received by the editors December 28, 2015; accepted for publication (in revised form) July 27, 2016; published electronically November 3, 2016.

http://www.siam.org/journals/mms/14-4/M105389.html

Funding: The work of the first and second authors was supported in part by the Swiss National Science Foundation.

†École Polytechnique Fédérale de Lausanne, 1015 Lausanne, Switzerland (assyr.abdulle@epfl.ch, orane.jecker@epfl.ch).

¥Skolkovo Institute of Science and Technology, Moscow 143026, Russia (alexander@shapeev.com).
} 
general, they also come with a high computational cost to precompute the coarse basis functions, as the original fine scale problem has to be solved on localized coarse elements whose union is a partition of the computational domain of interest.

The next class of multiscale methods that we mention are methods supplementing macroscopic data (computed through microcomputations) for the solution of an effective equation solved by a macroscopic solver. This approach, widely used by engineers (see, e.g., the references in [23]), has been developed into a general framework in the heterogeneous multiscale method (HMM) $[20,2]$. When finite element methods (FEMs) are used (at the micro- and macroscales), these methods are called finite element heterogeneous multiscale methods (FE-HMMs). The theoretical justification of these methods is that of the homogenization theory: given a family of PDEs indexed by a parameter $\varepsilon$, the theory of $H$-convergence establishes the convergence of a subsequence of solutions to an effective PDE under quite general assumptions (e.g., boundedness and ellipticity of the diffusion tensor of an elliptic problem and righthand side in the dual of the Hilbert space considered in the weak formulation). In a numerical approach such as the FE-HMM, the microcomputations are usually done in sampling domains of size much smaller than the mesh width used for the macroscopic solver. Hence, to extract the effective data, a computational cost independent of the small scales can be achieved when, indeed, the small scales can be localized, i.e., when the problem features scale separation. Rigorous convergence analysis has been established for locally periodic coefficients or random stationary coefficients $[1,21,3,2]$.

In this paper we are interested in problems in which the scales are separated in a subset of the computational domain with possibly a continuum of scales in the complementary domain. Our aim is to couple numerical homogenization methods such as the FE-HMM in part of the computational domain with a fine scale solver. Such problems arise in many situations, for example, heterogeneous composite materials whose effective properties can be well captured by assuming a (locally) periodic microstructure that might not, however, be valid near defects. In our modeling the smallest scale is still supposed to be discretized at the continuum level, but for some applications an atomistic scale should be considered.

Algorithms that couple numerical homogenization methods with a fine scale solver have appeared in the literature. We mention the goal-oriented method [36], in which the unknown boundary conditions for the fine scale subregions are provided by a precomputed homogenized solution. Recently in [9], the authors propose a local-global solution based on the $L^{2}$ projection of the homogenized solution onto the solutions of fine scale local problems.

In this paper we propose and analyze a new coupling strategy inspired by virtual control methods pioneered in [26, 31, 24] (see also [19] for recent developments). Our method also shares some similarities with the recent works on atomistic-to-continuum coupling [37] and the coupling of local and nonlocal diffusion models [16]. The method that we propose relies on a decomposition of the computational domain $\Omega$ into a region without scale separation $\omega$ where the homogenized model is not valid, an overlapping region $\omega_{0}$ where both the fine scale and the homogenized models are valid, and a region $\omega_{2}$ where the homogenized solution adequately describes the physical problem. Thus, we decompose the domain into a family of overlapping domains and introduce virtual (interface) controls as boundary conditions. The interface controls will act as unknown traces or fluxes, and the problem is reformulated as a minimization problem with state equations as constraints. The optimal boundary controls of two overlapping domains are found by a heterogeneous optimization problem that is based on minimizing the discrepancy between the two models on the overlapping region. It is shown that by 
using a Caccioppoli inequality, the minimization can be performed for an $L^{2}$ norm. As in the region with scale separation an energy approximation towards the fine scale problems can also be obtained through the use of a locally periodic corrector we also obtain an $H^{1}$-convergence rate towards the fine scale solution over the whole computational domain. In order to allow flexibility in the mesh used in the coarse and fine scale regions, we use the discontinuous Galerkin (DG) FE-HMM [4] for the numerical homogenization. The method analyzed in this paper was first announced in [5]. In this paper we give a more general framework for the method presented in [5] and offer the first full analysis for both the continuous and the discrete coupling algorithms.

We finally compare our method with the recently developed numerical homogenization of periodic microstructure with a defect proposed in [11]. There, the highly oscillatory coefficient is assumed to be the sum of a periodic function and a localized perturbation. The goal is to compute an approximation of the fine scale solution that relies on homogenization but uses a nonperiodic corrector on a domain that accounts for the defect. We will further compare these two approaches, each of which is interesting in its own right, in our numerical experiments.

The outline of this article is as follows. In section 2 we describe our optimization based multiscale method and prove the well-posedness of the optimization problem. A priori error estimates of the continuous version of the optimization algorithm are proved in section 3, while the fully discrete optimization based method is described in section 4 . In section 5 we state and prove fully discrete error estimates between the numerical solution of the multiscale optimization based method and the fine scale solution. Numerical experiments that verify the theoretical convergence rates and comparisons with other coupling strategies are provided in section 6 .

Notation. In what follows, $C>0$ is used to denote a generic constant independent of $\varepsilon$. We consider the usual Sobolev space $H^{1}(\Omega)=\left\{u \in L^{2}(\Omega)\left|D^{r} u \in L^{2}(\Omega),\right| r \mid \leq\right.$ $1\}$, where $r \in \mathbb{N}^{d},|r|=r_{1}+\cdots+r_{d}$, and $D^{r}=\partial_{1}^{r_{1}} \ldots \partial_{d}^{r_{d}}$. The notation $|\cdot|$ stands for the standard Euclidean norm in $\mathbb{R}^{d}$. Let $Y$ denote the unit cube $(0,1)^{d}$, and define $W_{\mathrm{per}}^{1}(Y):=\left\{v \in H_{\mathrm{per}}^{1}(Y) \mid \int_{Y} v \mathrm{~d} y=0\right\}$, where the set $H_{\mathrm{per}}^{1}(Y)$ is the closure of $\mathcal{C}_{\text {per }}^{\infty}(Y)$ in the $H^{1}$ norm.

2. Optimization based method. Let $\Omega$ be a convex, polygonal domain in $\mathbb{R}^{d}, d=1,2,3$, with a boundary $\Gamma=\Gamma_{D} \cup \Gamma_{N}$, where Dirichlet conditions are imposed on $\Gamma_{D}$ and Neumann conditions on $\Gamma_{N}$. Further, assume that $\Gamma_{D} \cap \Gamma_{N}=\emptyset$ and that $\Gamma_{D}$ has positive measure. Let $f \in L^{2}(\Omega), g_{D} \in H^{1 / 2}\left(\Gamma_{D}\right)$, and $g_{N} \in L^{2}\left(\Gamma_{N}\right)$, and consider the following second order elliptic problem:

$$
\begin{aligned}
-\operatorname{div}\left(a^{\varepsilon} \nabla u^{\varepsilon}\right) & =f & & \text { in } \Omega, \\
u^{\varepsilon} & =g_{D} & & \text { on } \Gamma_{D}, \\
n \cdot\left(a^{\varepsilon} \nabla u^{\varepsilon}\right) & =g_{N} & & \text { on } \Gamma_{N},
\end{aligned}
$$

where the $a^{\varepsilon} \in\left(L^{\infty}(\Omega)\right)^{d \times d}$ are highly oscillatory, bounded coefficients with scale separation only in some subregions of $\Omega$. Further, $a^{\varepsilon}$ is uniformly elliptic; that is, there exists $0<\alpha \leq \beta$ such that

$$
\alpha|\xi|^{2} \leq a^{\varepsilon}(x) \xi \cdot \xi, \quad\left|a^{\varepsilon}(x) \xi\right| \leq \beta|\xi| \quad \forall \xi \in \mathbb{R}^{d} \text {, for a.e. } x \in \mathbb{R} .
$$

Thanks to the Lax-Milgram lemma, problem (2.1) is well-posed.

Let $\omega$ denote a subregion of $\Omega$ in which there is no scale separation. Hence we denote by $\omega_{2}:=\Omega \backslash \bar{\omega}$ the domain where we will apply the classical homogenization. The 

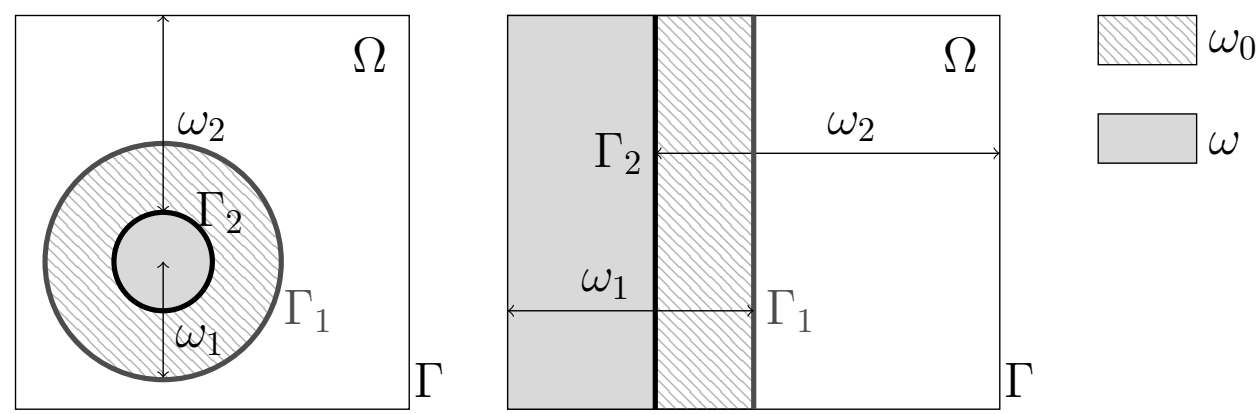

FIG. 1. Illustration of two scenarios for the domain $\Omega$ and its subregions.

fine scale solver will be applied on a domain $\omega_{1}$, slightly larger than $\omega, \omega_{1} \supset \supset$, and the overlap is denoted by $\omega_{0}:=\omega_{1} \cap \omega_{2}$. Figure 1 illustrates possible domain decompositions. Assume that the tensor $a^{\varepsilon}$ is given by $a^{\varepsilon}(x)=a_{\omega}^{\varepsilon}(x) \mathbb{1}_{\omega}(x)+a_{2}^{\varepsilon}(x) \mathbb{1}_{\omega_{2}}(x)$, where $\mathbb{1}_{\omega}$ denotes the characteristic function associated to the subdomain $\omega$. Further, assume that the tensor $a_{2}^{\varepsilon}$ has scale separation, e.g., $a_{2}(x, x / \varepsilon)$, and is locally periodic in the fast variable. Following the homogenization theory, a tensor $a_{2}^{0}$ can be derived from $a_{2}^{\varepsilon}$. On the contrary, in the tensor $a_{\omega}^{\varepsilon}$, the scales are not well separated, which prevents the use of numerical homogenization methods. The heterogeneities can also be present in the right-hand side $f$, and following homogenization theory, the smooth part of $f$ converges to a function $f^{0}$ when the size of the heterogeneities goes to zero; see $[15]$.

Let $\Gamma_{1}=\partial \omega_{1} \backslash \Gamma$ and $\Gamma_{2}=\partial \omega_{2} \backslash \Gamma$ be Lipschitz continuous boundaries. We consider the following minimization problem: find $u_{1}^{\varepsilon} \in H^{1}\left(\omega_{1}\right)$ and $u_{2}^{0} \in H^{1}\left(\omega_{2}\right)$ such that $\frac{1}{2}\left\|u_{1}^{\varepsilon}-u_{2}^{0}\right\|_{\mathrm{L}^{2}\left(\omega_{0}\right)}^{2}$ is minimized under the following constraints for $i=1,2$ :

$$
\begin{array}{rlrl}
-\operatorname{div}\left(a_{i} \nabla u_{i}\right) & =f & & \text { in } \omega_{i}, \\
u_{i}=\theta_{i} & & \text { on } \Gamma_{i}, \\
u_{i}=g_{D} & & \text { on } \partial \omega_{i} \cap \Gamma_{D}, \\
n_{i} \cdot\left(a_{i} \nabla u_{i}\right) & =g_{N} & & \text { on } \partial \omega_{i} \cap \Gamma_{N},
\end{array}
$$

where the boundary conditions $\theta_{i}$, which we refer to as virtual controls, are to be determined. Here and in what follows, we will sometimes use the short-hand notation

$$
\begin{array}{ll}
a_{1}=a_{1}^{\varepsilon}=a_{\omega}^{\varepsilon} \mathbb{1}_{\omega}+a_{2}^{\varepsilon} \mathbb{1}_{\omega_{0}}, & u_{1}=u_{1}^{\varepsilon}, \\
a_{2}=a_{2}^{0}, & u_{2}=u_{2}^{0},
\end{array}
$$

and $u_{i}\left(\theta_{i}\right)$ to emphasize the dependency on $\theta_{i}$. One could also consider Neumann boundary controls instead of Dirichlet controls and follow the theory with some adjustments.

The strategy is to solve a minimization problem in a space of admissible controls, where the cost function to minimize is

$$
J\left(\theta_{1}, \theta_{2}\right)=\frac{1}{2}\left\|u_{1}^{\varepsilon}\left(\theta_{1}\right)-u_{2}^{0}\left(\theta_{2}\right)\right\|_{L^{2}\left(\omega_{0}\right)}^{2} .
$$

The existence and uniqueness of the solution will be proved following the method of Lions [29]. 
Following the virtual control method exposed in [24], we split the solutions into two parts as

$$
u_{1}^{\varepsilon}\left(\theta_{1}\right)=u_{1,0}^{\varepsilon}+v_{1}^{\varepsilon}\left(\theta_{1}\right), \quad u_{2}^{0}\left(\theta_{2}\right)=u_{2,0}^{0}+v_{2}^{0}\left(\theta_{2}\right),
$$

where $u_{1,0}^{\varepsilon}$ and $u_{2,0}^{0}$ are independent of the controls and are defined as in (2.5). The functions $\left(v_{1}^{\varepsilon}, v_{2}^{0}\right)$ are called the state variables and satisfy, for $i=1,2$,

$$
\left\{\begin{array}{rlrl}
-\operatorname{div}\left(a_{i} \nabla v_{i}\right) & =0 & & \text { in } \omega_{i}, \\
v_{i}=\theta_{i} & & \text { on } \Gamma_{i}, \\
v_{i} & =0 & & \text { on } \partial \omega_{i} \cap \Gamma_{D}, \\
n_{i} \cdot\left(a_{i} \nabla v_{i}\right) & =0 & & \text { on } \partial \omega_{i} \cap \Gamma_{N},
\end{array}\right.
$$

where $v_{1}=v_{1}^{\varepsilon}$ and $v_{2}=v_{2}^{0}$.

The space of admissible Dirichlet controls on $\Gamma_{i}, i=1,2$, is defined by

$$
\begin{array}{r}
\mathcal{U}_{i}^{D}=\left\{\mu_{i} \in H^{1 / 2}\left(\Gamma_{i}\right) \mid \exists u \in H^{1}\left(\omega_{i}\right), u=\mu_{i} \text { on } \Gamma_{i}, u=0 \text { on } \partial \omega_{i} \cap \Gamma_{D},\right. \\
\text { and } \left.n_{i} \cdot\left(a_{i} \nabla u\right)=0 \text { on } \partial \omega_{i} \cap \Gamma_{N}\right\} .
\end{array}
$$

For simplicity, we set $\mathcal{U}:=\mathcal{U}_{1}^{D} \times \mathcal{U}_{2}^{D}$. We define for $i=1,2$

$$
\begin{aligned}
H_{D}^{1}\left(\omega_{i}\right) & =\left\{u \in H^{1}\left(\omega_{i}\right) \mid u=0 \text { on } \partial \omega_{i} \cap \Gamma_{D}\right\}, \\
H_{D, \Gamma_{i}}^{1}\left(\omega_{i}\right) & =\left\{u \in H^{1}\left(\omega_{i}\right) \mid u=0 \text { on } \partial \omega_{i} \cap \Gamma_{D} \text { and } \Gamma_{i}\right\} .
\end{aligned}
$$

Let $\gamma_{D}: H^{1}(\Omega) \rightarrow H^{1 / 2}\left(\Gamma_{D}\right)$ denote a linear continuous map, called the trace map. As $g_{D}$ is in $H^{1 / 2}\left(\Gamma_{D}\right)$, there exists $R_{g_{D}} \in H^{1}(\Omega)$, called a lifting of the boundary data $g_{D}$, such that $\gamma_{D}\left(R_{g_{D}}\right)=g_{D}$. Further, there exists a constant $C(\Omega)$ depending on $\Omega$ such that

$$
\left\|R_{g_{D}}\right\|_{\mathrm{H}^{1}(\Omega)} \leq C(\Omega)\left\|g_{D}\right\|_{\mathrm{H}^{1 / 2}\left(\Gamma_{D}\right)} .
$$

The function $u_{i, 0} \in H_{D, \Gamma_{i}}^{1}\left(\omega_{i}\right)$ satisfies, for all test functions $w \in H_{D, \Gamma_{i}}^{1}\left(\omega_{i}\right)$,

$$
\begin{aligned}
B_{i}\left(u_{i, 0}, w\right) & :=\int_{\omega_{i}} a_{i} \nabla u_{i, 0} \cdot \nabla w \mathrm{~d} x \\
& =\int_{\omega_{i}} f w \mathrm{~d} x-\int_{\omega_{i}} a_{i} \nabla R_{g_{D}} \cdot \nabla w \mathrm{~d} x+\int_{\partial \omega_{i} \cap \Gamma_{N}} g_{N} w \mathrm{~d} s=: F_{i}(w) .
\end{aligned}
$$

The state solution $v_{i} \in H_{D}^{1}\left(\omega_{i}\right)$ verifies, for $i=1,2$,

$$
B_{i}\left(v_{i}, w\right)=0 \quad \forall w \in H_{D, \Gamma_{i}}^{1}\left(\omega_{i}\right) .
$$

Thanks to the Lax-Milgram lemma, the solutions $u_{1,0}^{\varepsilon}$ and $u_{2,0}^{0}$ exist and are unique. Moreover, if the virtual controls $\theta_{1}$ and $\theta_{2}$ are given, the solutions $v_{1}^{\varepsilon}$ and $v_{2}^{0}$ can be uniquely determined. The solutions $u_{1,0}^{\varepsilon}$ and $u_{2,0}^{0}$ can be computed before the coupling, as they are independent of the virtual controls $\left(\theta_{1}, \theta_{2}\right)$.

Homogenization method. As mentioned at the beginning of this section, the homogenization method can be used in $\omega_{2}$ to capture the effective behavior $u_{2}^{0}$. With additional information on the structure of the tensor $a_{2}^{\varepsilon}$, such as $a_{2}^{\varepsilon}(x)=a_{2}(x, x / \varepsilon)=$ $a_{2}(x, y)$ is $Y$-periodic in $y$, where $Y=(0,1)^{d}$, the homogenized tensor $a_{2}^{0}$ can be explicitly computed as

$$
a_{2}^{0}(x)=\frac{1}{|Y|} \int_{Y} a_{2}(x, y)(I+\nabla \chi) \mathrm{d} y,
$$

Copyright $@$ by SIAM. Unauthorized reproduction of this article is prohibited. 
where $\nabla \chi=\left(\nabla \chi^{1}, \ldots, \nabla \chi^{d}\right)$ and $I$ denotes the $d \times d$ identity matrix. Let $\left(e_{i}\right)_{i=1}^{d}$ be the canonical basis of $\mathbb{R}^{d}$. The functions $\chi^{j} \in W_{\mathrm{per}}^{1}(Y)$ are called the first order correctors, and, for $j=1, \ldots, d, \chi^{j}$ is the solution of the cell problem

$$
\int_{Y} a_{2}(x, y) \nabla \chi^{j} \cdot \nabla v \mathrm{~d} y=-\int_{Y} a_{2}(x, y) e_{j} \nabla v \mathrm{~d} y \quad \forall v \in W_{\mathrm{per}}^{1}(Y),
$$

with periodic boundary conditions.

This homogenized solution $u_{2}^{0}$ will be a good approximation of $u^{\varepsilon}$ in the $L^{2}$ norm but will fail in the $H^{1}$ norm. However, we can correct the homogenized solution and prove convergence in the $H^{1}$ norm in a subregion of $\omega_{2}$. Let $u^{0}$ be the homogenized solution corresponding to $u^{\varepsilon}$ in $\omega_{2}$ with, for all $x \in \Gamma_{2}, u^{0}(x)=u^{\varepsilon}(x)$ in the sense of the trace. Then, $u^{0}$ can be corrected using the periodic correctors $\chi^{j}$, and we obtain convergence to $u^{\varepsilon}$ in the $H^{1}$ norm on $\omega_{2}$,

$$
\left\|u^{\varepsilon}-\left(u^{0}+\varepsilon w(x, x / \varepsilon)\right)\right\|_{\mathrm{H}^{1}\left(\omega_{2}\right)} \leq C \varepsilon^{1 / 2},
$$

where the corrector term $w(x, x / \varepsilon)$ is given by

$$
w\left(x, \frac{x}{\varepsilon}\right)=\sum_{j=1}^{d} \chi^{j}\left(x, \frac{x}{\varepsilon}\right) \frac{\partial u^{0}(x)}{\partial x_{j}}, \quad x \in \omega_{2} .
$$

This will be explained in detail during the analysis in section 3. For classical results and proofs in homogenization, see, among others, [10, 28].

Nonoverlapping domain decomposition. The strategy relies on overlapping domain decomposition, but one could treat the problem using a domain decomposition method without overlapping domains [38]. Let $n$ stand for the outer normal derivative at the interface $\Gamma_{2}$. The problem will be as follows: find $u_{1}^{\varepsilon} \in H^{1}(\omega), u_{2}^{0} \in H^{1}\left(\omega_{2}\right)$ satisfying

$$
\begin{aligned}
-\operatorname{div}\left(a_{\omega}^{\varepsilon} \nabla u_{1}^{\varepsilon}\right) & =f & & \text { in } \omega, \\
u_{1}^{\varepsilon} & =u_{2}^{0} & & \text { on } \Gamma_{2}, \\
n \cdot\left(a_{\omega}^{\varepsilon} \nabla u_{1}^{\varepsilon}\right) & =n \cdot\left(a_{2}^{0} \nabla u_{2}^{0}\right) & & \text { on } \Gamma_{2}, \\
-\operatorname{div}\left(a_{2}^{0} \nabla u_{2}^{0}\right) & =f & & \text { in } \omega_{2},
\end{aligned}
$$

with the boundary conditions on $\Gamma_{D}$ and $\Gamma_{N}$ inherited from problem (2.1).

The Euler-Lagrange variational formulation. The minimization problem reads as follows: find $\left(\theta_{1}, \theta_{2}\right) \in \mathcal{U}$ such that

$$
J\left(\theta_{1}, \theta_{2}\right)=\min _{\left(\mu_{1}, \mu_{2}\right) \in \mathcal{U}} J\left(\mu_{1}, \mu_{2}\right)=\min _{\left(\mu_{1}, \mu_{2}\right) \in \mathcal{U}} \frac{1}{2}\left\|u_{1}^{\varepsilon}\left(\mu_{1}\right)-u_{2}^{0}\left(\mu_{2}\right)\right\|_{L^{2}\left(\omega_{0}\right)}^{2} .
$$

Using the splitting into $v_{i}\left(\mu_{i}\right)$ and $u_{i, 0}$, the cost $J$ can be written as

$$
\begin{aligned}
J\left(\mu_{1}, \mu_{2}\right)= & \frac{1}{2}\left\|v_{1}^{\varepsilon}\left(\mu_{1}\right)-v_{2}^{0}\left(\mu_{2}\right)\right\|_{\mathrm{L}^{2}\left(\omega_{0}\right)}^{2}+\frac{1}{2}\left\|u_{1,0}^{\varepsilon}-u_{2,0}^{0}\right\|_{\mathrm{L}^{2}\left(\omega_{0}\right)}^{2} \\
& +\int_{\omega_{0}}\left(v_{1}^{\varepsilon}\left(\mu_{1}\right)-v_{2}^{0}\left(\mu_{2}\right)\right)\left(u_{1,0}^{\varepsilon}-u_{2,0}^{0}\right) \mathrm{d} x \\
= & \frac{1}{2} \pi\left(\left(\mu_{1}, \mu_{2}\right),\left(\mu_{1}, \mu_{2}\right)\right)-F\left(\mu_{1}, \mu_{2}\right)+\frac{1}{2}\left\|u_{1,0}^{\varepsilon}-u_{2,0}^{0}\right\|_{\mathrm{L}^{2}\left(\omega_{0}\right)}^{2}
\end{aligned}
$$

Copyright $\odot$ by SIAM. Unauthorized reproduction of this article is prohibited. 
where $\pi: \mathcal{U} \times \mathcal{U} \rightarrow \mathbb{R}$ is given by

$$
\pi\left(\left(\theta_{1}, \theta_{2}\right),\left(\mu_{1}, \mu_{2}\right)\right)=\int_{\omega_{0}}\left(v_{1}^{\varepsilon}\left(\theta_{1}\right)-v_{2}^{0}\left(\theta_{2}\right)\right)\left(v_{1}^{\varepsilon}\left(\mu_{1}\right)-v_{2}^{0}\left(\mu_{2}\right)\right) \mathrm{d} x
$$

and $F: \mathcal{U} \rightarrow \mathbb{R}$ is given by

$$
F\left(\mu_{1}, \mu_{2}\right)=-\int_{\omega_{0}}\left(v_{1}^{\varepsilon}\left(\mu_{1}\right)-v_{2}^{0}\left(\mu_{2}\right)\right)\left(u_{1,0}^{\varepsilon}-u_{2,0}^{0}\right) \mathrm{d} x
$$

for $\left(\theta_{1}, \theta_{2}\right),\left(\mu_{1}, \mu_{2}\right) \in \mathcal{U}$. Following [29], the existence and uniqueness of the optimal controls hold when the form $\pi$ is a scalar product on the space of admissible controls. To prove the coercivity of the form $\pi$, we need a strong version of the Cauchy-Schwarz inequality. The proof is given in Appendix A.1.

Lemma A.3 (strong Cauchy-Schwarz). Let $v_{1}^{\varepsilon} \in H_{D}^{1}\left(\omega_{1}\right)$ and $v_{2}^{0} \in H_{D}^{1}\left(\omega_{2}\right)$ be solutions of (2.4) for $i=1,2$, respectively. Then, there exist an $\varepsilon_{0}>0$ and a positive constant $C_{s}<1$ such that for all $\varepsilon \leq \varepsilon_{0}$, it holds that

$$
\int_{\omega_{0}} v_{1}^{\varepsilon} v_{2}^{0} \mathrm{~d} x \leq C_{s}\left\|v_{1}^{\varepsilon}\right\|_{L^{2}\left(\omega_{0}\right)}\left\|v_{2}^{0}\right\|_{L^{2}\left(\omega_{0}\right)} .
$$

Lemma 2.1. Let $v_{1}^{\varepsilon}$ and $v_{2}^{0}$ be solutions of (2.4) for $i=1,2$, respectively. The following bounds hold:

$$
\begin{aligned}
\left\|v_{1}^{\varepsilon}\right\|_{\mathrm{L}^{2}(\omega)} & \leq \frac{C}{\tau}\left\|v_{1}^{\varepsilon}\right\|_{\mathrm{L}^{2}\left(\omega_{0}\right)}, \\
\left\|v_{2}^{0}\right\|_{\mathrm{L}^{2}\left(\Omega \backslash \omega_{1}\right)} & \leq \frac{C}{\tau}\left\|v_{2}^{0}\right\|_{\mathrm{L}^{2}\left(\omega_{0}\right)},
\end{aligned}
$$

where $\tau$ is the width of the overlap and $C$ is a constant depending on $\alpha, \beta$, and the Poincaré constant associated to $\omega_{1}$ and $\omega_{2}$, respectively.

Proof. We prove the lemma for the function $v_{1}^{\varepsilon}$. Let $\eta$ be a cutoff function such that $\eta=1$ in $\bar{\omega}, \eta=0$ in $\Omega \backslash \omega_{1}$, and $|\nabla \eta| \leq 1 / \tau$. Further, we have $\operatorname{supp}(\nabla \eta) \subset \omega_{0}$. Then $\eta v_{1}^{\varepsilon} \in H_{0}^{1}\left(\omega_{1}\right)$, and using the Poincaré inequality, it holds that

$$
\left\|v_{1}^{\varepsilon}\right\|_{\mathrm{L}^{2}(\omega)} \leq\left\|\eta v_{1}^{\varepsilon}\right\|_{\mathrm{L}^{2}\left(\omega_{1}\right)} \leq C_{\omega_{1}}\left\|\nabla\left(\eta v_{1}^{\varepsilon}\right)\right\|_{\mathrm{L}^{2}\left(\omega_{1}\right)} .
$$

The proof follows from the Caccioppoli inequality lemma, Lemma A.2, as

$$
\left\|\nabla\left(\eta v_{1}^{\varepsilon}\right)\right\|_{\mathrm{L}^{2}\left(\omega_{1}\right)}^{2} \leq \frac{\beta}{\alpha \tau^{2}}\left\|v_{1}^{\varepsilon}\right\|_{\mathrm{L}^{2}\left(\omega_{0}\right)}^{2}
$$

We obtain

$$
\left\|v_{1}^{\varepsilon}\right\|_{\mathrm{L}^{2}(\omega)} \leq C_{\omega_{1}} \sqrt{\frac{\beta}{\alpha}} \frac{1}{\tau}\left\|v_{1}^{\varepsilon}\right\|_{\mathrm{L}^{2}\left(\omega_{0}\right)}
$$

The proof is similar for $v_{2}^{0}$.

Lemma 2.2. Let $\varepsilon_{0}$ be given by the strong Cauchy-Schwarz lemma, Lemma A.3, and assume that $\varepsilon \leq \varepsilon_{0}$. Then, the form $\pi$ defines an inner product on $\mathcal{U}$.

Proof. The bilinearity, symmetry, and positivity are clear. We prove that the form is definite, i.e., $\pi\left(\left(\mu_{1}, \mu_{2}\right),\left(\mu_{1}, \mu_{2}\right)\right)=0$ if and only if $\left(\mu_{1}, \mu_{2}\right)=(0,0)$.

On the one hand, if the virtual controls are zero traces or fluxes, the state functions $v_{1}^{\varepsilon}$ and $v_{2}^{0}$ must be zero everywhere, as they are solutions of boundary value problems with zero right-hand side and boundary conditions. Thus $\pi\left(\left(\mu_{1}, \mu_{2}\right),\left(\mu_{1}, \mu_{2}\right)\right)=0$.

Copyright $@$ by SIAM. Unauthorized reproduction of this article is prohibited. 
On the other hand, using the strong Cauchy-Schwarz lemma, Lemma A.3,

$$
\begin{aligned}
0 & =\pi\left(\left(\mu_{1}, \mu_{2}\right),\left(\mu_{1}, \mu_{2}\right)\right)=\left\|v_{1}^{\varepsilon}\left(\mu_{1}\right)-v_{2}^{0}\left(\mu_{2}\right)\right\|_{\mathrm{L}^{2}\left(\omega_{0}\right)}^{2} \\
& =\left\|v_{1}^{\varepsilon}\left(\mu_{1}\right)\right\|_{\mathrm{L}^{2}\left(\omega_{0}\right)}^{2}+\left\|v_{2}^{0}\left(\mu_{2}\right)\right\|_{\mathrm{L}^{2}\left(\omega_{0}\right)}^{2}-2 \int_{\omega_{0}} v_{1}^{\varepsilon}\left(\mu_{1}\right) v_{2}^{0}\left(\mu_{2}\right) \mathrm{d} x \\
& \geq\left\|v_{1}^{\varepsilon}\left(\mu_{1}\right)\right\|_{\mathrm{L}^{2}\left(\omega_{0}\right)}^{2}+\left\|v_{2}^{0}\left(\mu_{2}\right)\right\|_{\mathrm{L}^{2}\left(\omega_{0}\right)}^{2}-2 C_{s}\left\|v_{1}^{\varepsilon}\left(\mu_{1}\right)\right\|_{\mathrm{L}^{2}\left(\omega_{0}\right)}\left\|v_{2}^{0}\left(\mu_{2}\right)\right\|_{\mathrm{L}^{2}\left(\omega_{0}\right)} \\
& \geq\left(1-C_{s}\right)\left(\left\|v_{1}^{\varepsilon}\left(\mu_{1}\right)\right\|_{\mathrm{L}^{2}\left(\omega_{0}\right)}^{2}+\left\|v_{2}^{0}\left(\mu_{2}\right)\right\|_{\mathrm{L}^{2}\left(\omega_{0}\right)}^{2}\right) .
\end{aligned}
$$

As $C_{s}<1$, it holds that $\left\|v_{1}^{\varepsilon}\left(\mu_{1}\right)\right\|_{\mathrm{L}^{2}\left(\omega_{0}\right)}=\left\|v_{2}^{0}\left(\mu_{2}\right)\right\|_{\mathrm{L}^{2}\left(\omega_{0}\right)}=0$, which implies that $v_{1}^{\varepsilon}=v_{2}^{0}=0$, a.e. in $\omega_{0}$. By Lemma 2.1, we then have that $\left\|v_{1}^{\varepsilon}\left(\mu_{1}\right)\right\|_{\mathrm{L}^{2}(\omega)}=0$ and $\left\|v_{2}^{0}\left(\mu_{2}\right)\right\|_{\mathrm{L}^{2}\left(\Omega \backslash \omega_{1}\right)}=0$, and thus $v_{i}=0$ a.e. in $\omega_{i}$ for $i=1,2$. Then we obtain, for $i=1,2$,

$$
\left\|\mu_{i}\right\|_{\mathrm{H}^{1 / 2}\left(\Gamma_{i}\right)} \leq C_{i}\left\|v_{i}\left(\mu_{i}\right)\right\|_{\mathrm{H}^{1}\left(\omega_{i}\right)}=0
$$

where the constants depend on $\omega_{i}$, and the trace operators $\gamma_{i}: H^{1 / 2}\left(\Gamma_{i}\right) \rightarrow H^{1}\left(\omega_{i}\right)$. Thus, $\mu_{i}=0$ on $\Gamma_{i}$, and the form $\pi$ is an inner product on $\mathcal{U}$.

We can then define a norm on $\mathcal{U}$ induced by the inner product $\pi$. For a pair $\left(\mu_{1}, \mu_{2}\right) \in \mathcal{U}$, we set

$$
\left\|\left(\mu_{1}, \mu_{2}\right)\right\|_{\mathrm{L}^{\star}(\mathcal{U})}:=\left\|v_{1}^{\varepsilon}\left(\mu_{1}\right)-v_{2}^{0}\left(\mu_{2}\right)\right\|_{\mathrm{L}^{2}\left(\omega_{0}\right)} .
$$

The space $\mathcal{U}$ might not be complete with respect to this norm, but we can construct a completion of $\mathcal{U}$ and solve the minimization problem in the completed space. Let us denote the completed control space by $\hat{\mathcal{U}}$. Using the Hahn-Banach theorem, the inner product $\pi$ and the functional $F$ can be continuously extended in a unique way on $\hat{\mathcal{U}}$, and we denote these extensions by $\hat{\pi}$ and $\hat{F}$. The form $\hat{\pi}$ is continuous, symmetric, and coercive in $\hat{\mathcal{U}}$. The existence and uniqueness of the optimal pair in $\hat{\mathcal{U}}$ is given in the next theorem.

THEOREM 2.3. The minimization problem (2.7) has a unique solution $\left(\theta_{1}, \theta_{2}\right) \in$ $\hat{U}$ that satisfies the Euler-Lagrange equation

$$
\hat{\pi}\left(\left(\theta_{1}, \theta_{2}\right),\left(\mu_{1}, \mu_{2}\right)\right)=\hat{F}\left(\mu_{1}, \mu_{2}\right) \quad \forall\left(\mu_{1}, \mu_{2}\right) \in \hat{U},
$$

where $\hat{\pi}$ and $\hat{F}$ are the continuous extensions of $\pi$ and $F$ given by (2.8) and (2.9).

Proof. The existence and uniqueness of $\left(\theta_{1}, \theta_{2}\right) \in \hat{\mathcal{U}}$ follows from [29, Theorem I. 1.1], as the form $\hat{\pi}$ is symmetric, continuous, and coercive, and $\hat{F}$ is continuous.

The optimal pair $\left(\theta_{1}, \theta_{2}\right) \in \hat{\mathcal{U}}$ minimizes the cost function, but in general there exist no functions $u_{i} \in H^{1}\left(\omega_{i}\right)$ that satisfy (2.3). However, there exists an embedding $\sigma: \mathcal{U} \rightarrow \hat{\mathcal{U}}$ such that $\sigma(\mathcal{U})$ is dense in $\hat{\mathcal{U}}$. Further, we can identify $\mathcal{U}$ with $\sigma(\mathcal{U})$ and conclude that $\left(\theta_{1}, \theta_{2}\right)$ is the limit of a sequence $\left(\theta_{1_{n}}, \theta_{2_{n}}\right)_{n \in \mathbb{N}}$ with $u_{i}\left(\theta_{i_{n}}\right) \in H^{1}\left(\omega_{i}\right)$ satisfying (2.3). In what follows, for simplicity, we assume that the optimal pair is in $\mathcal{U}$, and hence $u_{i}\left(\theta_{i}\right) \in H^{1}\left(\omega_{i}\right)$ for $i=1,2$ (we then also have $v_{i}\left(\theta_{i}\right) \in H^{1}\left(\omega_{i}\right)$ ).

Optimality system. The state solutions and the optimal controls $\left(\theta_{1}, \theta_{2}\right) \in \mathcal{U}$ are obtained by solving an optimality system, derived from the minimization problem. The boundary value problems on $\omega_{1}$ and $\omega_{2}$ act as constraints. Let $\lambda_{i}, i=1,2$, be Lagrange multipliers associated to the constraints in $\omega_{i}$, and consider the critical 
point of the Lagrangian functional

$$
\begin{aligned}
\mathcal{L}\left(u_{1}^{\varepsilon}, \lambda_{1}, \theta_{1}, u_{2}^{0}, \lambda_{2}, \theta_{2}\right)= & \frac{1}{2}\left\|u_{1}^{\varepsilon}-u_{2}^{0}\right\|_{L^{2}\left(\omega_{0}\right)}^{2}+\left\langle f+\operatorname{div}\left(a_{1}^{\varepsilon} \nabla u_{1}^{\varepsilon}\right), \lambda_{1}\right\rangle_{H^{-1}, H^{1}} \\
& +\left\langle f+\operatorname{div}\left(a_{2}^{0} \nabla u_{2}^{0}\right), \lambda_{2}\right\rangle_{H^{-1}, H^{1}},
\end{aligned}
$$

with $u_{i} \in H_{D}^{1}\left(\omega_{i}\right)$ and $\lambda_{i} \in H^{2}\left(\omega_{i}\right)$, with $\lambda_{i}=0$ on $\partial \omega_{i} \cap \Gamma_{D}$ and $\Gamma_{i}$, and $n_{i} \cdot\left(a_{i} \nabla \lambda_{i}\right)=$ 0 on $\partial \omega_{i} \cap \Gamma_{N}$ for $i=1,2$. Using the transposition method [30], we can write the right-hand side of the Lagrangian in terms of the state, Lagrange multipliers, and control variables. Computing the Gâteaux derivatives for each of the unknowns leads to the optimality system.

We note that the optimality system can also be derived by using the adjoint problems of (2.3).

3. A priori error analysis of the continuous coupled problem. In this section, we give an a priori error analysis of the optimization based method. The analysis is separated into fine and coarse scale error estimates. The solution of the minimization problem with constraints (2.3) gives us a fine scale solution in $\omega_{1}$ and a coarse scale solution in $\omega_{2}$. Looking at the error between the solution of the coupling and the exact fine scale solution $u^{\varepsilon}$ on either $\omega_{1}$ or $\omega_{2}$ obliges us to estimate terms on the boundary $\Gamma_{1}$ or $\Gamma_{2}$, respectively. In order to avoid such additional error terms, we introduce an intermediate domain $\omega^{+}$with $\omega \subset \omega^{+} \subset \omega_{1}$. Then given $u_{1}^{\varepsilon}\left(\theta_{1}\right)$ and $u_{2}^{0}\left(\theta_{2}\right)$, the solutions of the optimization based coupling method, we define

$$
\bar{u}^{\varepsilon}= \begin{cases}u_{1}^{\varepsilon}\left(\theta_{1}\right) & \text { in } \omega^{+} \\ u_{2}^{r e c}\left(\theta_{2}\right) & \text { in } \Omega \backslash \omega^{+}\end{cases}
$$

where $u_{2}^{r e c}$ stands for a correction to the homogenized solution $u_{2}^{0}\left(\theta_{2}\right)$ given below. The main convergence results are

$$
\begin{aligned}
\left\|u^{\varepsilon}-\bar{u}^{\varepsilon}\right\|_{\mathrm{H}^{1}\left(\omega^{+}\right)} & \leq C \varepsilon, \\
\left\|u^{\varepsilon}-\bar{u}^{\varepsilon}\right\|_{\mathrm{H}^{1}\left(\Omega \backslash \omega^{+}\right)} & \leq C \varepsilon^{1 / 2},
\end{aligned}
$$

where the constants depend on the width of $\omega^{+}$and the ellipticity constants of $a^{\varepsilon}$. For the analysis, we consider the classical locally periodic correctors $\chi^{j}$ solutions of (2.6), but other postprocessing procedures could be used. The correction $u_{2}^{r e c}\left(\theta_{2}\right)$ is given by

$$
u_{2}^{r e c}(x)=u_{2}^{0}(x)+\varepsilon \sum_{j=1}^{d} \chi^{j}\left(x, \frac{x}{\varepsilon}\right) \frac{\partial u_{2}^{0}(x)}{\partial x_{j}}, \quad x \in \Omega \backslash \omega^{+},
$$

where $u_{2}^{0}=u_{2}^{0}\left(\theta_{2}\right)$. We sometimes use $u_{2}^{0}\left(\theta_{2}\right)$ and $u_{2}^{r e c}\left(\theta_{2}\right)$ to emphasize the dependency on $\theta_{2}$. We will, however, avoid the heavy notation $u_{2}^{0}\left(\theta_{2}\right)(x)$ and drop the dependency on $\theta_{2}$ when writing such maps as functions of $x$.

A priori error estimates for the fine scale solver in $\omega^{+}$. The coupled solution restricted to the subregion $\omega^{+}$is given by the fine scale solution $u_{1}^{\varepsilon}\left(\theta_{1}\right)$; hence the error becomes $\left\|u^{\varepsilon}-\bar{u}^{\varepsilon}\right\|_{\mathrm{H}^{1}\left(\omega^{+}\right)}=\left\|u^{\varepsilon}-u_{1}^{\varepsilon}\left(\theta_{1}\right)\right\|_{\mathrm{H}^{1}\left(\omega^{+}\right)}$.

Let $\tau$ denote the width of the overlap $\omega_{0}$, and recall that the heterogeneous tensor $a_{2}^{\varepsilon}$ satisfies the ellipticity condition (2.2). Further, we denote by $\tau^{+}$the distance between $\partial \omega^{+}$and $\omega$; it holds that $\tau^{+}<\tau$. Moreover, we suppose that there exists $\varepsilon_{0}>0$ such that the strong Cauchy-Schwarz lemma, Lemma A.3, holds for all $\varepsilon \leq \varepsilon_{0}$. 
Let $\gamma_{i}: H^{1}\left(\omega_{i}\right) \rightarrow H^{1 / 2}\left(\Gamma_{i}\right), i=1,2$, be trace operators, and consider the solution $u^{\varepsilon}$ restricted to the domain $\omega_{2}$,

$$
\begin{aligned}
-\operatorname{div}\left(a_{2}^{\varepsilon} \nabla u^{\varepsilon}\right) & =f & & \text { in } \omega_{2}, \\
u^{\varepsilon} & =\gamma_{2}\left(u^{\varepsilon}\right) & & \text { on } \Gamma_{2}, \\
u^{\varepsilon} & =g_{D} & & \text { on } \partial \omega_{2} \cap \Gamma_{D}, \\
n_{2} \cdot\left(a_{2}^{\varepsilon} \nabla u^{\varepsilon}\right) & =g_{N} & & \text { on } \partial \omega_{2} \cap \Gamma_{N} .
\end{aligned}
$$

Further, for a fixed $\varepsilon \leq \varepsilon_{0}$, we introduce $u^{0} \in H^{1}\left(\omega_{2}\right)$, the homogenized solution of

$$
\begin{aligned}
-\operatorname{div}\left(a_{2}^{0} \nabla u^{0}\right) & =f & & \text { in } \omega_{2}, \\
u^{0} & =\gamma_{2}\left(u^{\varepsilon}\right) & & \text { on } \Gamma_{2}, \\
u^{0} & =g_{D} & & \text { on } \partial \omega_{2} \cap \Gamma_{D}, \\
n_{2} \cdot\left(a_{2}^{0} \nabla u^{0}\right) & =g_{N} & & \text { on } \partial \omega_{2} \cap \Gamma_{N} .
\end{aligned}
$$

We assume that strong convergence in the $L^{2}$ norm is available [28, sect. 1.4], i.e.,

$$
\left\|u^{\varepsilon}-u^{0}\right\|_{L^{2}\left(\omega_{2}\right)} \leq C \varepsilon .
$$

Remark 3.1. The error estimate (3.4) holds if $a_{2}(\cdot, y) \in W^{1, \infty}(Y)$ and $u^{0} \in$ $H^{2}\left(\omega_{2}\right)$. This can be seen by following the lines of the proof in [28]. Thanks to the regularity of $a_{2}^{\varepsilon}$, we have $\chi^{j} \in W^{1, \infty}(Y)$. The regularity on the tensor can be relaxed to $a_{2}(\cdot, y) \in W^{1, p}(Y)$ for $p>2$, and $\chi^{j} \in W^{1, p}(Y) \cap \mathcal{C}^{1, s}(\bar{Y})$ for $s=1-d / p$. For the proof of (3.4), we refer the reader to [28, 33].

We follow the framework introduced in [37] and define an operator $P: \mathcal{U} \rightarrow$ $H^{1}\left(\omega_{1}\right) \times H^{1}\left(\Omega \backslash \omega_{1}\right)$ by

$$
\left(\mu_{1}, \mu_{2}\right) \mapsto P\left(\mu_{1}, \mu_{2}\right)= \begin{cases}u_{1,0}^{\varepsilon}+v_{1}^{\varepsilon}\left(\mu_{1}\right) & \text { in } \omega_{1}, \\ u_{2,0}^{0}+v_{2}^{0}\left(\mu_{2}\right) & \text { in } \Omega \backslash \omega_{1},\end{cases}
$$

where $v_{i}$ are solutions of (2.4) for $i=1,2$. We note that for the traces $\left(\gamma_{1}\left(u^{\varepsilon}\right), \gamma_{2}\left(u^{\varepsilon}\right)\right)$ of the exact solution $u^{\varepsilon}$, we obtain

$$
P\left(\gamma_{1}\left(u^{\varepsilon}\right), \gamma_{2}\left(u^{\varepsilon}\right)\right)= \begin{cases}u^{\varepsilon} & \text { in } \omega_{1}, \\ u^{0} & \text { in } \Omega \backslash \omega_{1} .\end{cases}
$$

The operator $P$ can be split into $P\left(\mu_{1}, \mu_{2}\right)=U_{0}+Q\left(\mu_{1}, \mu_{2}\right)$ for $\left(\mu_{1}, \mu_{2}\right) \in \mathcal{U}$, where we define

$$
U_{0}=\left\{\begin{array}{ll}
u_{1,0}^{\varepsilon} & \text { in } \omega_{1}, \\
u_{2,0}^{0} & \text { in } \Omega \backslash \omega_{1}
\end{array} \quad \text { and } \quad Q\left(\mu_{1}, \mu_{2}\right)= \begin{cases}v_{1}^{\varepsilon}\left(\mu_{1}\right) & \text { in } \omega_{1} \\
v_{2}^{0}\left(\mu_{2}\right) & \text { in } \Omega \backslash \omega_{1}\end{cases}\right.
$$

TheOREM 3.2. Let $u^{\varepsilon}$ be the solution of (2.1), and let $\bar{u}^{\varepsilon}$ be given by (3.1). Suppose that $u^{0}$ and $\chi^{j}$ are regular enough so that (3.4) holds. Let $\varepsilon_{0}$ be given by the strong Cauchy-Schwarz lemma, Lemma A.3, and assume that $\varepsilon \leq \varepsilon_{0}$. Then, we have

$$
\left\|u^{\varepsilon}-u_{1}^{\varepsilon}\left(\theta_{1}\right)\right\|_{\mathrm{H}^{1}\left(\omega^{+}\right)} \leq C \varepsilon,
$$

where the constant $C$ depends on $\tau, \tau^{+}, \alpha, \beta$, and on the domains $\omega_{1}$ and $\omega_{2}$.

Copyright $@$ ㅇ by SIAM. Unauthorized reproduction of this article is prohibited. 
Proof. The difference $u^{\varepsilon}-u_{1}^{\varepsilon}\left(\theta_{1}\right) \in H_{D}^{1}\left(\omega_{1}\right)$ is $a^{\varepsilon}$-harmonic in $\omega_{1}$, and the Caccioppoli inequality theorem, Theorem A.1, can be applied; that is,

$$
\left\|u^{\varepsilon}-u_{1}^{\varepsilon}\left(\theta_{1}\right)\right\|_{\mathrm{H}^{1}\left(\omega^{+}\right)} \leq \frac{C}{\tau-\tau^{+}}\left\|u^{\varepsilon}-u_{1}^{\varepsilon}\left(\theta_{1}\right)\right\|_{\mathrm{L}^{2}\left(\omega_{1}\right)},
$$

where the constant $C$ depends on the ellipticity constants of the tensor $a^{\varepsilon}$. Let us focus on the $L^{2}$ norm; recalling that $u_{1}^{\varepsilon}\left(\theta_{1}\right)=P\left(\theta_{1}, \theta_{2}\right)$, it holds that

$$
\begin{aligned}
& \left\|u^{\varepsilon}-u_{1}^{\varepsilon}\left(\theta_{1}\right)\right\|_{\mathrm{L}^{2}\left(\omega_{1}\right)}=\left\|u^{\varepsilon}-P\left(\theta_{1}, \theta_{2}\right)\right\|_{\mathrm{L}^{2}\left(\omega_{1}\right)} \\
& \leq\left\|u^{\varepsilon}-P\left(\gamma_{1}\left(u^{\varepsilon}\right), \gamma_{2}\left(u^{\varepsilon}\right)\right)\right\|_{\mathrm{L}^{2}\left(\omega_{1}\right)}+\left\|P\left(\gamma_{1}\left(u^{\varepsilon}\right), \gamma_{2}\left(u^{\varepsilon}\right)\right)-P\left(\theta_{1}, \theta_{2}\right)\right\|_{\mathrm{L}^{2}\left(\omega_{1}\right)} .
\end{aligned}
$$

By the definitions of $P$ and $u^{\varepsilon}$, the first $L^{2}$ error is zero, and it remains to bound the second $L^{2}$ error

$$
\begin{aligned}
\left\|u^{\varepsilon}-u_{1}^{\varepsilon}\left(\theta_{1}\right)\right\|_{\mathrm{L}^{2}\left(\omega_{1}\right)} & \leq\left\|P\left(\gamma_{1}\left(u^{\varepsilon}\right), \gamma_{2}\left(u^{\varepsilon}\right)\right)-P\left(\theta_{1}, \theta_{2}\right)\right\|_{\mathrm{L}^{2}\left(\omega_{1}\right)} \\
& =\left\|U_{0}-Q\left(\gamma_{1}\left(u^{\varepsilon}\right), \gamma_{2}\left(u^{\varepsilon}\right)\right)-U_{0}+Q\left(\theta_{1}, \theta_{2}\right)\right\|_{\mathrm{L}^{2}\left(\omega_{1}\right)} \\
& \leq\|Q\|\left\|\left(\gamma_{1}\left(u^{\varepsilon}\right), \gamma_{2}\left(u^{\varepsilon}\right)\right)-\left(\theta_{1}, \theta_{2}\right)\right\|_{\mathrm{L}^{*}(\mathcal{U})},
\end{aligned}
$$

where the norm $\|\cdot\|_{\mathrm{L}^{*}(\mathcal{U})}$ is induced by the inner product $\pi$ and defined in (2.10). Using Lemmas 3.3 and 3.4 given below proves the result.

Lemma 3.3. Let $u^{\varepsilon}$ and $u^{0}$ solve (2.1) and (3.3), respectively, and let $\left(\theta_{1}, \theta_{2}\right) \in \mathcal{U}$ be the optimal virtual controls. Then

$$
\left\|\left(\gamma_{1}\left(u^{\varepsilon}\right), \gamma_{2}\left(u^{\varepsilon}\right)\right)-\left(\theta_{1}, \theta_{2}\right)\right\|_{L^{*}(\mathcal{U})} \leq\left\|u^{\varepsilon}-u^{0}\right\|_{L^{2}\left(\omega_{0}\right)} .
$$

Proof. By definition, we have

$$
\begin{aligned}
& \left\|\left(\gamma_{1}\left(u^{\varepsilon}\right), \gamma_{2}\left(u^{\varepsilon}\right)\right)-\left(\theta_{1}, \theta_{2}\right)\right\|_{L^{*}(\mathcal{U})} \\
& =\sup _{\left(\mu_{1}, \mu_{2}\right) \in \mathcal{U}} \frac{\left|\pi\left(\left(\gamma_{1}\left(u^{\varepsilon}\right), \gamma_{2}\left(u^{\varepsilon}\right)\right),\left(\mu_{1}, \mu_{2}\right)\right)-\pi\left(\left(\theta_{1}, \theta_{2}\right),\left(\mu_{1}, \mu_{2}\right)\right)\right|}{\left\|\left(\mu_{1}, \mu_{2}\right)\right\|_{\mathrm{L}^{*}(\mathcal{U})}} .
\end{aligned}
$$

We look at the numerator. As the pair $\left(\theta_{1}, \theta_{2}\right)$ minimizes the cost function $J$, the Euler-Lagrange formulation (2.11) holds, and

$$
\begin{aligned}
\pi & \left(\left(\gamma_{1}\left(u^{\varepsilon}\right), \gamma_{2}\left(u^{\varepsilon}\right)\right),\left(\mu_{1}, \mu_{2}\right)\right)-\pi\left(\left(\theta_{1}, \theta_{2}\right),\left(\mu_{1}, \mu_{2}\right)\right) \\
= & \int_{\omega_{0}}\left(v_{1}^{\varepsilon}\left(\gamma_{1}\left(u^{\varepsilon}\right)\right)-v_{2}^{0}\left(\gamma_{2}\left(u^{\varepsilon}\right)\right)\right)\left(v_{1}^{\varepsilon}\left(\mu_{1}\right)-v_{2}^{0}\left(\mu_{2}\right)\right) \mathrm{d} x \\
& +\int_{\omega_{0}}\left(v_{1}^{\varepsilon}\left(\mu_{1}\right)-v_{2}^{0}\left(\mu_{2}\right)\right)\left(u_{1,0}^{\varepsilon}-u_{2,0}^{0}\right) \mathrm{d} x \\
= & \int_{\omega_{0}}\left(\left(v_{1}^{\varepsilon}\left(\gamma_{1}\left(u^{\varepsilon}\right)\right)+u_{1,0}^{\varepsilon}\right)-\left(v_{2}^{0}\left(\gamma_{2}\left(u^{\varepsilon}\right)\right)+u_{2,0}^{0}\right)\right)\left(v_{1}^{\varepsilon}\left(\mu_{1}\right)-v_{2}^{0}\left(\mu_{2}\right)\right) \mathrm{d} x \\
= & \int_{\omega_{0}}\left(u^{\varepsilon}-u^{0}\right)\left(v_{1}^{\varepsilon}\left(\mu_{1}\right)-v_{2}^{0}\left(\mu_{2}\right)\right) \mathrm{d} x \leq\left\|u^{\varepsilon}-u^{0}\right\|_{\mathrm{L}^{2}\left(\omega_{0}\right)}\left\|\left(\mu_{1}, \mu_{2}\right)\right\|_{\mathrm{L}^{*}(\mathcal{U})} .
\end{aligned}
$$

The result follows.

To complete the a priori error analysis in the continuous case, we need to bound the norm of the operator $Q$.

Copyright (c) by SIAM. Unauthorized reproduction of this article is prohibited. 
Lemma 3.4. Let $\varepsilon_{0}$ be given by the strong Cauchy-Schwarz lemma, Lemma A.3, and assume that $\varepsilon \leq \varepsilon_{0}$. The operator $Q$, defined by (3.6), is bounded from $L^{2}(\Omega)$ to $L^{*}(\mathcal{U})$ :

$$
\|Q\| \leq C
$$

where the constant $C$ depends on $\omega_{1}, \omega_{2}, \tau$, and the strong Cauchy-Schwarz constant; see Lemma A.3.

Proof. By definition, the norm of the operator $Q$ is given by

$$
\|Q\|:=\sup _{\left(\mu_{1}, \mu_{2}\right) \in \mathcal{U}} \frac{\left\|Q\left(\mu_{1}, \mu_{2}\right)\right\|_{\mathrm{L}^{2}(\Omega)}}{\left\|\left(\mu_{1}, \mu_{2}\right)\right\|_{\mathrm{L}^{*}(\mathcal{U})}} .
$$

For $\left(\mu_{1}, \mu_{2}\right) \in \mathcal{U}$, we show the existence of a positive constant such that

$$
\left\|Q\left(\mu_{1}, \mu_{2}\right)\right\|_{\mathrm{L}^{2}(\Omega)}^{2} \leq C\left\|\left(\mu_{1}, \mu_{2}\right)\right\|_{\mathrm{L}^{*}(\mathcal{U})}^{2} .
$$

For simplicity, we set $v_{i}=v_{i}\left(\mu_{i}\right), i=1,2$. Using Lemma 2.1, we have

$$
\begin{aligned}
\left\|Q\left(\mu_{1}, \mu_{2}\right)\right\|_{\mathrm{L}^{2}(\Omega)}^{2} & =\left\|v_{1}^{\varepsilon}\right\|_{\mathrm{L}^{2}\left(\omega_{1}\right)}^{2}+\left\|v_{2}^{0}\right\|_{\mathrm{L}^{2}\left(\Omega \backslash \omega_{1}\right)}^{2} \\
& \leq \frac{C\left(\omega_{1} ; \omega_{2}\right)}{\tau^{2}}\left(\left\|v_{1}^{\varepsilon}\right\|_{\mathrm{L}^{2}\left(\omega_{0}\right)}^{2}+\left\|v_{2}^{0}\right\|_{\mathrm{L}^{2}\left(\omega_{0}\right)}^{2}\right) .
\end{aligned}
$$

Next, using the strong Cauchy-Schwarz lemma, Lemma A.3, yields

$$
\begin{aligned}
\left\|\left(\mu_{1}, \mu_{2}\right)\right\|_{\mathrm{L}^{*}(\mathcal{U})}^{2} & =\left\|v_{1}^{\varepsilon}-v_{2}^{0}\right\|_{\mathrm{L}^{2}\left(\omega_{0}\right)}^{2}=\left\|v_{1}^{\varepsilon}\right\|_{\mathrm{L}^{2}\left(\omega_{0}\right)}^{2}+\left\|v_{2}^{0}\right\|_{\mathrm{L}^{2}\left(\omega_{0}\right)}^{2}-2 \int_{\omega_{0}} v_{1}^{\varepsilon} v_{2}^{0} \mathrm{~d} x \\
& \geq\left\|v_{1}^{\varepsilon}\right\|_{\mathrm{L}^{2}\left(\omega_{0}\right)}^{2}+\left\|v_{2}^{0}\right\|_{\mathrm{L}^{2}\left(\omega_{0}\right)}^{2}-2 C_{s}\left\|v_{1}^{\varepsilon}\right\|_{\mathrm{L}^{2}\left(\omega_{0}\right)}\left\|v_{2}^{0}\right\|_{\mathrm{L}^{2}\left(\omega_{0}\right)} \\
& \geq\left(1-C_{s}\right)\left(\left\|v_{1}^{\varepsilon}\right\|_{\mathrm{L}^{2}\left(\omega_{0}\right)}^{2}+\left\|v_{2}^{0}\right\|_{\mathrm{L}^{2}\left(\omega_{0}\right)}^{2}\right) .
\end{aligned}
$$

Summarizing, this gives

$$
\left\|Q\left(\mu_{1}, \mu_{2}\right)\right\|_{\mathrm{L}^{2}(\Omega)}^{2} \leq \frac{C\left(\omega_{1} ; \omega_{2}\right)}{\tau^{2}\left(1-C_{s}\right)}\left\|\left(\mu_{1}, \mu_{2}\right)\right\|_{\mathrm{L}^{*}(\mathcal{U})}^{2} .
$$

A priori error estimates for the reconstructed coarse scale solver in $\Omega \backslash \omega^{+}$. In this section, we give an a priori error estimate in the coarse scale region $\Omega \backslash \omega^{+}$. The coupled solution restricted to the subregion $\Omega \backslash \omega^{+}$is given by $u_{2}^{r e c}\left(\theta_{2}\right)$.

Lemma 3.5. Let $u^{\varepsilon}$ and $u_{2}^{0}$ be the solutions of problems (2.1) and (2.3), respectively. Assuming that (3.4) holds, we obtain

$$
\left\|u^{\varepsilon}-u_{2}^{0}\left(\theta_{2}\right)\right\|_{L^{2}\left(\omega_{2}\right)} \leq C \varepsilon .
$$

Proof. We define an operator $P: \mathcal{U} \rightarrow H^{1}(\omega) \times H^{1}\left(\omega_{2}\right)$ by

$$
P\left(\mu_{1}, \mu_{2}\right)= \begin{cases}u_{1,0}^{\varepsilon}+v_{1}^{\varepsilon}\left(\mu_{1}\right) & \text { in } \omega, \\ u_{2,0}^{0}+v_{2}^{0}\left(\mu_{2}\right) & \text { in } \omega_{2},\end{cases}
$$

and consider the decomposition $P=U_{0}+Q$, following (3.6). It holds that $u_{2}^{0}\left(\theta_{2}\right)=$ $\left.P\left(\theta_{1}, \theta_{2}\right)\right|_{\omega_{2}}$, and

$$
\begin{aligned}
\left\|u^{\varepsilon}-u_{2}^{0}\left(\theta_{2}\right)\right\|_{\mathrm{L}^{2}\left(\omega_{2}\right)} \leq & \left\|u^{\varepsilon}-P\left(\gamma_{1}\left(u^{\varepsilon}\right), \gamma_{2}\left(u^{\varepsilon}\right)\right)\right\|_{\mathrm{L}^{2}\left(\omega_{2}\right)} \\
& +\left\|P\left(\gamma_{1}\left(u^{\varepsilon}\right), \gamma_{2}\left(u^{\varepsilon}\right)\right)-P\left(\theta_{1}, \theta_{2}\right)\right\|_{\mathrm{L}^{2}\left(\omega_{2}\right)} .
\end{aligned}
$$

Copyright (c) by SIAM. Unauthorized reproduction of this article is prohibited. 
The term $P\left(\gamma_{1}\left(u^{\varepsilon}\right), \gamma_{2}\left(u^{\varepsilon}\right)\right)$, restricted to $\omega_{2}$, is equal to $u_{2}^{0}\left(\gamma_{2}\left(u^{\varepsilon}\right)\right)$, which is defined as the homogenized solution $u^{0}$ obtained in (3.3). Using (3.4), we have

$$
\begin{aligned}
\left\|u^{\varepsilon}-u_{2}^{0}\left(\theta_{2}\right)\right\|_{L^{2}\left(\omega_{2}\right)} & \leq\left\|u^{\varepsilon}-u^{0}\right\|_{L^{2}\left(\omega_{2}\right)}+\left\|P\left(\gamma_{1}\left(u^{\varepsilon}\right), \gamma_{2}\left(u^{\varepsilon}\right)\right)-P\left(\theta_{1}, \theta_{2}\right)\right\|_{L^{2}\left(\omega_{2}\right)} \\
& \leq C \varepsilon+\|Q\|\left\|\left(\gamma_{1}\left(u^{\varepsilon}\right), \gamma_{2}\left(u^{\varepsilon}\right)\right)-\left(\theta_{1}, \theta_{2}\right)\right\|_{L^{*}(\mathcal{U})} .
\end{aligned}
$$

Following the proof of Lemma 3.4, we can show that $\|Q\|$ is bounded, and using Lemma 3.3, we obtain

$$
\left\|u^{\varepsilon}-u_{2}^{0}\left(\theta_{2}\right)\right\|_{\mathrm{L}^{2}\left(\omega_{2}\right)} \leq C_{1} \varepsilon+C_{2}\left\|u^{\varepsilon}-u^{0}\right\|_{\mathrm{L}^{2}\left(\omega_{0}\right)} \leq C \varepsilon .
$$

THEOREM 3.6. Let $u^{\varepsilon}$ be the solution of (2.1), and let $u_{2}^{\text {rec }}\left(\theta_{2}\right)$ be given by (3.2). Let $a_{2}(x, y) \in \mathcal{C}\left(\bar{\omega}_{2} ; L_{p e r}^{\infty}(Y)\right)$ and $\chi^{j} \in W_{p e r}^{1}(Y), j=1, \ldots, d$. If, in addition, $u^{\varepsilon} \in$ $H^{2}(\Omega), u_{2}^{0}\left(\theta_{2}\right) \in H^{2}\left(\omega_{2}\right)$, and $\chi^{j} \in W^{1, \infty}(Y), j=1, \ldots, d$, it holds that

$$
\left\|u^{\varepsilon}-u_{2}^{r e c}\left(\theta_{2}\right)\right\|_{\mathrm{H}^{1}\left(\Omega \backslash \omega^{+}\right)} \leq C \varepsilon^{1 / 2},
$$

where the constant $C$ is independent of $\varepsilon$, but depends on $\tau, \tau^{+}$, and the ellipticity constants of $a_{2}^{\varepsilon}$.

Proof. Recall that $u^{0}$ is the homogenized solution of (3.3); using the periodic corrector $\chi$, we have a reconstructed solution $u^{0, r e c}$ given by

$$
u^{0, r e c}(x)=u^{0}(x)+\varepsilon \sum_{j=1}^{d} \chi^{j}\left(x, \frac{x}{\varepsilon}\right) \frac{\partial u^{0}(x)}{\partial x_{j}} .
$$

Using the triangular inequality with $u^{0, r e c}$, we have

$$
\left\|u^{\varepsilon}-u_{2}^{r e c}\left(\theta_{2}\right)\right\|_{\mathrm{H}^{1}\left(\Omega \backslash \omega^{+}\right)} \leq\left\|u^{\varepsilon}-u^{0, r e c}\right\|_{\mathrm{H}^{1}\left(\Omega \backslash \omega^{+}\right)}+\left\|u^{0, r e c}-u_{2}^{r e c}\left(\theta_{2}\right)\right\|_{\mathrm{H}^{1}\left(\Omega \backslash \omega^{+}\right)} .
$$

The first norm is bounded by $C \varepsilon^{1 / 2}$; this follows from [28]. The second norm can be bounded by

$$
\begin{aligned}
\left\|u^{0, r e c}-u_{2}^{r e c}\left(\theta_{2}\right)\right\|_{\mathrm{H}^{1}\left(\Omega \backslash \omega^{+}\right)} \leq & \left\|u^{0}-u_{2}^{0}\left(\theta_{2}\right)\right\|_{\mathrm{H}^{1}\left(\Omega \backslash \omega^{+}\right)} \\
& +\varepsilon\left\|\sum_{j=1}^{d} \chi^{j}\left(x, \frac{x}{\varepsilon}\right)\left(\frac{\partial u^{0}(x)}{\partial x_{j}}-\frac{\partial u_{2}^{0}(x)}{\partial x_{j}}\right)\right\|_{\mathrm{H}^{1}\left(\Omega \backslash \omega^{+}\right)} .
\end{aligned}
$$

Each of the terms can be bounded by $C \varepsilon$, using the Caccioppoli inequality on the difference $u^{0}-u_{2}^{0}\left(\theta_{2}\right)$ and Lemma 3.5.

4. Fully discrete optimization based coupling method. In this section, we derive a numerical method to solve the optimization based fine scale and coarse scale problems. To fully resolve the fine scales in $\omega_{1}$, we need a triangulation with mesh size that resolves the fine scale, whereas the triangulation of $\Omega \backslash \omega_{1}$ can be coarse and independent of the smallest scale, thanks to numerical homogenization techniques. In order to allow for flexible meshing, we do not impose continuity of the numerical homogenization method on $\Gamma_{1}$. Here we choose to use a discontinuous Galerkin method on $\omega_{2}$ and a continuous FEM on $\omega_{1}$.

In what follows, we restrict the family of problems (2.1) to homogeneous Dirichlet problems; i.e., we set $g_{D}=0$ and $\Gamma_{N}=\{\emptyset\}$. We denote by $H_{D}^{1}\left(\omega_{i}\right)$ the set of functions in $H^{1}\left(\omega_{i}\right)$ that vanish on $\partial \omega_{i} \cap \Gamma_{D}$ for $i=1,2$.

Further, we assume that the strong Cauchy-Schwarz lemma, Lemma A.3, and its discrete version, Lemma A.7, hold.

Copyright $@$ by SIAM. Unauthorized reproduction of this article is prohibited. 
Numerical method for the fine scale problem. Let $\left\{\mathcal{T}_{\tilde{h}}\right\}_{\tilde{h}}$ be a family of partitions over $\omega_{1}$ in simplicial or quadrilateral elements, with mesh size $\tilde{h} \ll \varepsilon$, where $\tilde{h}=$ $\max _{K \in \mathcal{T}_{\tilde{h}}} h_{K}$, and $h_{K}$ is the diameter of the element $K$. In addition, we suppose that the family of partitions $\left\{\mathcal{T}_{\tilde{h}}\right\}_{\tilde{h}}$ is admissible and shape regular [14]:

(T1) Admissible. $\bar{\omega}_{1}=\cup_{K \in \mathcal{T}_{h}} K$, and the intersection of two elements is empty, a vertex, or a common face.

(T2) Shape regular. There exists $\sigma>0$ such that $h_{K} / \rho_{K} \leq \sigma$ for all $K \in \mathcal{T}_{\tilde{h}}$ and for all $\mathcal{T}_{\tilde{h}} \in\left\{\mathcal{T}_{\tilde{h}}\right\}_{\tilde{h}}$, where $\rho_{K}$ is the diameter of the largest circle contained in the element $K$.

For simplicity, we consider, for each partition $\mathcal{T}_{\tilde{h}} \in\left\{\mathcal{T}_{\tilde{h}}\right\}_{\tilde{h}}$, a piecewise FE in $\omega_{1}$, given by

$$
V_{D}^{1}\left(\omega_{1}, \mathcal{T}_{\tilde{h}}\right)=\left\{w \in H_{D}^{1}\left(\omega_{1}\right) \mid w_{\left.\right|_{K}} \in \mathcal{R}^{1}(K) \forall K \in \mathcal{T}_{\tilde{h}}\right\},
$$

where $\mathcal{R}^{1}$ is the space of piecewise polynomials on $K$. Further, we denote by $V_{0}^{1}\left(\omega_{1}, \mathcal{T}_{\tilde{h}}\right)$ the functions in $V_{D}^{1}\left(\omega_{1}, \mathcal{T}_{\tilde{h}}\right)$ that vanish on $\partial \omega_{1}$.

Let $u_{1, \tilde{h}}$ be the numerical approximation of $u_{1}^{\varepsilon}$ satisfying (2.3) for $i=1$. We can decompose $u_{1, \tilde{h}}$ into $u_{1, \tilde{h}}=u_{1,0, \tilde{h}}+v_{1, \tilde{h}}$, where $v_{1, \tilde{h}} \in V_{D}^{1}\left(\omega_{1}, \mathcal{T}_{\tilde{h}}\right)$ is obtained by the optimization method and $u_{1,0, \tilde{h}} \in V_{0}^{1}\left(\omega_{1}, \mathcal{T}_{\tilde{h}}\right)$ is the solution of

$$
B_{1}\left(u_{1,0, \tilde{h}}, w_{1, \tilde{h}}\right)=F_{1}\left(w_{1, \tilde{h}}\right) \quad \forall w_{1, \tilde{h}} \in V_{0}^{1}\left(\omega_{1}, \mathcal{T}_{\tilde{h}}\right),
$$

where the right-hand side $F_{1}$ is given by

$$
F_{1}\left(w_{1, \tilde{h}}\right)=\int_{\omega_{1}} f w_{1, \tilde{h}} \mathrm{~d} x
$$

Thanks to the Poincaré inequality, the bilinear form $B_{1}$ is coercive and bounded over $V_{0}^{1}\left(\omega_{1}, \mathcal{T}_{\tilde{h}}\right)$; the existence and uniqueness of $u_{1,0, \tilde{h}}$ follows. We note that a quadrature formula should be considered for the bilinear form $B_{1}$ and for the right-hand side $F_{1}$.

Discontinuous Galerkin $(D G)$ method for the coarse scale problem. Let $\left\{\mathcal{T}_{H}\right\}_{H}$ be a family of partitions over $\omega_{2}$, with discontinuity in $\Gamma_{1}$ and mesh size $H=$ $\max _{K \in \mathcal{T}_{H}} h_{K}$; further, we assume that the family of partitions $\left\{\mathcal{T}_{H}\right\}_{H}$ is shape regular (T2). For each partition $\mathcal{T}_{H} \in\left\{\mathcal{T}_{H}\right\}_{H}$, we denote by $E$ the set of $(d-1)$-dimensional elements of $\mathcal{T}_{H}$ that form the boundary $\Gamma_{1}$-it will be edges (for $d=2$ ) or faces (for $d=3$ ). Further, assume that the set $E$ is composed of the smallest common interface between two elements $K_{+}$and $K_{-}$of $\mathcal{T}_{H}$, with intersection in $\Gamma_{1}$; that is, $e$ is in $E$ if $e=\min K_{+} \cap K_{-}$and $e \subset \Gamma_{1}$. As the solutions of problem (2.3) for $i=2$ are assumed to be continuous in $\omega_{2} \backslash \Gamma_{1}$, we construct a piecewise FE space as

$$
V_{D}^{1}\left(\omega_{2}, \mathcal{T}_{H}\right)=\left\{v \in H_{D}^{1}\left(\omega_{2} \backslash \Gamma_{1}\right) \cap L^{2}\left(\omega_{2}\right) \mid v_{\left.\right|_{K}} \in \mathcal{R}^{1}(K) \forall K \in \mathcal{T}_{H}\right\},
$$

and we denote by $V_{0}^{1}\left(\omega_{2}, \mathcal{T}_{H}\right)$ the set of functions of $V_{D}^{1}\left(\omega_{2}, \mathcal{T}_{H}\right)$ that vanish over $\partial \omega_{2}$. For $v \in V_{D}^{1}\left(\omega_{2}, \mathcal{T}_{H}\right)$, we consider its average $\{\cdot\}$ and its jump $\llbracket \cdot \rrbracket$ given by

$$
\{v\}=\frac{1}{2}\left(v_{+}+v_{-}\right) \quad \text { and } \quad \llbracket v \rrbracket=v_{+} n_{+}+v_{-} n_{-},
$$

where $v_{ \pm}:=\left.v\right|_{K_{ \pm}}$denotes the trace of $v$ from within $K_{ \pm}$and $n_{ \pm}$stands for the unit outward normal in $K_{ \pm}$.

Quadrature formula. For piecewise FE spaces, a quadrature formula is given by the pair $\left(x_{K},|K|\right)$, where $x_{K}$ is the barycenter of $K$. The sampling domain of size $\delta$ around each point $x_{K}$ is denoted by $K_{\delta}=x_{K}+\delta[-1 / 2,1 / 2]^{2}$. 
The numerically homogenized tensor $a_{2}^{0, h}\left(x_{K}\right)$ around the quadrature point $x_{K}$ is obtained using numerical solutions of microproblems defined in the sampling domains $K_{\delta}$; we note that a numerical approximation of $f^{0}$ can be obtained similarly. Let us consider a partition $\mathcal{T}_{h}$ of $K_{\delta}$ in simplicial or quadrilateral elements $K$ of diameter $h_{K}$; the mesh size is $h=\max _{K \in \mathcal{T}_{h}} h_{K}$, and as the fine scales should be resolved in $K_{\delta}$, we impose $h<\varepsilon$. The piecewise micro-FE space is given by

$$
S^{1}\left(K_{\delta}, \mathcal{T}_{h}\right)=\left\{v^{h} \in W\left(K_{\delta}\right) \mid v_{\left.\right|_{K}}^{h} \in \mathcal{R}^{1}(K) \forall K \in \mathcal{T}_{h}\right\},
$$

where $W\left(K_{\delta}\right)$ depends on the boundary conditions imposed on the microproblems; $W\left(K_{\delta}\right)=H_{0}^{1}\left(K_{\delta}\right)$ for Dirichlet coupling, or $W\left(K_{\delta}\right)=W_{\text {per }}^{1}\left(K_{\delta}\right)$ for periodic coupling. We introduce discrete microproblems: find $\psi_{K_{\delta}}^{i, h} \in S^{1}\left(K_{\delta}, \mathcal{T}_{h}\right), i=1, \ldots, d$, the solution of

$$
\int_{K_{\delta}} a_{2}^{\varepsilon}(x) \nabla \psi_{K_{\delta}}^{i, h} \cdot \nabla w_{j}^{h} \mathrm{~d} x=-\int_{K_{\delta}} a_{2}^{\varepsilon}(x) e_{i} \cdot \nabla w_{j}^{h} \mathrm{~d} x \quad \forall w_{j}^{h} \in S^{1}\left(K_{\delta}, \mathcal{T}_{h}\right) .
$$

The numerically homogenized tensor at a quadrature point $x_{K}$ in a macroelement $K$ is computed by

$$
a_{2}^{0, h}\left(x_{K}\right)=\frac{1}{\left|K_{\delta}\right|} \int_{K_{\delta}} a_{2}^{\varepsilon}(x)\left(I+\nabla \psi_{K_{\delta}}^{h}\right) \mathrm{d} x,
$$

where $\nabla \psi_{K_{\delta}}^{h}=\left(\nabla \psi_{K_{\delta}}^{1, h}, \ldots, \nabla \psi_{K_{\delta}}^{d, h}\right)$. Following [4], we define a DG macrobilinear form $B_{2, H}(\cdot, \cdot)$ over $V_{D}^{1}\left(\omega_{2}, \mathcal{T}_{H}\right) \times V_{D}^{1}\left(\omega_{2}, \mathcal{T}_{H}\right)$ by

$$
\begin{aligned}
& B_{2, H}\left(v_{2, H}, w_{2, H}\right)= \sum_{K \in \mathcal{T}_{H}}|K| a_{2}^{0, h}\left(x_{K}\right) \nabla v_{2, H}\left(x_{K}\right) \cdot \nabla w_{2, H}\left(x_{K}\right) \\
&+\sum_{e \in E} \int_{e} \mu_{e} \llbracket v_{2, H} \rrbracket \llbracket w_{2, H} \rrbracket \mathrm{d} s \\
&-\sum_{e \in E} \int_{e}\left(\left\{a_{2}^{0, h}\left(x_{K}\right) \nabla v_{2, H}\left(x_{K}\right)\right\} \llbracket w_{2, H} \rrbracket\right. \\
&\left.+\left\{a_{2}^{0, h}\left(x_{K}\right) \nabla w_{2, H}\left(x_{K}\right)\right\} \llbracket v_{2, H} \rrbracket\right) \mathrm{d} s,
\end{aligned}
$$

where the functions $\mu_{e}$ stand for weighting functions that penalize the jumps of $v_{2, H}$ and $w_{2, H}$ over the element $e$ in $E$. They are given by

$$
\mu_{e}=\kappa h_{e}^{-1},
$$

with $\kappa>0$, and $h_{e}$ is the size of the interface $e$.

The numerical homogenized solution $u_{2, H}$ is split into $u_{2, H}=u_{2,0, H}+v_{2, H}$, where $v_{2, H} \in V_{D}^{1}\left(\omega_{2}, \mathcal{T}_{H}\right)$ is given by the coupling and $u_{2,0, H} \in V_{0}^{1}\left(\omega_{2}, \mathcal{T}_{H}\right)$ by solving

$$
B_{2, H}\left(u_{2,0, H}, w_{2, H}\right)=F_{2}\left(w_{2, H}\right) \quad \forall w_{2, H} \in V_{0}^{1}\left(\omega_{2}, \mathcal{T}_{H}\right) .
$$

The right-hand side $F_{2}$ is given by

$$
F_{2}\left(w_{2, H}\right)=\sum_{K \in \mathcal{T}_{H}}|K| f\left(x_{K}\right) w_{2, H}\left(x_{K}\right) .
$$

Copyright (c) by SIAM. Unauthorized reproduction of this article is prohibited. 
Remark 4.1. Considering the nonhomogeneous Dirichlet boundary condition $g_{D} \neq$ 0 on $\Gamma_{D}$ and the Neumann condition on $\Gamma_{N} \neq\{0\}$ leads to some additional terms in the right-hand sides $F_{1}$ and $F_{2}$ of problems (4.1) and (4.6), respectively. In particular, one should construct a lifting of the Dirichlet data as explained in section 2.

Remark 4.2. Higher order FE spaces can be considered, and we note that the macro-FEM over $\omega_{1}$ and the micro-FEM over the sampling domains can be easily generalized to higher order FEMs. For the DG-FE-HMM some work needs to be done on the average of the fluxes, and we refer the reader to [4].

4.1. Numerical algorithm. In this section, we state the discrete coupling, give the algorithm, and present the main convergence results. The well-posedness and the proofs of the error estimates are given in the next sections.

The solution $\left(u_{1, \tilde{h}}, u_{2, H}\right) \in V_{D}^{1}\left(\omega_{1}, \mathcal{T}_{\tilde{h}}\right) \times V_{D}^{1}\left(\omega_{2}, \mathcal{T}_{H}\right)$ satisfies

$\min _{\mu_{1, \tilde{h}}, \mu_{2, H}} \frac{1}{2}\left\|u_{1, \tilde{h}}\left(\mu_{1, \tilde{h}}\right)-u_{2, H}\left(\mu_{2, H}\right)\right\|_{\mathrm{L}^{2}\left(\omega_{0}\right)}^{2}$ subject to $\left\{\begin{array}{l}B_{1}\left(u_{1, \tilde{h}}, w_{1, \tilde{h}}\right)=F_{1}\left(w_{1, \tilde{h}}\right), \\ B_{2, H}\left(u_{2, H}, w_{2, H}\right)=F_{2}\left(w_{2, H}\right)\end{array}\right.$

for all $w_{1, \tilde{h}} \in V_{0}^{1}\left(\omega_{1}, \mathcal{T}_{\tilde{h}}\right)$ and $w_{2, H} \in V_{0}^{1}\left(\omega_{2}, \mathcal{T}_{H}\right)$. Introducing discrete Lagrange multipliers $\lambda_{1, \tilde{h}} \in V_{0}^{1}\left(\omega_{1}, \mathcal{T}_{\tilde{h}}\right)$ and $\lambda_{2, H} \in V_{0}^{1}\left(\omega_{2}, \mathcal{T}_{H}\right)$ for each of the constraints leads to a discrete optimality system: find $\left(v_{1, \tilde{h}}, \lambda_{1, \tilde{h}}, v_{2, H}, \lambda_{2, H}\right) \in V_{D}^{1}\left(\omega_{1}, \mathcal{T}_{\tilde{h}}\right) \times V_{0}^{1}\left(\omega_{1}, \mathcal{T}_{\tilde{h}}\right) \times$ $V_{D}^{1}\left(\omega_{2}, \mathcal{T}_{H}\right) \times V_{0}^{1}\left(\omega_{2}, \mathcal{T}_{H}\right)$ satisfying

$$
\begin{aligned}
\int_{\omega_{0}}\left(v_{1, \tilde{h}}-v_{2, H}\right) w_{1, \tilde{h}} \mathrm{~d} x-B_{1}\left(w_{1, \tilde{h}}, \lambda_{1, \tilde{h}}\right) & =-\int_{\omega_{0}}\left(u_{1,0, \tilde{h}}-u_{2,0, H}\right) w_{1, \tilde{h}} \mathrm{~d} x \\
B_{1}\left(v_{1, \tilde{h}}, \xi_{1, \tilde{h}}\right) & =F_{1}\left(\xi_{1, \tilde{h}}\right)-B_{1}\left(u_{1,0, \tilde{h}}, \xi_{1, \tilde{h}}\right) \\
\int_{\omega_{0}}\left(v_{2, H}-v_{1, \tilde{h}}\right) w_{2, H} \mathrm{~d} x-B_{2, H}\left(w_{2, H}, \lambda_{2, H}\right) & =\int_{\omega_{0}}\left(u_{1,0, \tilde{h}}-u_{2,0, H}\right) w_{2, H} \mathrm{~d} x \\
B_{2, H}\left(v_{2, H}, \xi_{2, H}\right) & =F_{2}\left(\xi_{2, H}\right)-B_{2, H}\left(u_{2,0, H}, \xi_{2, H}\right)
\end{aligned}
$$

for all $w_{1, \tilde{h}} \in V_{D}^{1}\left(\omega_{1}, \mathcal{T}_{\tilde{h}}\right), \xi_{1, \tilde{h}} \in V_{0}^{1}\left(\omega_{1}, \mathcal{T}_{\tilde{h}}\right), w_{2, H} \in V_{D}^{1}\left(\omega_{2}, \mathcal{T}_{H}\right), \xi_{2, H} \in V_{0}^{1}\left(\omega_{2}, \mathcal{T}_{H}\right)$

The optimality system (4.7)-(4.10) can be written in matrix form, for the unknown vector $U=\left(v_{1, \tilde{h}}, v_{2, H}, \lambda_{1, \tilde{h}}, \lambda_{2, H}\right)^{\top}$, as

$$
\left(\begin{array}{cc}
M & -B^{\top} \\
B & 0
\end{array}\right) U=G
$$

The algorithm for the numerical coupling method is the following:

1. Find $u_{1,0, \tilde{h}} \in V_{0}^{1}\left(\omega_{1}, \mathcal{T}_{\tilde{h}}\right)$ satisfying

$$
B_{1}\left(u_{1,0, \tilde{h}}, w_{1, \tilde{h}}\right)=F_{1}\left(w_{1, \tilde{h}}\right) \quad \forall w_{1, \tilde{h}} \in V_{0}^{1}\left(\omega_{1}, \mathcal{T}_{\tilde{h}}\right) .
$$

2. Find $u_{2,0, H} \in V_{0}^{1}\left(\omega_{2}, \mathcal{T}_{H}\right)$ satisfying

$$
B_{2, H}\left(u_{2,0, H}, w_{2, H}\right)=F_{2}\left(w_{2, H}\right) \quad \forall w_{2, H} \in V_{0}^{1}\left(\omega_{2}, \mathcal{T}_{H}\right) .
$$

3. Find $v_{1, \tilde{h}} \in V_{D}^{1}\left(\omega_{1}, \mathcal{T}_{\tilde{h}}\right)$ and $v_{2, H} \in V_{D}^{1}\left(\omega_{2}, \mathcal{T}_{H}\right)$ by solving the saddle point problem (4.11). 
We state the two main convergence results for the fully discrete coupling. The optimization based method relies on the DG-FE-HMM; thus one should expect to find the DG-FE-HMM error in the a priori estimates. The DG-FE-HMM is split into a macro-, a micro-, and a modeling error:

$$
e_{H M M} \leq e_{M A C}+e_{M I C}+e_{M O D}
$$

The macro- and microerrors correspond to FE errors due to the choice of macroand micro-FEM, respectively. The modeling error is due to the upscaling procedure and will be influenced by the choice of boundary conditions for (4.2), the size of the sampling domain $\delta$, and whether we consider collocation in the macro- and microbilinear forms of the slow variable $x$ to the quadrature points in the tensor $a_{2}^{\varepsilon}$. Details about the DG-FE-HMM error are given in section 5 . Let $\left(\theta_{1, \tilde{h}}, \theta_{2, H}\right)$ be the discrete couple of boundary conditions given by the minimization problem (2.7). We recall the notation

$$
\begin{gathered}
u_{1, \tilde{h}}\left(\theta_{1, \tilde{h}}\right) \text { denotes the fine scale numerical solution in } \omega_{1}, \\
u_{2, H}\left(\theta_{2, H}\right) \text { denotes the coarse scale numerical solution in } \omega_{2} \text {. }
\end{gathered}
$$

The coupling solution, denoted by $\bar{u}_{\tilde{h} H}$, is defined as

$$
\bar{u}_{\tilde{h} H}= \begin{cases}u_{1, \tilde{h}}\left(\theta_{1, \tilde{h}}\right) & \text { in } \omega^{+}, \\ u_{2, H}^{r e c}\left(\theta_{2, H}\right) & \text { in } \Omega \backslash \omega^{+},\end{cases}
$$

where $u_{2, H}^{r e c}\left(\theta_{2, H}\right)$ corresponds to the reconstructed coarse scale solution $u_{2, H}\left(\theta_{2, H}\right)$ and is defined by

$$
u_{2, H}^{r e c}(x)=u_{2, H}(x)+\sum_{j=1}^{d} \psi_{K_{\varepsilon}}^{j, h}(x) \frac{\partial u_{2, H}}{\partial x_{j}}(x), \quad x \in K,
$$

where $\psi_{K_{\varepsilon}}^{j, h}$ are the micro solutions of (4.2). As the reconstructed numerical solution might be discontinuous across elements in $\omega_{2}$, we consider a broken $H^{1}$ seminorm,

$$
\|v\|_{\mathrm{H}^{1}(\Omega)}^{2}:=\sum_{K \in \mathcal{T}_{h}\left(\omega^{+}\right)}\|\nabla v\|_{\mathrm{L}^{2}(K)}^{2}+\sum_{K \in \mathcal{T}_{H}\left(\Omega \backslash \omega^{+}\right)}\|\nabla v\|_{\mathrm{L}^{2}(K)}^{2} .
$$

We next state our main convergence result for the optimization based numerical solution. We first have an error estimate in the fine scale region.

Theorem 4.3 (a priori error analysis in $\omega^{+}$). Let $\varepsilon_{0}$ be given by the strong CauchySchwarz lemma, Lemma A.3, and consider $\varepsilon \leq \varepsilon_{0}$. Let $u^{\varepsilon}$ and $u^{0}$ be the exact solutions of problems (2.1) and (3.3), respectively, and let $\bar{u}_{\tilde{h} H}$ be the numerical solution of the coupling (4.14). Assume $u^{\varepsilon} \in H^{s+1}(\Omega)$, with $s \leq 1, u^{0} \in H^{2}\left(\omega_{2}\right)$, and assume that (3.4) holds; then

$$
\left\|u^{\varepsilon}-u_{1, \tilde{h}}\left(\theta_{1, \tilde{h}}\right)\right\|_{\overline{\mathrm{H}}^{1}\left(\omega^{+}\right)} \leq C_{1} \tilde{h}^{s}\left|u^{\varepsilon}\right|_{H^{s+1}\left(\omega_{1}\right)}+\frac{C_{2}}{\tau-\tau^{+}}\left(\tilde{h}^{s+1}\left|u^{\varepsilon}\right|_{H^{s+1}\left(\omega_{1}\right)}+\varepsilon+e_{H M M, L^{2}}\right),
$$

where the constants are independent of $\varepsilon, H, \tilde{h}$, and $h$. The DG-FE-HMM error is given in Lemmas 5.2, 5.3, and 5.5.

Next, we state error estimates in the coarse scale region for the optimization based numerical solution with correctors. 
Theorem 4.4 (error estimates in $\Omega \backslash \omega^{+}$). Let $u^{\varepsilon}$ be the exact solution of problem (2.1) and $\bar{u}_{\tilde{h} H}$ be the numerical solution of the coupling (4.14). Let $a_{2}^{\varepsilon}(x)=$ $a_{2}(x, x / \varepsilon)$, where $a_{2}(x, y)$ is $Y$-periodic in $y$ and satisfies $a_{2}(x, y) \in \mathcal{C}\left(\bar{\omega}_{2} ; L_{p e r}^{\infty}(Y)\right)$. Let $\psi_{K_{\varepsilon}}^{j}(x) \in W_{p e r}^{1}\left(K_{\varepsilon}\right), j=1, \ldots, d$. If, in addition, $u^{\varepsilon} \in H^{2}(\Omega), u_{2}^{0}\left(\theta_{2}\right) \in H^{2}\left(\omega_{2}\right)$, $u_{1}^{\varepsilon} \in H^{s+1}\left(\omega_{1}\right)$, with $s \leq 1$, and $\psi_{K_{\varepsilon}}^{j}(x) \in W^{1, \infty}\left(K_{\varepsilon}\right), j=1, \ldots, d$. It holds that

$$
\begin{aligned}
\left\|u_{2}^{r e c}\left(\theta_{2}\right)-u_{2, H}^{r e c}\left(\theta_{2, H}\right)\right\|_{\overline{\mathrm{H}}^{1}\left(\Omega \backslash \omega^{+}\right)} \leq & C_{1} \varepsilon^{1 / 2}+C_{2}\left(\frac{h}{\varepsilon}\right)+C_{3} H\left|u_{2}^{0}\right|_{H^{2}\left(\omega_{2}\right)} \\
& +\frac{C_{4}}{\tau^{+}}\left(\tilde{h}^{s+1}\left|u_{1}^{\varepsilon}\right|_{H^{s+1}\left(\omega_{1}\right)}+\varepsilon+H^{2}\left|u_{2}^{0}\right|_{H^{2}\left(\omega_{2}\right)}\right),
\end{aligned}
$$

where the constants are independent of $H, \tilde{h}, h$, and $\varepsilon$.

Both theorems will be proved in section 5 . We first discuss the well-posedness of the numerical method.

4.2. Well-posedness. In this subsection, we prove the well-posedness of the discrete coupling problem. The well-posedness of the DG optimization based coupling method can be established using Brezzi's theory [12] and the well-posedness of problems (4.12) and (4.13). The Lax-Milgram lemma implies the existence and uniqueness of $u_{1,0, \tilde{h}} \in V_{0}^{1}\left(\omega_{1}, \mathcal{T}_{\tilde{h}}\right)$.

Due to the discontinuity in $\mathcal{T}_{H}$, the space $V_{0}^{1}\left(\omega_{2}, \mathcal{T}_{H}\right)$ is not a subspace of $H_{0}^{1}\left(\omega_{2}\right)$; however, it will lie in the piecewise Sobolev space

$$
H^{2}\left(\mathcal{T}_{H}\right):=\prod_{K \in \mathcal{T}_{H}} H^{2}(K)=\left\{v \in L^{1}\left(\omega_{2}\right) \mid v_{\mid K} \in H^{2}(K) \forall K \in \mathcal{T}_{H}\right\}
$$

Suppose that the exact solution $u_{2,0}$ of problem (2.5) is in the space $H_{0}^{1}\left(\omega_{2}\right) \cap H^{2}\left(\omega_{2}\right)$. We define the proper space for the analysis as $V\left(\omega_{2}\right):=V_{0}^{1}\left(\omega_{2}, \mathcal{T}_{H}\right)+H_{0}^{1}\left(\omega_{2}\right) \cap$ $H^{2}\left(\omega_{2}\right) \subset H^{2}\left(\mathcal{T}_{H}\right)$; see discussions in $[8,18]$. The space $V\left(\omega_{2}\right)$ is equipped with the norm

$$
\|v\|_{\omega_{2}}:=\left(\|\nabla v\|_{\mathrm{L}^{2}\left(\omega_{2}\right)}^{2}+\sum_{K \in \mathcal{T}_{H}} h_{K}^{2}|v|_{2, K}^{2}+|v|_{*}^{2}\right)^{1 / 2}
$$

where

$$
\|\nabla v\|_{\mathrm{L}^{2}\left(\omega_{2}\right)}^{2}=\sum_{K \in \mathcal{T}_{H}}|v|_{1, K}^{2}, \quad|v|_{2, K}^{2}=\sum_{|r|=2}\left\|\partial^{r} v\right\|_{\mathrm{L}^{2}(K)}^{2}, \quad \text { and }|v|_{*}^{2}=\sum_{e \in E}\left\|\mu_{e}^{1 / 2} \llbracket v \rrbracket\right\|_{\mathrm{L}^{2}(e)}^{2} .
$$

One can prove that (4.15) is a norm over $V\left(\omega_{2}\right)$, using the discrete Poincaré-Friedrichs inequality [12],

$$
\|v\|_{\mathrm{L}^{2}\left(\omega_{2}\right)}^{2} \leq C\left(\|\nabla v\|_{\mathrm{L}^{2}\left(\omega_{2}\right)}^{2}+|v|_{*}^{2}\right) .
$$

Thanks to local inverse inequalities [14], restricting $V\left(\omega_{2}\right)$ to $V_{0}^{1}\left(\omega_{2}, \mathcal{T}_{H}\right)$ reduces the norm (4.15) to

$$
\|v\|_{\omega_{2}}=\left(\|\nabla v\|_{\mathrm{L}^{2}\left(\omega_{2}\right)}^{2}+|v|_{*}^{2}\right)^{1 / 2}
$$

Copyright (c) by SIAM. Unauthorized reproduction of this article is prohibited. 
Proposition 4.5. There exists a value $\kappa_{0}$ that depends only on (2.2), the shape regularity of $\mathcal{T}_{H}$, and the dimension $d$ such that for all $\kappa \geq \kappa_{0}, \kappa$ being defined in (4.5), the bilinear form $B_{2, H}(4.4)$ is stable in $V_{0}^{1}\left(\omega_{2}, \mathcal{T}_{H}\right)$; i.e.,

$$
B_{2, H}\left(v_{H}, v_{H}\right) \geq C_{1}\left\|v_{H}\right\|^{2} \quad \forall v_{H} \in V_{0}^{1}\left(\omega_{2}, \mathcal{T}_{H}\right) .
$$

Furthermore, the bilinear form is bounded; i.e.,

$$
B_{2, H}\left(v_{H}, w_{H}\right) \leq C_{2}\left\|v_{H}\right\|\|\| w_{H}\|\| \quad \forall v_{H}, w_{H} \in V_{0}^{1}\left(\omega_{2}, \mathcal{T}_{H}\right) .
$$

The constants $C_{1}$ and $C_{2}$ are independent of $H, \tilde{h}, h$, and $\varepsilon$.

Proof. For the proof, see [4, Lemmas 4.3, 4.4, and 5.18].

TheOREM 4.6. Let assumption (2.2) hold. Then there exists a unique solution $u_{1,0, \tilde{h}} \in V_{0}^{1}\left(\omega_{1}, \mathcal{T}_{\tilde{h}}\right)$ of problem (4.12) which satisfies

$$
\left\|u_{1,0, \tilde{h}}\right\|_{\mathrm{H}^{1}\left(\omega_{1}\right)} \leq C_{1}\left\|F_{1}\right\|_{\mathrm{H}^{-1}\left(\omega_{1}\right)},
$$

with a constant $C_{1}$ independent of $H, \tilde{h}$, and $\varepsilon$.

Moreover, let $\kappa_{0}$ be given by Proposition 4.5. Then, the problem (4.13) admits a unique solution $u_{2,0, H} \in V_{0}^{1}\left(\omega_{2}, \mathcal{T}_{H}\right)$, and it holds that

$$
\left\|u_{2,0, H}\right\| \leq C_{2}\left\|F_{2}\right\|_{\mathrm{H}^{-1}\left(\omega_{2}\right)}
$$

where the constant $C_{2}$ is independent of $H, h, \tilde{h}$, and $\varepsilon$.

Proof. The existence and uniqueness of $u_{1,0, \tilde{h}}$ and $u_{2,0, H}$ follows from the LaxMilgram lemma and Proposition 4.5.

We introduce $V^{1}\left(\Gamma_{i}\right)$ as the set of functions $\mu_{i} \in \mathcal{U}^{i}$ that are piecewise polynomials on the elements over $\Gamma_{i}, i=1,2$. Let us write system (4.7)-(4.10) in terms of the discrete virtual controls $\theta_{1, \tilde{h}}$ and $\theta_{2, H}$ : find $\left(\theta_{1, \tilde{h}}, \lambda_{1, \tilde{h}}, \theta_{2, H}, \lambda_{2, H}\right) \in$ $V^{1}\left(\Gamma_{1}\right) \times V_{0}^{1}\left(\omega_{1}, \mathcal{T}_{\tilde{h}}\right) \times V^{1}\left(\Gamma_{2}\right) \times V_{0}^{1}\left(\omega_{2}, \mathcal{T}_{H}\right)$ satisfying

$$
\begin{aligned}
\pi\left(\left(\theta_{1, \tilde{h}}, \theta_{2, H}\right),\left(\mu_{1, \tilde{h}}, \mu_{2, H}\right)\right)-B\left(\left(\mu_{1, \tilde{h}}, \mu_{2, H}\right),\left(\lambda_{1, \tilde{h}}, \lambda_{2, H}\right)\right) & =G\left(\mu_{1, \tilde{h}}, \mu_{2, H}\right), \\
B\left(\left(\theta_{1, \tilde{h}}, \theta_{2, H}\right),\left(\xi_{1, \tilde{h}}, \xi_{2, H}\right)\right) & =0
\end{aligned}
$$

for all $\left(\mu_{1, \tilde{h}}, \mu_{2, H}\right) \in V^{1}\left(\Gamma_{1}\right) \times V^{1}\left(\Gamma_{2}\right)$ and $\left(\xi_{1, \tilde{h}}, \xi_{2, H}\right) \in V_{0}^{1}\left(\omega_{1}, \mathcal{T}_{\tilde{h}}\right) \times V_{0}^{1}\left(\omega_{2}, \mathcal{T}_{H}\right)$. The forms $\pi, B$, and $G$ are defined by

$$
\begin{aligned}
\pi\left(\left(\theta_{1, \tilde{h}}, \theta_{2, H}\right),\left(\mu_{1, \tilde{h}}, \mu_{2, H}\right)\right) & =\int_{\omega_{0}}\left(v_{1, \tilde{h}}\left(\theta_{1, \tilde{h}}\right)-v_{2, H}\left(\theta_{2, H}\right)\right)\left(v_{1, \tilde{h}}\left(\mu_{1, \tilde{h}}\right)-v_{2, H}\left(\mu_{2, H}\right)\right) \mathrm{d} x, \\
B\left(\left(\theta_{1, \tilde{h}}, \theta_{2, H}\right),\left(\xi_{1, \tilde{h}}, \xi_{2, H}\right)\right) & =B_{1}\left(\theta_{1, \tilde{h}}, \xi_{1, \tilde{h}}\right)+B_{2, H}\left(\theta_{2, H}, \xi_{2, H}\right), \\
G\left(\theta_{1, \tilde{h}}, \theta_{2, H}\right) & =-\int_{\omega_{0}}\left(u_{1,0, \tilde{h}}-u_{2,0, H}\right)\left(v_{1, \tilde{h}}\left(\theta_{1, \tilde{h}}\right)-v_{2, H}\left(\theta_{2, H}\right)\right) \mathrm{d} x .
\end{aligned}
$$

(Note that in order to avoid overloading of notation, we reuse the notation $\pi$ in the discrete context, which should not be confused with (2.8).)

To prove the well-posedness of system (4.17)-(4.18), we need to show that the following hold:

- The form $\pi$ is continuous and coercive on $V^{1}\left(\Gamma_{1}\right) \times V^{1}\left(\Gamma_{2}\right)$ equipped with the inner product $\pi$.

Copyright (c) by SIAM. Unauthorized reproduction of this article is prohibited. 
- The form $B$ is continuous and satisfies an inf-sup condition.

The continuity of $\pi$ can be easily obtained with the Cauchy-Schwarz inequality and the discrete Poincaré inequality (4.16).

The coercivity of $\pi$ can be proved similarly to the continuum case (cf. Lemma 2.2), as is done in the next lemma.

Lemma 4.7. Let $\varepsilon_{0}$ be given by the strong Cauchy-Schwarz lemma, Lemma A.3, and assume that $\varepsilon \leq \varepsilon_{0}$. Then, the form $\pi$ defines an inner product on $V^{1}\left(\Gamma_{1}\right) \times$ $V^{1}\left(\Gamma_{2}\right)$.

Proof. We will use the discrete Cauchy-Schwarz lemma, Lemma A.7, with the same $\varepsilon_{0}$ and $C_{s}$, to prove that $\pi$ is definite. Indeed, arguing as in Lemma 2.2, we assume that $\left(\mu_{1, \tilde{h}}, \mu_{2, H}\right)$ is such that

$$
\begin{aligned}
0 & =\pi\left(\left(\mu_{1, \tilde{h}}, \mu_{2, H}\right),\left(\mu_{1, \tilde{h}}, \mu_{2, H}\right)\right)=\left\|v_{1, \tilde{h}}\left(\mu_{1, \tilde{h}}\right)-v_{2, H}\left(\mu_{2, H}\right)\right\|_{\mathrm{L}^{2}\left(\omega_{0}\right)}^{2} \\
& \geq\left(1-C_{s}\right)\left(\left\|v_{1, \tilde{h}}\left(\mu_{1, \tilde{h}}\right)\right\|_{\mathrm{L}^{2}\left(\omega_{0}\right)}^{2}+\left\|v_{2, H}\left(\mu_{2, H}\right)\right\|_{\mathrm{L}^{2}\left(\omega_{0}\right)}^{2}\right) .
\end{aligned}
$$

As $C_{s}<1$, it holds that $\left\|v_{1, \tilde{h}}\left(\mu_{1, \tilde{h}}\right)\right\|_{\mathrm{L}^{2}\left(\omega_{0}\right)}=\left\|v_{2, H}\left(\mu_{2, H}\right)\right\|_{\mathrm{L}^{2}\left(\omega_{0}\right)}=0$, which implies that $v_{1, \tilde{h}}\left(\mu_{1, \tilde{h}}\right)=v_{2, H}\left(\mu_{2, H}\right)=0$ in $\omega_{0}$ and, in particular, $\mu_{1, \tilde{h}}=0$ and $\mu_{2, H}=0$.

Next, we prove the inf-sup condition for the bilinear form $B$.

Lemma 4.8. The form $B$ satisfies

$$
\sup _{\left(\mu_{1, \tilde{h}}, \mu_{2, H}\right)} \frac{B\left(\left(\mu_{1, \tilde{h}}, \mu_{2, H}\right),\left(\xi_{1, \tilde{h}}, \xi_{2, H}\right)\right)}{\left\|\left(\mu_{1, \tilde{h}}, \mu_{2, H}\right)\right\|_{L^{*}(\hat{U})}} \geq C\left(\left\|\xi_{1, \tilde{h}}\right\|_{\mathrm{H}^{1}\left(\omega_{1}\right)}+\left\|\xi_{2, H}\right\|_{\omega_{2}}\right)
$$

for all $\left(\xi_{1, \tilde{h}}, \xi_{2, H}\right) \in V_{0}^{1}\left(\omega_{1}, \mathcal{T}_{\tilde{h}}\right) \times V_{0}^{1}\left(\omega_{2}, \mathcal{T}_{H}\right)$. The constant $C$ is independent of $\varepsilon$.

Proof. Let $\left(\xi_{1, \tilde{h}}, \xi_{2, H}\right) \in V_{0}^{1}\left(\omega_{1}, \mathcal{T}_{\tilde{h}}\right) \times V_{0}^{1}\left(\omega_{2}, \mathcal{T}_{H}\right)$. By the definition of $B$, we have

$$
B\left(\left(\mu_{1, \tilde{h}}, \mu_{2, H}\right),\left(\xi_{1, \tilde{h}}, \xi_{2, H}\right)\right)=B_{1}\left(\mu_{1, \tilde{h}}, \xi_{1, \tilde{h}}\right)+B_{2, H}\left(\mu_{2, H}, \xi_{2, H}\right) .
$$

Take $\left(\mu_{1, \tilde{h}}, \mu_{2, H}\right)$ such that $v_{1, \tilde{h}}\left(\mu_{1, \tilde{h}}\right)=\xi_{1, \tilde{h}} \in V_{0}^{1}\left(\omega_{1}, \mathcal{T}_{h}\right)$ and $v_{2, H}\left(\mu_{2, H}\right)=\xi_{2, H} \in$ $V_{0}^{1}\left(\omega_{2}, \mathcal{T}_{H}\right)$. Then,

$B_{1}\left(\mu_{1, \tilde{h}}, \xi_{1, \tilde{h}}\right)=\int_{\omega_{1}} a_{1}^{\varepsilon} \nabla v_{1, \tilde{h}}\left(\mu_{1, \tilde{h}}\right) \cdot \nabla \xi_{1, \tilde{h}} \mathrm{~d} x=\int_{\omega_{1}} a_{1}^{\varepsilon} \nabla \xi_{1, \tilde{h}} \cdot \nabla \xi_{1, \tilde{h}} \mathrm{~d} x \geq C\left\|\xi_{1, \tilde{h}}\right\|_{\mathrm{H}^{1}\left(\omega_{1}\right)}^{2}$.

Similarly, by the coercivity of $B_{2, H}$, it holds that

$$
B_{2, H}\left(\mu_{2, H}, \xi_{2, H}\right) \geq C\left\|\xi_{2, H}\right\|_{\omega_{2}}^{2} .
$$

Thus,

$$
B\left(\left(\mu_{1, \tilde{h}}, \mu_{2, H}\right),\left(\xi_{1, \tilde{h}}, \xi_{2, H}\right)\right) \geq C\left(\left\|\xi_{1, \tilde{h}}\right\|_{\mathrm{H}^{1}\left(\omega_{1}\right)}+\left\|\xi_{2, H}\right\|_{\omega_{2}}\right)^{2},
$$

where the constant is independent of $H, h, \tilde{h}$, and $\varepsilon$. We can conclude since

$$
\begin{aligned}
\left\|\left(\mu_{1, \tilde{h}}, \mu_{2, H}\right)\right\|_{\mathrm{L}^{*}(\hat{U})} & \leq\left\|v_{1, \tilde{h}}\left(\mu_{1, \tilde{h}}\right)\right\|_{\mathrm{L}^{2}\left(\omega_{1}\right)}+\left\|v_{2, H}\left(\mu_{2, H}\right)\right\|_{\mathrm{L}^{2}\left(\omega_{2}\right)} \\
& \leq C\left(\left\|v_{1, \tilde{h}}\left(\mu_{1, \tilde{h}}\right)\right\|_{\mathrm{H}^{1}\left(\omega_{1}\right)}+\left\|v_{2, H}\left(\mu_{2, H}\right)\right\|_{\omega_{2}}\right) \\
& =C\left(\left\|\xi_{1, \tilde{h}}\right\|_{\mathrm{H}^{1}\left(\omega_{1}\right)}+\left\|\xi_{2, H}\right\|_{\omega_{2}}\right) .
\end{aligned}
$$

Copyright $@$ by SIAM. Unauthorized reproduction of this article is prohibited. 
5. Fully discrete error estimates. In this section, we derive error estimates for the fully discrete optimization based method. A postprocessing procedure is used on the coarse solution $u_{2, H}\left(\theta_{2, H}\right)$ to reach convergence to the exact solution $u^{\varepsilon}$. The norm considered is a broken $H^{1}$ seminorm, as we allow the corrected solution to be discontinuous across elements of $\Omega \backslash \omega$. The fully discrete analysis is then conducted for the error

$$
\left\|u^{\varepsilon}-\bar{u}_{\tilde{h} H}\right\|_{\overline{\mathrm{H}}^{1}(\Omega)}=\sum_{K \in \mathcal{T}_{h}\left(\omega^{+}\right)}\left\|\nabla\left(u^{\varepsilon}-\bar{u}_{\tilde{h}_{H}}\right)\right\|_{\mathrm{L}^{2}(K)}^{2}+\sum_{K \in \mathcal{T}_{H}\left(\Omega \backslash \omega^{+}\right)}\left\|\nabla\left(u^{\varepsilon}-\bar{u}_{\tilde{h}_{H}}\right)\right\|_{\mathrm{L}^{2}(K)}^{2},
$$

where the numerical solution of the coupling $\bar{u}_{\tilde{h} H}$ is given by (4.14). In the fully discrete analysis of the DG-FE-HMM method, the error between the homogenized solution and its approximation is decomposed into a macro-, a micro-, and a modeling error [2]. These errors will contribute to the a priori estimates of our method.

Remark 5.1. In section 3, the error estimates depend on the bound of the operator $Q$ (3.6). This bound was obtained in Lemma 3.4 using Caccioppoli inequalities. In the fully discrete case, we introduce a discrete operator $Q^{\tilde{h}, H}$, which is a discrete version of the operator $Q$, and the estimates will depend on $\left\|Q^{\tilde{h}, H}\right\|$. For conforming FE spaces, the norm of $Q^{\tilde{h}, H}$ is bounded independently of the mesh sizes $\tilde{h}, h$, and $H$; this can be seen by following along the lines of Lemma 3.4. For nonconforming meshes, we will assume that $\left\|Q^{\tilde{h}, H}\right\|$ is bounded independently of $\tilde{h}, h$, and $H$. In what follows, we will use the notation $P, U_{0}$, and $Q$, previously used in the continuous analysis, to denote the operators in the discrete analysis.

Preliminaries. We recall that $u^{0}$, the solution of (3.3), denotes a homogenized solution over $\omega_{2}$ with boundary condition on $\Gamma_{2}$ given by the trace of the physical solution $u^{\varepsilon}$ for a fixed $\varepsilon$. The DG-FE-HMM gives us an approximation $u^{H} \in V_{0}^{1}\left(\omega_{2}, \mathcal{T}_{H}\right)$ of the homogenized solution $u^{0}$. We state here the main results needed to bound $\left\|u^{0}-u^{H}\right\|_{\mathrm{L}^{2}\left(\omega_{2}\right)}$; for further details we refer the reader to $[1,2,3]$ and the references therein. We decompose the DG-FE-HMM error into the macro-, micro-, and modeling errors

$$
\left\|u^{0}-u^{H}\right\|_{L^{2}\left(\omega_{2}\right)} \leq e_{M A C}+e_{M I C}+e_{M O D} .
$$

Macroerror. We define $u_{H}^{0} \in V_{0}^{1}\left(\omega_{2}, \mathcal{T}_{H}\right)$ as the FEM approximation of the homogenized problem (3.3), i.e.,

$$
B_{2, H}^{0}\left(u_{H}^{0}, v_{H}\right)=F_{2}\left(v_{H}\right) \quad \forall v_{H} \in V_{0}^{1}\left(\omega_{2}, \mathcal{T}_{H}\right),
$$

where the bilinear form is given by

$$
\begin{aligned}
B_{2, H}^{0}\left(u_{H}, v_{H}\right)= & \sum_{K \in \mathcal{T}_{H}}|K| a_{2}^{0}\left(x_{K}\right) \nabla u_{H} \nabla v_{H}+\sum_{e \in E} \int_{e} \mu_{e} \llbracket u_{H} \rrbracket \llbracket v_{H} \rrbracket \mathrm{d} s \\
& -\sum_{e \in E} \int_{e}\left(\left\{a_{2}^{0} \nabla u_{H}\right\} \llbracket v_{H} \rrbracket+\left\{a_{2}^{0} \nabla v_{H}\right\} \llbracket u_{H} \rrbracket\right) \mathrm{d} s \quad \forall u_{H}, v_{H} \in V_{0}^{1}\left(\omega_{2}, \mathcal{T}_{H}\right) .
\end{aligned}
$$

The error can be formulated as

$$
\begin{aligned}
\left\|u^{0}-u^{H}\right\|_{\mathrm{L}^{2}\left(\omega_{2}\right)} & \leq\left\|u^{0}-u_{H}^{0}\right\|_{\mathrm{L}^{2}\left(\omega_{2}\right)}+\left\|u_{H}^{0}-u^{H}\right\|_{\mathrm{L}^{2}\left(\omega_{2}\right)} \\
& \leq\left\|u^{0}-u_{H}^{0}\right\|_{\mathrm{L}^{2}\left(\omega_{2}\right)}+\left\|u_{H}^{0}-u^{H}\right\|_{\omega_{2}},
\end{aligned}
$$

where the first norm is the macroerror and the second norm stands for the modeling and microerrors. 
To simplify the analysis, we make the following assumptions on the structure of the tensor $a_{2}^{\varepsilon}$ :

(H1) $a_{2}^{\varepsilon}(x)=a_{2}(x, x / \varepsilon)=a_{2}(x, y)$ is $Y$-periodic in $y$, and $\left.a_{2}(\cdot, y)\right|_{K}$ is constant within each $K \in \mathcal{T}_{H}$.

LEMMA 5.2 (macroerror). Let $u^{0}$ and $u_{H}^{0}$ be the solutions of problems (3.3) and (5.1), respectively. Assume that (2.2) and (H1) hold and that $u^{0} \in H^{2}\left(\omega_{2}\right)$. Then,

$$
\left\|u^{0}-u_{H}^{0}\right\|_{L^{2}\left(\omega_{2}\right)} \leq C H^{2},
$$

where the constant $C$ is independent of $H, \tilde{h}, h$, and $\varepsilon$, but depends on the stability constant of the bilinear form $B_{2, H}^{0}$.

Proof. For the proof, see [8].

Micro- and modeling errors. For the micro- and modeling errors, we follow [4, sect. 5]. We assume the following regularity on $\psi_{K_{\delta}}^{i}$, the nondiscretized microsolutions of problem (4.2), in $W\left(K_{\delta}\right)$ :

(H2) $\left|\psi_{K_{\delta}}^{i}\right|_{H^{2}\left(K_{\delta}\right)} \leq C \varepsilon^{-1} \sqrt{\left|K_{\delta}\right|}$ for $i=1, \ldots, d$.

To discuss the micro- and modeling errors, we recall that $a_{2}^{0}$ is the homogenized tensor on the domain $\omega_{2}$ and that $a_{2}^{0, h}$ is the numerical homogenized tensor given by (4.3). Consider further the tensor $\bar{a}_{2}^{0}$ defined by (4.3) using the nondiscretized microfunctions $\psi_{K_{\delta}}^{i}$, solutions of (4.2) in $W\left(K_{\delta}\right)$, instead of the discretized functions $\psi_{K_{\delta}}^{i, h}$. The error between the homogenized tensor $a_{2}^{0}$ and its numerical approximation $a_{2}^{0, h}$ can be bounded by

$\sup _{K \in \mathcal{T}_{H}}\left\|a_{2}^{0}\left(x_{K}\right)-a_{2}^{0, h}\left(x_{K}\right)\right\|_{F} \leq \sup _{K \in \mathcal{T}_{H}}\left\|a_{2}^{0}\left(x_{K}\right)-\bar{a}_{2}^{0}\left(x_{K}\right)\right\|_{F}+\sup _{K \in \mathcal{T}_{H}}\left\|\bar{a}_{2}^{0}\left(x_{K}\right)-a_{2}^{0, h}\left(x_{K}\right)\right\|_{F}$,

where the first term on the right-hand side of the above inequality is denoted by $e_{\mathrm{MOD}}$ (modeling error) and the second by $e_{\mathrm{MIC}}$ (microerror).

LEMMA 5.3 (micro- and modeling errors). Let $u_{H}^{0}$ be the solution of (5.1), and let $u^{H}$ be the DG-FE-HMM approximation of $u^{0}$. Assume that (2.2) holds; then

$$
\left.\left\|u_{H}^{0}-u^{H}\right\|\right|_{\omega_{2}} \leq C \sup _{K \in \mathcal{T}_{H}}\left\|a_{2}^{0}\left(x_{K}\right)-a_{2}^{0, h}\left(x_{K}\right)\right\|\left\|_{F}\right\| u^{H} \|
$$

where the constant $C$ is independent of $H, \tilde{h}, h$, and $\varepsilon$. Further, assuming (H2), the Frobenius norm is bounded by

$$
\sup _{K \in \mathcal{T}_{H}}\left\|a_{2}^{0}\left(x_{K}\right)-a_{2}^{0, h}\left(x_{K}\right)\right\|_{F} \leq e_{M O D}+C\left(\frac{h}{\varepsilon}\right)^{2},
$$

where the modeling error $e_{M O D}$ is given in Lemma 5.5.

Proof. The proof follows from [4, sect. 5].

Remark 5.4. A higher order microerror $\left(\frac{h}{\varepsilon}\right)^{2 q}$ can be obtained for higher order micro-FEMs, provided that there is higher order regularity of the microfunctions; i.e.,

$$
\left|\psi_{K_{\delta}}^{i}\right|_{H^{q+1}}\left(K_{\delta}\right) \leq C \varepsilon^{-q} \sqrt{\left|K_{\delta}\right|} \text { for } i=1, \ldots, d .
$$

The modeling error $e_{\mathrm{MOD}}$ will depend on the choice of boundary condition on the microproblems.

Copyright $@$ by SIAM. Unauthorized reproduction of this article is prohibited. 
Lemma 5.5 (modeling error). The modeling error is given by

$$
e_{M O D}= \begin{cases}0, & S^{1}\left(K_{\delta}, \mathcal{T}_{h}\right) \subset W_{p e r}^{1}\left(K_{\delta}\right), \delta / \varepsilon \in \mathbb{N}, \text { and collocation }, \\ C_{1} \delta, & S^{1}\left(K_{\delta}, \mathcal{T}_{h}\right) \subset W_{p e r}^{1}\left(K_{\delta}\right), \delta / \varepsilon \in \mathbb{N}, \\ C_{2} \frac{\varepsilon}{\delta}, & S^{1}\left(K_{\delta}, \mathcal{T}_{h}\right) \subset H_{0}^{1}\left(K_{\delta}\right), \delta / \varepsilon \notin \mathbb{N}, \text { and collocation, } \\ C_{3}\left(\delta+\frac{\varepsilon}{\delta}\right), & S^{1}\left(K_{\delta}, \mathcal{T}_{h}\right) \subset H_{0}^{1}\left(K_{\delta}\right), \delta / \varepsilon \notin \mathbb{N} .\end{cases}
$$

Proof. For the proof, see $[1,2]$.

5.1. A priori error estimates in the fine scale region. In this section, we will prove Theorem 4.3.

Proof of Theorem 4.3. Let $u_{\tilde{h}} \in V_{D}^{1}\left(\omega_{1}, \mathcal{T}_{\tilde{h}}\right)$ be the FE approximation of the physical solution $u^{\varepsilon}$ over the mesh $\mathcal{T}_{\tilde{h}}$, i.e., $u_{\tilde{h}}=u_{1,0, \tilde{h}}+v_{1, \tilde{h}}\left(I^{\tilde{h}} \gamma_{1}(u)\right)$, where $I^{\tilde{h}}$ is the Lagrange interpolant on $\Gamma_{1}$. Classical FE estimates hold:

$$
\left\|u^{\varepsilon}-u_{\tilde{h}}\right\|_{H^{1}\left(\omega^{+}\right)} \leq C \tilde{h}^{s}\left|u^{\varepsilon}\right|_{H^{s+1}\left(\omega_{1}\right)},
$$

where the constant $C$ is independent of $H, h, \tilde{h}$, and $\varepsilon$. Applying a triangular inequality, we obtain

$$
\left\|\nabla\left(u^{\varepsilon}-\bar{u}_{\tilde{h} H}\right)\right\|_{L^{2}\left(\omega^{+}\right)} \leq C \tilde{h}^{s}\left|u^{\varepsilon}\right|_{H^{s+1}\left(\omega_{1}\right)}+\left\|\nabla\left(u_{\tilde{h}}-\bar{u}_{\tilde{h} H}\right)\right\|_{L^{2}\left(\omega^{+}\right)} .
$$

The numerical solution $\bar{u}_{\tilde{h} H}$ over $\omega^{+}$is equal to the numerical fine scale solution $u_{1, \tilde{h}}\left(\theta_{1, \tilde{h}}\right)$, and it holds that

$$
B_{1}\left(u_{\tilde{h}}-u_{1, \tilde{h}}, v_{\tilde{h}}\right)=0 \quad \forall v_{\tilde{h}} \in V_{0}^{1}\left(\omega_{1}, \mathcal{T}_{\tilde{h}}\right),
$$

i.e., the difference $u_{\tilde{h}}-u_{1, \tilde{h}}\left(\theta_{1, \tilde{h}}\right)$ is $a^{\varepsilon}$-harmonic in $\omega_{1}$, and thus the discrete Caccioppoli inequality lemma, Lemma A.5, can be applied. That is,

$$
\left\|\nabla\left(u_{\tilde{h}}-u_{1, \tilde{h}}\left(\theta_{1, \tilde{h}}\right)\right)\right\|_{\mathrm{L}^{2}\left(\omega^{+}\right)} \leq \frac{C}{\left(\tau-\tau^{+}\right)}\left\|u_{\tilde{h}}-u_{1, \tilde{h}}\left(\theta_{1, \tilde{h}}\right)\right\|_{\mathrm{L}^{2}\left(\omega_{1}\right)},
$$

where the constant $C>0$ is independent of $H, \tilde{h}, h$, and $\varepsilon$, but depends on the ellipticity constants of the tensor $a^{\varepsilon}$. Consider an operator $P: V^{1}\left(\Gamma_{1}\right) \times V^{1}\left(\Gamma_{2}\right) \rightarrow$ $V_{D}^{1}\left(\omega_{1}, \mathcal{T}_{\tilde{h}}\right) \times V_{D}^{1}\left(\Omega \backslash \omega_{1}, \mathcal{T}_{H}\right)$ defined as

$$
P\left(\mu_{1, \tilde{h}}, \mu_{2, H}\right)= \begin{cases}u_{1,0, \tilde{h}}+v_{1, \tilde{h}}\left(\mu_{1, \tilde{h}}\right) & \text { in } \omega_{1}, \\ u_{2,0, H}+v_{2, H}\left(\mu_{2, H}\right) & \text { in } \Omega \backslash \omega_{1} .\end{cases}
$$

As in the continuous case, we decompose the operator $P$ as $P=U_{0}+Q$. Over $\omega_{1}$, it holds that $u_{1, \tilde{h}}\left(\theta_{1, \tilde{h}}\right)=P\left(\theta_{1, \tilde{h}}, \theta_{2, H}\right)$ and $u_{\tilde{h}}=P\left(I^{\tilde{h}} \gamma_{1}\left(u^{\varepsilon}\right), I^{H} \gamma_{2}\left(u^{\varepsilon}\right)\right)$. Then,

$$
\begin{aligned}
\left\|u_{\tilde{h}}-u_{1, \tilde{h}}\left(\theta_{1, \tilde{h}}\right)\right\|_{\mathrm{L}^{2}\left(\omega_{1}\right)} & =\left\|P\left(I^{\tilde{h}} \gamma_{1}\left(u^{\varepsilon}\right), I^{H} \gamma_{2}\left(u^{\varepsilon}\right)\right)-P\left(\theta_{1, \tilde{h}}, \theta_{2, H}\right)\right\|_{\mathrm{L}^{2}\left(\omega_{1}\right)} \\
& \leq\|Q\|\left\|\left(I^{\tilde{h}} \gamma_{1}\left(u^{\varepsilon}\right), I^{H} \gamma_{2}\left(u^{\varepsilon}\right)\right)-\left(\theta_{1, \tilde{h}}, \theta_{2, H}\right)\right\|_{\mathrm{L}^{*}(\mathcal{U})} .
\end{aligned}
$$

As $\left(\theta_{1, \tilde{h}}, \theta_{2, H}\right)$ are the discrete optimal virtual controls, they satisfy

$$
\begin{aligned}
& \int_{\omega_{0}}\left(v_{1, \tilde{h}}\left(\theta_{1, \tilde{h}}\right)-v_{2, H}\left(\theta_{2, H}\right)\right)\left(v_{1, \tilde{h}}\left(\mu_{1, \tilde{h}}\right)-v_{2, H}\left(\mu_{2, H}\right)\right) \mathrm{d} x \\
& =-\int_{\omega_{0}}\left(v_{1, \tilde{h}}\left(\mu_{1, \tilde{h}}\right)-v_{2, H}\left(\mu_{2, H}\right)\right)\left(u_{1,0, \tilde{h}}-u_{2,0, H}\right) \mathrm{d} x
\end{aligned}
$$

Copyright (c) by SIAM. Unauthorized reproduction of this article is prohibited. 
for all $\left(\mu_{1, \tilde{h}}, \mu_{2, H}\right) \in V^{1}\left(\Gamma_{1}\right) \times V^{1}\left(\Gamma_{2}\right)$. Then,

$$
\begin{aligned}
& \left\|\left(I^{\tilde{h}} \gamma_{1}\left(u^{\varepsilon}\right), I^{H} \gamma_{2}\left(u^{\varepsilon}\right)\right)-\left(\theta_{1, \tilde{h}}, \theta_{2, H}\right)\right\|_{\mathrm{L}^{*}(\mathcal{U})} \\
& =\sup _{\left(\mu_{1, \tilde{h}}, \mu_{2, H}\right)} \frac{\left|\pi\left(\left(I^{\tilde{h}} \gamma_{1}\left(u^{\varepsilon}\right), I^{H} \gamma_{2}\left(u^{\varepsilon}\right)\right),\left(\mu_{1, \tilde{h}}, \mu_{2, H}\right)\right)-\pi\left(\left(\theta_{1, \tilde{h}}, \theta_{2, H}\right),\left(\mu_{1, \tilde{h}}, \mu_{2, H}\right)\right)\right|}{\left\|\left(\mu_{1, \tilde{h}}, \mu_{2, H}\right)\right\|_{\mathrm{L}^{*}(\mathcal{U})}},
\end{aligned}
$$

and following the proof of Lemma 3.3,

$$
\begin{aligned}
\pi\left(\left(I^{\tilde{h}} \gamma_{1}\left(u^{\varepsilon}\right), I^{H} \gamma_{2}\left(u^{\varepsilon}\right)\right),\left(\mu_{1, \tilde{h}}, \mu_{2, H}\right)\right) & -\pi\left(\left(\theta_{1, \tilde{h}}, \theta_{2, H}\right),\left(\mu_{1, \tilde{h}}, \mu_{2, H}\right)\right) \\
& =\int_{\omega_{0}}\left(u_{\tilde{h}}-u^{H}\right)\left(v_{1, \tilde{h}}\left(\mu_{1, \tilde{h}}\right)-v_{2, H}\left(\mu_{2, H}\right)\right) \mathrm{d} x \\
& \leq\left\|u_{\tilde{h}}-u^{H}\right\|_{\mathrm{L}^{2}\left(\omega_{0}\right)}\left\|\left(\mu_{1, \tilde{h}}, \mu_{2, H}\right)\right\|_{\mathrm{L}^{*}(\mathcal{U})},
\end{aligned}
$$

where $u^{H}=u_{2,0, H}+v_{2, H}\left(I^{H} \gamma_{2}\left(u^{\varepsilon}\right)\right)$. We obtain that

$$
\left\|\left(I^{\tilde{h}} \gamma_{1}\left(u^{\varepsilon}\right), I^{H} \gamma_{2}\left(u^{\varepsilon}\right)\right)-\left(\theta_{1, \tilde{h}}, \theta_{2, H}\right)\right\|_{\mathrm{L}^{*}(\mathcal{U})} \leq\left\|u_{\tilde{h}}-u^{H}\right\|_{\mathrm{L}^{2}\left(\omega_{0}\right)},
$$

and summarizing, we have

$$
\left\|\nabla\left(u_{\tilde{h}}-u_{1, \tilde{h}}\left(\theta_{1, \tilde{h}}\right)\right)\right\|_{\mathrm{L}^{2}\left(\omega^{+}\right)} \leq C\left\|u_{\tilde{h}}-u^{H}\right\|_{\mathrm{L}^{2}\left(\omega_{0}\right)} .
$$

Then, we decompose the error into

$$
\left\|u_{\tilde{h}}-u^{H}\right\|_{\mathrm{L}^{2}\left(\omega_{0}\right)} \leq\left\|u_{\tilde{h}}-u^{\varepsilon}\right\|_{\mathrm{L}^{2}\left(\omega_{0}\right)}+\left\|u^{\varepsilon}-u^{0}\right\|_{\mathrm{L}^{2}\left(\omega_{0}\right)}+\left\|u^{0}-u^{H}\right\|_{\mathrm{L}^{2}\left(\omega_{0}\right)},
$$

and provided that the solutions $u^{\varepsilon}$ and $u^{0}$ are smooth enough, standard FE estimates and (3.4) can be applied to bound the first two quantities in (5.3), i.e.,

$$
\left\|u_{\tilde{h}}-u^{H}\right\|_{L^{2}\left(\omega_{0}\right)} \leq C \tilde{h}^{s+1}\left|u^{\varepsilon}\right|_{H^{s+1}\left(\omega_{1}\right)}+C \varepsilon+\left\|u^{0}-u^{H}\right\|_{L^{2}\left(\omega_{0}\right)} .
$$

We bound the error in $\omega_{0}$ by the error in $\omega_{2}$ :

$$
\left\|u^{0}-u^{H}\right\|_{\mathrm{L}^{2}\left(\omega_{0}\right)} \leq\left\|u^{0}-u^{H}\right\|_{\mathrm{L}^{2}\left(\omega_{2}\right)} \leq\left\|u^{0}-u_{H}^{0}\right\|_{\mathrm{L}^{2}\left(\omega_{2}\right)}+\left\|u_{H}^{0}-u^{H}\right\|_{\mathrm{L}^{2}\left(\omega_{2}\right)} .
$$

The two norms correspond to the DG-FE-HMM error in the $L^{2}$ norm and are given by Lemmas $5.2,5.3$, and 5.5.

5.2. A priori error estimates in the scale separated region. We prove an a priori error bound between $u^{\varepsilon}$ and $\bar{u}_{\tilde{h} H}$ in $\Omega \backslash \omega^{+}$, where $\bar{u}_{\tilde{h} H}$ is defined in (4.14). For simplicity, we assume that $\delta=\varepsilon$ and choose periodic coupling conditions between the macro- and the microproblem. We recall that the reconstructed homogenized solution $u_{2}^{r e c}$, and its numerical approximation $u_{2, H}^{r e c}$, are given by

$$
\begin{aligned}
& u_{2}^{r e c}(x)=u_{2}^{0}(x)+\varepsilon \sum_{j=1}^{d} \chi^{j}\left(x, \frac{x}{\varepsilon}\right) \frac{\partial u_{2}^{0}(x)}{\partial x_{j}}, \\
& u_{2, H}^{r e c}(x)=u_{2, H}(x)+\sum_{j=1}^{d} \psi_{K_{\varepsilon}}^{j, h}(x) \frac{\partial u_{2, H}(x)}{\partial x_{j}},
\end{aligned}
$$

where $u_{2}^{0}=u_{2}^{0}\left(\theta_{2}\right)$ and $u_{2, H}=u_{2, H}\left(\theta_{2, H}\right)$ are the exact solution and the numerical solution of the coupling in $\omega_{2}$, respectively, and $\psi_{K_{\varepsilon}}^{j, h}$ are the microsolutions of (4.2). 
We sometimes use $u_{2}^{r e c}\left(\theta_{2}\right)$ and $u_{2, H}^{r e c}\left(\theta_{2, H}\right)$ to emphasize the dependence on $\theta_{2}$ and $\theta_{2, H}$, respectively.

We introduce the discrete microproblems on $K_{\varepsilon}$ : find $u^{h}$ such that $u^{h}-u_{2, H} \in$ $S^{1}\left(K_{\varepsilon}, \mathcal{T}_{h}\right)$ and

$$
\int_{K_{\varepsilon}} a_{2}^{\varepsilon}(x) \nabla v^{h} \cdot \nabla z^{h} \mathrm{~d} x=0 \quad \forall z^{h} \in S^{1}\left(K_{\varepsilon}, \mathcal{T}_{h}\right) .
$$

From assumption (H1), the tensor $a_{2}^{\varepsilon}$ is constant in each macroelement $K \in \mathcal{T}_{H}$. This simplifies the analysis, as the modeling error is zero. We introduce a semidiscrete problem over $\omega_{2}$ : find $\bar{u}_{2, H} \in V_{D}^{1}\left(\omega_{2}, \mathcal{T}_{H}\right)$, the solution of

$$
\begin{aligned}
\bar{B}_{2, H}\left(\bar{u}_{2, H}, w_{H}\right) & =F_{2}\left(w_{H}\right) \quad \forall w_{H} \in V^{1}\left(\omega_{2}, \mathcal{T}_{H}\right), \\
\bar{u}_{2, H} & =\theta_{2, H} \quad \text { on } \Gamma_{2},
\end{aligned}
$$

where the bilinear form $\bar{B}_{2, H}$ on $V^{1}\left(\omega_{2}, \mathcal{T}_{H}\right) \times V^{1}\left(\omega_{2}, \mathcal{T}_{H}\right)$ is given by

$$
\begin{aligned}
& \bar{B}_{2, H}\left(v_{H}, w_{H}\right)=\sum_{K \in \mathcal{T}_{H}} \frac{|K|}{\left|K_{\varepsilon}\right|} \int_{K_{\varepsilon}} a_{2}^{\varepsilon}(x) \nabla v \cdot \nabla w \mathrm{~d} x+\sum_{e \in E} \int_{e} \mu_{e} \llbracket v_{H} \rrbracket \llbracket w_{H} \rrbracket \mathrm{d} s \\
& -\sum_{e \in E} \int_{e}\left(\overline{\left\{a_{2}^{\varepsilon} \nabla v\right\}} \llbracket w_{H} \rrbracket+\overline{\left\{a_{2}^{\varepsilon} \nabla w\right\}} \llbracket v_{H} \rrbracket\right) \mathrm{d} s,
\end{aligned}
$$

where $v$ and $w$ are solutions of (5.6) in the exact Sobolev space $W\left(K_{\varepsilon}\right)$.

For a vector valued function $\eta$, we define the average of the multiscale fluxes as

$$
\overline{\{\eta\}}=\frac{1}{2}\left(\frac{1}{\left|K_{\varepsilon}^{+}\right|} \int_{K_{\varepsilon}^{+}} \eta_{+} \mathrm{d} x+\frac{1}{\left|K_{\varepsilon}^{-}\right|} \int_{K_{\varepsilon}^{-}} \eta_{-} \mathrm{d} x\right) .
$$

We can then define $\bar{u}_{2, H}^{r e c}$ by

$$
\bar{u}_{2, H}^{r e c}(x)=\bar{u}_{2, H}(x)+\sum_{j=1}^{d} \psi_{K_{\varepsilon}}^{j}(x) \frac{\partial \bar{u}_{2, H}(x)}{\partial x_{j}}, \quad x \in K,
$$

where $\bar{u}_{2, H}=\bar{u}_{2, H}\left(\theta_{2, H}\right)$. We use $\bar{u}_{2, H}^{r e c}\left(\theta_{2, H}\right)$ to denote the dependence on $\theta_{2, H}$.

We now give the proof of Theorem 4.4.

Proof of Theorem 4.4. We decompose the error into

$\left\|u^{\varepsilon}-u_{2, H}^{r e c}\left(\theta_{2, H}\right)\right\|_{\overline{\mathrm{H}}^{1}\left(\Omega \backslash \omega^{+}\right)} \leq\left\|u^{\varepsilon}-u_{2}^{r e c}\left(\theta_{2}\right)\right\|_{\overline{\mathrm{H}}^{1}\left(\Omega \backslash \omega^{+}\right)}+\left\|u_{2}^{r e c}\left(\theta_{2}\right)-u_{2, H}^{r e c}\left(\theta_{2, H}\right)\right\|_{\overline{\mathrm{H}}^{1}\left(\Omega \backslash \omega^{+}\right)}$.

From Theorem 3.6, it holds that $\left\|u^{\varepsilon}-u_{2}^{r e c}\left(\theta_{2}\right)\right\|_{\overline{\mathrm{H}}^{1}\left(\Omega \backslash \omega^{+}\right)} \leq C_{1} \varepsilon^{1 / 2}$. We focus on $\left\|u_{2}^{r e c}\left(\theta_{2}\right)-u_{2, H}^{r e c}\left(\theta_{2, H}\right)\right\|_{\overline{\mathrm{H}}^{1}\left(\Omega \backslash \omega^{+}\right)}$and follow [2, sect. 3.3.3]. Using the triangular inequality, we obtain

$$
\begin{aligned}
\left\|u^{\varepsilon}-u_{2, H}^{r e c}\left(\theta_{2, H}\right)\right\|_{\overline{\mathrm{H}}^{1}\left(\Omega \backslash \omega^{+}\right)} \leq & C_{1} \varepsilon^{1 / 2}+\left\|u_{2}^{r e c}\left(\theta_{2}\right)-u_{2, H}^{r e c}\left(\theta_{2, H}\right)\right\|_{\overline{\mathrm{H}}^{1}\left(\Omega \backslash \omega^{+}\right)} \\
\leq & C_{1} \varepsilon^{1 / 2}+\left\|u_{2}^{r e c}\left(\theta_{2}\right)-\bar{u}_{2, H}^{\mathrm{rec}}\left(\theta_{2, H}\right)\right\|_{\overline{\mathrm{H}}^{1}\left(\Omega \backslash \omega^{+}\right)} \\
& +\left\|\bar{u}_{2, H}^{\mathrm{rec}}\left(\theta_{2, H}\right)-u_{2, H}^{r e c}\left(\theta_{2, H}\right)\right\|_{\overline{\mathrm{H}}^{1}\left(\Omega \backslash \omega^{+}\right)} .
\end{aligned}
$$

Lemma 5.7 gives us

$$
\begin{aligned}
\left\|u_{2}^{r e c}\left(\theta_{2}\right)-\bar{u}_{2, H}^{\mathrm{rec}}\left(\theta_{2, H}\right)\right\|_{\overline{\mathrm{H}}^{1}\left(\Omega \backslash \omega^{+}\right)} \leq & C_{3} H\left|u_{2}^{0}\right|_{H^{2}\left(\omega_{2}\right)} \\
& +\frac{C_{4}}{\tau^{+}}\left(\tilde{h}^{s+1}\left|u_{1}^{\varepsilon}\right|_{H^{s+1}\left(\omega_{1}\right)}+\varepsilon+H^{2}\left|u_{2}^{0}\right|_{H^{2}\left(\omega_{2}\right)}\right) .
\end{aligned}
$$

Copyright $@$ by SIAM. Unauthorized reproduction of this article is prohibited. 
Further, Lemma 5.9 provides us with

$$
\left\|\bar{u}_{2, H}^{\mathrm{rec}}\left(\theta_{2, H}\right)-u_{2, H}^{r e c}\left(\theta_{2, H}\right)\right\|_{\overline{\mathrm{H}}^{1}\left(\Omega \backslash \omega^{+}\right)} \leq C_{2}\left(\frac{h}{\varepsilon}\right) .
$$

Collecting the previous results gives

$$
\begin{aligned}
\left\|u_{2}^{r e c}\left(\theta_{2}\right)-u_{2, H}^{r e c}\left(\theta_{2, H}\right)\right\|_{\overline{\mathrm{H}}^{1}\left(\Omega \backslash \omega^{+}\right)} \leq & C_{1} \varepsilon^{1 / 2}+C_{2}\left(\frac{h}{\varepsilon}\right)+C_{3} H\left|u_{2}^{0}\right|_{H^{2}\left(\omega_{2}\right)} \\
& +\frac{C_{4}}{\tau^{+}}\left(\tilde{h}^{s+1}\left|u_{1}^{\varepsilon}\right|_{H^{s+1}\left(\omega_{1}\right)}+\varepsilon+H^{2}\left|u_{2}^{0}\right|_{H^{2}\left(\omega_{2}\right)}\right) .
\end{aligned}
$$

Remark 5.6. Theorem 4.4 can be adapted for the general tensor $a_{2}^{\varepsilon}(x)$ without a two-scale structure. In that case, the modeling error is present in the last term of the error.

Recall that we assume periodic coupling with $\delta=\varepsilon$ and that (H1) and (H2) hold. Further, we assume Lipschitz continuity of the tensor in the first variable, i.e., $a_{2}(x, y) \in W^{1, \infty}\left(\bar{\omega}_{2}, L^{\infty}(Y)\right)$.

Lemma 5.7. Let $u_{2}^{\text {rec }}\left(\theta_{2}\right)$ and $\bar{u}_{2, H}^{\text {rec }}\left(\theta_{2, H}\right)$ be given by (5.4) and (5.7). Assume that $u_{2}^{0} \in H^{2}\left(\omega_{2}\right), u_{1}^{\varepsilon} \in H^{s+1}\left(\omega_{1}\right)$, with $s \leq 1$, and that the exact solutions of the microproblem (4.2) verify $(\mathrm{H} 2)$. Then

$$
\begin{aligned}
\left\|u_{2}^{r e c}\left(\theta_{2}\right)-\bar{u}_{2, H}^{r e c}\left(\theta_{2, H}\right)\right\|_{\overline{\mathrm{H}}^{1}\left(\Omega \backslash \omega^{+}\right)} \leq & C_{1} H\left|u_{2}^{0}\right|_{H^{2}\left(\omega_{2}\right)} \\
& +\frac{C_{2}}{\tau^{+}}\left(\tilde{h}^{s+1}\left|u_{1}^{\varepsilon}\right|_{H^{s+1}\left(\omega_{1}\right)}+\varepsilon+H^{2}\left|u_{2}^{0}\right|_{H^{2}\left(\omega_{2}\right)}\right),
\end{aligned}
$$

where the constants are independent of $H, \tilde{h}, h$, and $\varepsilon$.

Proof. Using the definitions of $u_{2}^{r e c}\left(\theta_{2}\right)$ and $\bar{u}_{2, H}^{r e c}\left(\theta_{2, H}\right)$, it holds that

$$
\begin{aligned}
& \left\|u_{2}^{r e c}\left(\theta_{2}\right)-\bar{u}_{2, H}^{r e c}\left(\theta_{2, H}\right)\right\|_{\overline{\mathrm{H}}^{1}\left(\Omega \backslash \omega^{+}\right)}^{2}=\sum_{K \in \mathcal{T}_{H}\left(\Omega \backslash \omega^{+}\right)}\left\|\nabla\left(u_{2}^{r e c}\left(\theta_{2}\right)-\bar{u}_{2, H}^{r e c}\left(\theta_{2, H}\right)\right)\right\|_{\mathrm{L}^{2}(K)}^{2} \\
& \leq \sum_{K \in \mathcal{T}_{H}\left(\Omega \backslash \omega^{+}\right)}\left\|\nabla\left(u_{2}^{0}-\bar{u}_{2, H}\right)\right\|_{\mathrm{L}^{2}(K)}^{2} \\
& \quad+\sum_{K \in \mathcal{T}_{H}\left(\Omega \backslash \omega^{+}\right)}\left\|\sum_{j=1}^{d} \nabla\left(\varepsilon \chi^{j}\left(x, \frac{x}{\varepsilon}\right) \frac{\partial u_{2}^{0}}{\partial x_{j}}-\psi_{K_{\varepsilon}}^{j}(x) \frac{\partial \bar{u}_{2, H}}{\partial x_{j}}\right)\right\|_{L^{2}(K)}^{2} .
\end{aligned}
$$

Thanks to (H1), it holds that $\varepsilon \chi^{j}(x, x / \varepsilon)=\psi_{K_{\varepsilon}}^{j}(x)$, and the second norm is bounded by the first norm. We recall the bilinear form (5.2) for problem (2.3) with a quadrature formula,

$$
\begin{aligned}
B_{2, H}^{0}\left(v_{H}, w_{H}\right)= & \sum_{K \in \mathcal{T}_{H}}|K| a_{2}^{0}\left(x_{K}\right) \nabla v_{H} \cdot \nabla w_{H}+\sum_{e \in E} \int_{e} \mu_{e} \llbracket v_{H} \rrbracket \llbracket w_{H} \rrbracket \mathrm{d} s \\
& -\sum_{e \in E} \int_{e}\left(\left\{a_{2}^{0}\left(x_{K}, x / \varepsilon\right) v\right\} \llbracket w_{H} \rrbracket+\left\{a_{2}^{0}\left(x_{K}, x / \varepsilon\right) w\right\} \llbracket v_{H} \rrbracket\right) \mathrm{d} s,
\end{aligned}
$$

and define $\hat{u}_{2, H}\left(\theta_{2, H}\right) \in V_{D}^{1}\left(\omega_{2}, \mathcal{T}_{H}\right)$, the solution of

$$
B_{2, H}^{0}\left(\hat{u}_{2, H}, w_{H}\right)=F_{2}\left(w_{H}\right) \quad \forall w_{H} \in V_{0}^{1}\left(\omega_{2}, \mathcal{T}_{H}\right) .
$$

Copyright $@$ by SIAM. Unauthorized reproduction of this article is prohibited. 
By $\left[2\right.$, Proposition 14], it holds that $\bar{u}_{2, H}=\hat{u}_{2, H}$. By hypothesis, $u_{2}^{0}\left(\theta_{2}\right)$ and $\bar{u}_{2, H}\left(\theta_{2, H}\right)$ have zero boundary conditions on $\partial \omega_{2} \cap \partial \Omega$, and we can use [13, Lemmas 4.1 and 4.2 ] to obtain

$$
\begin{aligned}
\left\|u_{2}^{0}\left(\theta_{2}\right)-\bar{u}_{2, H}\left(\theta_{2, H}\right)\right\|_{\overline{\mathrm{H}}^{1}\left(\Omega \backslash \omega^{+}\right)} \leq & C_{1} \inf _{w \in V_{D}^{1}\left(\omega_{2}, \mathcal{T}_{H}\right), w=I^{H} \theta_{2} \text { on } \Gamma_{2}}\left\|u_{2}^{0}\left(\theta_{2}\right)-w\right\|_{\overline{\mathrm{H}}^{1}\left(\omega_{2}\right)} \\
& +\frac{C_{2}}{\tau^{+}}\left\|u_{2}^{0}\left(\theta_{2}\right)-\bar{u}_{2, H}\left(\theta_{2, H}\right)\right\|_{\mathrm{L}^{2}\left(\omega_{2}\right)} .
\end{aligned}
$$

The first norm can be bounded by

$$
\inf _{w}\left\|u_{2}^{0}\left(\theta_{2}\right)-w\right\|_{\overline{\mathrm{H}}^{1}\left(\omega_{2}\right)} \leq\left\|u_{2}^{0}\left(\theta_{2}\right)-u_{2, H}\left(I^{H} \theta_{2}\right)\right\|_{\overline{\mathrm{H}}^{1}\left(\omega_{2}\right)} \leq C_{1} H\left|u_{2}^{0}\right|_{H^{2}\left(\omega_{2}\right)},
$$

where $u_{2, H}\left(I^{H} \theta_{2}\right)$ is the FEM solution with an interpolation of $\theta_{2}$ on $\Gamma_{2}$. Following the proof of Theorem 4.3, the second part is bounded by

$$
\begin{aligned}
\left\|u_{2}^{0}\left(\theta_{2}\right)-\bar{u}_{2, H}\left(\theta_{2, H}\right)\right\|_{\mathrm{L}^{2}\left(\omega_{2}\right)} \leq & \left\|u_{2}^{0}\left(\theta_{2}\right)-u_{2, H}\left(I^{H} \theta_{2}\right)\right\|_{\mathrm{L}^{2}\left(\omega_{2}\right)} \\
& +\left\|u_{2, H}\left(I^{H} \theta_{2}\right)-\bar{u}_{2, H}\left(\theta_{2, H}\right)\right\|_{\mathrm{L}^{2}\left(\omega_{2}\right)} \\
\leq & C_{1} H^{2}\left|u_{2}^{0}\right|_{H^{2}\left(\omega_{2}\right)}+\left\|Q\left(I^{\tilde{h}} \theta_{1}, I^{H} \theta_{2}\right)-Q\left(\theta_{1, \tilde{h}}, \theta_{2, H}\right)\right\|_{\mathrm{L}^{2}\left(\omega_{2}\right)} \\
\leq & C_{1} H^{2}\left|u_{2}^{0}\right|_{H^{2}\left(\omega_{2}\right)}+C_{2}\left\|u_{1, \tilde{h}}\left(I^{\tilde{h}} \theta_{1}\right)-\bar{u}_{2, H}\left(I^{H} \theta_{2}\right)\right\|_{\mathrm{L}^{2}\left(\omega_{0}\right)},
\end{aligned}
$$

where we have used that $\left(\theta_{1}^{\tilde{h}}, \theta_{2}^{H}\right)$ is the optimal couple of the discrete minimization problem and that $Q$ is bounded. Finally, using the triangular inequality, we have

$$
\begin{aligned}
\left\|u_{1, \tilde{h}}\left(I^{\tilde{h}} \theta_{1}\right)-\bar{u}_{2, H}\left(I^{H} \theta_{2}\right)\right\|_{\mathrm{L}^{2}\left(\omega_{0}\right)} \leq & \left\|u_{1, \tilde{h}}\left(I^{\tilde{h}} \theta_{1}\right)-u_{1}^{\varepsilon}\left(\theta_{1}\right)\right\|_{\mathrm{L}^{2}\left(\omega_{0}\right)} \\
& +\left\|u_{1}^{\varepsilon}\left(\theta_{1}\right)-u_{2}^{0}\left(\theta_{2}\right)\right\|_{\mathrm{L}^{2}\left(\omega_{0}\right)} \\
& +\left\|u_{2}^{0}\left(\theta_{2}\right)-\bar{u}_{2, H}\left(I^{H} \theta_{2}\right)\right\|_{\mathrm{L}^{2}\left(\omega_{0}\right)} \\
\leq & C\left(\tilde{h}^{s+1}\left|u_{1}^{\varepsilon}\right|_{H^{s+1}\left(\omega_{1}\right)}+\varepsilon+H^{2}\left|u_{2}^{0}\right|_{H^{2}\left(\omega_{2}\right)}\right) .
\end{aligned}
$$

Summarizing,

$$
\begin{aligned}
\left\|u_{2}^{r e c}\left(\theta_{2}\right)-\bar{u}_{2, H}^{r e c}\left(\theta_{2, H}\right)\right\|_{\bar{H}^{1}\left(\Omega \backslash \omega^{+}\right)} \leq & C_{1} H\left|u_{2}^{0}\right|_{H^{2}\left(\omega_{2}\right)} \\
& +\frac{C_{2}}{\tau^{+}}\left(\tilde{h}^{s+1}\left|u_{1}^{\varepsilon}\right|_{H^{s+1}\left(\omega_{1}\right)}+\varepsilon+H^{2}\left|u_{2}^{0}\right|_{H^{2}\left(\omega_{2}\right)}\right) .
\end{aligned}
$$

The result of the lemma follows.

Remark 5.8. The proof of Lemma 5.7 can be generalized for functions with nonhomogeneous boundary conditions. This can be done by splitting the solutions into a function depending on the controls and a function independent of the controls. The proof follows the same lines.

Lemma 5.9. Let $\bar{u}_{2, H}^{r e c}\left(\theta_{2, H}\right)$ and $u_{2, H}^{r e c}\left(\theta_{2, H}\right)$ be defined by (5.7) and (5.5), respectively. Then

$$
\left\|\bar{u}_{2, H}^{r e c}\left(\theta_{2, H}\right)-u_{2, H}^{r e c}\left(\theta_{2, H}\right)\right\|_{\overline{\mathrm{H}}^{1}\left(\Omega \backslash \omega^{+}\right)} \leq C\left(\frac{h}{\varepsilon}\right) .
$$

Proof. The proof follows from [2, sect. 3.3.3].

Copyright (C) by SIAM. Unauthorized reproduction of this article is prohibited. 
6. Numerical experiments. In this section we present various numerical experiments to illustrate the convergence rates and the performance of our method. In the first two examples, we compare our coupling method with the classical global-local method [36], where the homogenized solution is considered as the boundary condition on $\Gamma_{1}$. To facilitate the numerical comparison, we assume that the meshes $\mathcal{T}_{\tilde{h}}$ and $\mathcal{T}_{H}$ have the same triangulation in the overlap $\omega_{0}$. The implementations can be adapted to the case where the meshes are not equal in $\omega_{0}$, using interpolations between the two meshes.

Computational costs of the methods. Here, we briefly comment on the computational cost of the optimization based method and of the global-local method [36]. Both methods use the FE-HMM (or DG-FE-HMM) and the FEM.

Let $N$ denote the total degrees of freedom (DOF) of the initial triangulation over $\Omega$, and let $N_{m i c}$ denote the micro-DOF needed to obtain the homogenized conductivity at the quadrature points of the macromesh. Further, let $N_{\omega_{1}}$ be the DOF of the fine triangulation in $\omega_{1}$, and let $N_{\Omega \backslash \omega_{1}}, N_{\omega_{2}}$ be the DOF of the coarse triangulation over $\Omega \backslash \omega_{1}$ and $\omega_{2}=\Omega \backslash \omega$, respectively.

For the classical global-local method, (DG-)FE-HMM provides us with a numerical homogenized solution $u^{H}$, which is used as the boundary condition on $\Gamma_{1}$, and the total cost is $\mathcal{O}\left(N \cdot N_{\text {mic }}\right)+\mathcal{O}\left(N_{\omega_{1}}\right)$.

For the optimization based method, we start by computing the numerical solutions $u_{1,0, \tilde{h}}$ and $u_{2,0, H}$, using FEM and (DG-)FE-HMM, respectively. The cost is $\mathcal{O}\left(N_{\omega_{1}}\right)$ for FEM and $\mathcal{O}\left(N_{\omega_{2}} \cdot N_{m i c}\right)$ for (DG-)FE-HMM. Then, we solve a saddle point problem with cost $\mathcal{O}\left(N_{\omega_{1}}+N_{\Omega \backslash \omega_{1}}\right)$. We note that the cost of the optimization based method can be further reduced [6].

6.1. A domain with a crack. Consider an elliptic boundary value problem in $\Omega=[0,1]^{2}$,

$$
-\operatorname{div}\left(a^{\varepsilon} \nabla u\right)=0 \quad \text { in } \Omega,
$$

with Dirichlet boundary condition $u=\varphi$ on $\partial \Omega$, where $\varphi \in[0,2 \pi]$ is the angle measured counterclockwise from the axis $\{(x, 0.5): x \geq 0\}$. We add a free Neumann boundary condition on the crack $\left\{x \in \Omega: x_{1} \geq 0, x_{2}=0.5\right\}$. The homogenization model might not be accurate around the crack. A mesh refinement of the coarse model around the crack may lead to coarse meshes with mesh size smaller than $\varepsilon$; hence it requires more work around the crack than the FEM with scale resolution. For the treatment of the crack problem with the FE-HMM, we refer the reader to [7]. We take a tensor $a^{\varepsilon}$-represented in Figure 2(a) for $\varepsilon=1 / 10$ - with separation of scale and locally periodic in $Y$,

$$
a^{\varepsilon}\left(x_{1}, x_{2}\right)=\left(\frac{1}{\left(1.1+\cos \left(2 \pi \frac{x_{1}}{\varepsilon}\right)\right)^{2}}+\frac{1}{\left(1.1+\cos \left(2 \pi \frac{x_{2}}{\varepsilon}\right)\right)^{2}}\right)^{1 / 2} .
$$

Let $x_{c}=[1 / 2,1 / 2]$ be the center of $\Omega$, and let $\omega_{1}=x_{c}+\frac{1}{15}[-1,1]^{2}$ and $\Gamma_{1}=\partial \omega_{1}$. The classical global-local numerical solution is the approximation of the following problem:

$$
\begin{aligned}
-\operatorname{div}\left(a^{\varepsilon} \nabla u\right) & =f & & \text { in } \omega_{1}, \\
u & =u^{0} & & \text { on } \Gamma_{1},
\end{aligned}
$$

where $u^{0}$ is the homogenized solution. Recall that $\omega \subset \omega_{1}$, where $\omega=x_{c}+\frac{1}{30}[-1,1]^{2}$. We compute the numerical homogenized solution $u^{H}$ over $\Omega$ on the coarse initial mesh 


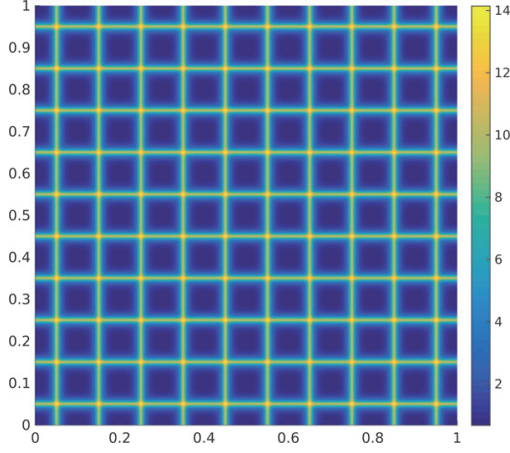

(a)

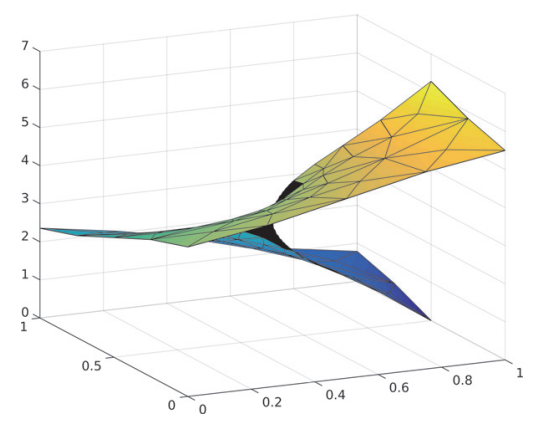

(c)

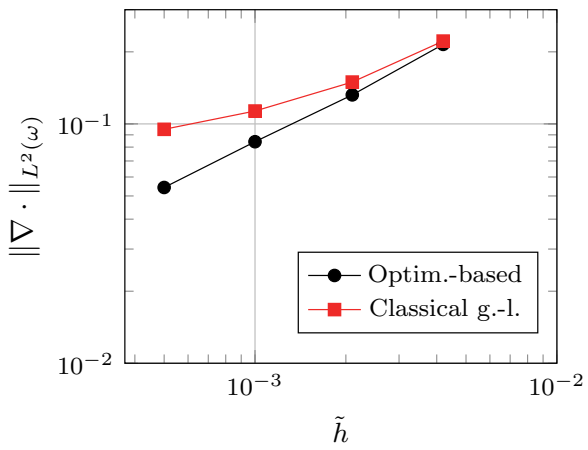

(b)

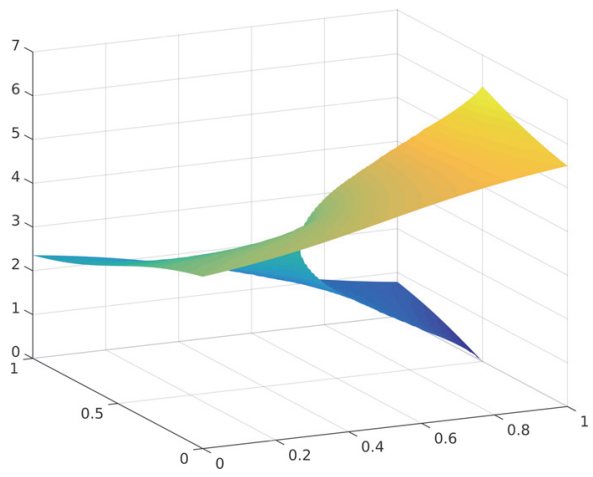

(d)

FIG. 2. Crack experiment: (a) tensor for $\varepsilon=1 / 10$, (b) $H^{1}$ seminorm in $\omega$ for the optimization based coupling (black) and the classical coupling (red) (color available online), (c) numerical optimization based solution, and (d) reference solution.

and use the value of $u^{H}$ as Dirichlet boundary condition on $\Gamma_{1}$ to solve problem (6.1) with a fine scale FEM.

We refine uniformly in $\omega_{1}$, and as the mesh size in $\omega$ should be small enough to capture the microscopic scales of the problems, it would be prohibitive to compute the numerical homogenized solution at each iteration. The coupling and the classical global-local method are both performed on the same mesh, while the coarse mesh in $\Omega \backslash \omega_{1}$ is left unchanged. We then compare the numerical solution with a reference solution obtained with an FEM on a very fine mesh. The reference solution is shown in Figure 2(d) and the numerical optimization based coupling solution in Figure 2(c). We plot the $H^{1}$ seminorm for the two methods in Figure 2(b). We see that the globallocal method reaches a threshold value, as expected due to the use of the numerical homogenized function $u^{H}$ as Dirichlet data on $\Gamma_{1}$.

6.2. Singular source term. In this experiment, we consider an elliptic problem with a singular source term given by random peaks. The tensor is assumed to have scale separation and is given by

$$
a^{\varepsilon}(x)=\frac{1}{6}\left(\frac{1.1+\sin \left(2 \pi \frac{x_{1}}{\varepsilon} \frac{x_{2}}{\varepsilon}\right)}{1.1+\sin \left(2 \pi \frac{x_{2}}{\varepsilon}\right)}+\sin \left(4 x_{1}^{2} x_{2}^{2}\right)+2\right) .
$$




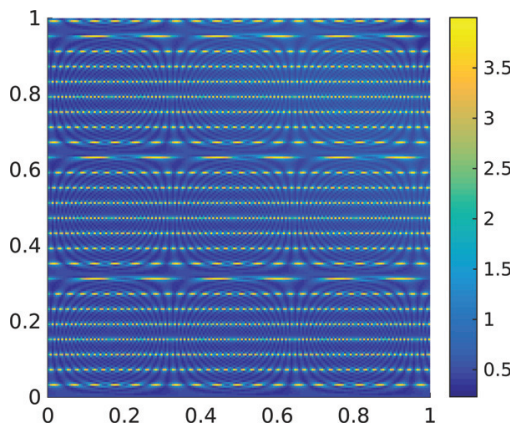

(a)

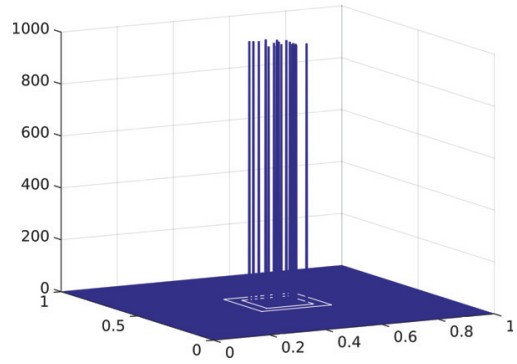

(b)

FIG. 3. Singular source term experiment: (a) tensor for $\varepsilon=1 / 25$, (b) right-hand side with 20 random peaks.

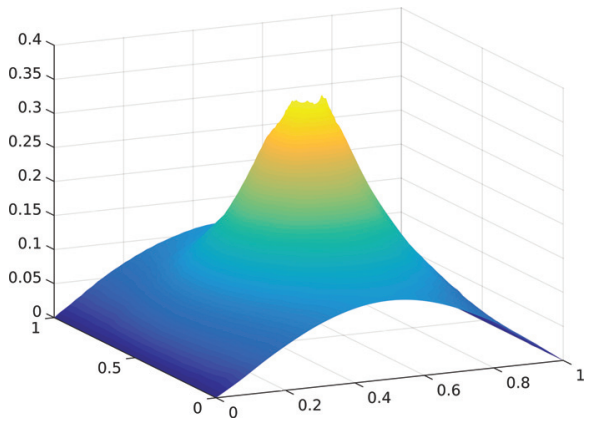

(a)

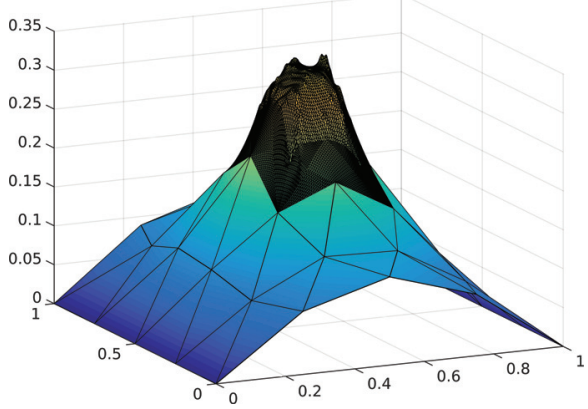

(b)

FIG. 4. Singular source term experiment: (a) reference solution, (b) optimization based numerical solution.

Depending on the location of the random peaks, the numerical homogenized righthand side $f^{0}$ can be wrong, leading to an inaccurate approximation of $u^{0}$. As in the crack experiments, we compute a numerical approximation of $u^{0}$ on a coarse initial mesh and then use it as boundary condition on $\Gamma_{1}$. In Figure $3(\mathrm{a})$ we show the tensor for $\varepsilon=1 / 25$. Let $x_{c}=[1 / 2,1 / 2]$ be the center of $\Omega$; we set $\omega=x_{c}+\frac{1}{12}[-1,1]^{2}$ and $\omega_{1}=x_{c}+\frac{1}{4}[-1,1]^{2}$. In Figure $3(\mathrm{~b})$, we illustrate the random source term $f$ with 20 peaks. Figures $4(\mathrm{a})$ and $4(\mathrm{~b})$ illustrate the reference solution and the optimization based solutions, with the fine scale solution in $\omega$ and the coarse scale solution in $\Omega \backslash \omega$. The $H^{1}$ error between the numerical and reference solutions, for $\varepsilon=1 / 10$ and 100 random peaks, is shown in Figure 5 for the classical global-local method (in red) and the coupling (in black) (color available online). While we observe a linear convergence rate for the optimization based method, as predicted by Theorem 4.3, we see that the classical coupling leads to saturation in the error decay. This is due to inaccurate boundary conditions for the fine scale problems.

6.3. A domain with a defect. We consider a homogenization problem with a local perturbation in the tensor, treated in [11]. The PDE is

$$
\begin{aligned}
-\operatorname{div}\left(a^{\varepsilon} \nabla u^{\varepsilon}\right) & =f & & \text { in } \Omega, \\
u^{\varepsilon} & =0 & & \text { on } \partial \Omega,
\end{aligned}
$$




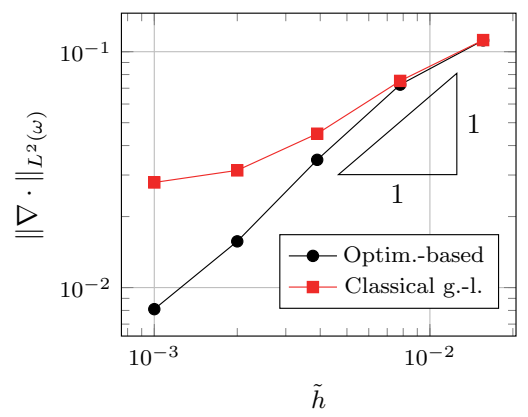

FIG. 5. Singular source term experiment: $H^{1}$ seminorm in $\omega$ for the optimization based coupling (black) and the classical coupling (red) (color available online).

where the tensor is of the form $a^{\varepsilon}=a_{p e r}^{\varepsilon}(x)+b^{\varepsilon}(x)$, where $a_{\text {per }}^{\varepsilon}(x)=a_{\text {per }}(x, x / \varepsilon)$ is (locally) periodic and $b^{\varepsilon} \in L^{2}(\Omega)^{2}$ is a local perturbation of size $\varepsilon$. A numerical homogenized solution $u^{H}$ can be obtained with FE-HMM and produces a good approximation of $u^{\varepsilon}$ in the $L^{2}$ norm. To obtain good approximation in the $H^{1}$ norm, one needs to add correctors. However, the usual periodic cell problems are not valid, as $a^{\varepsilon}$ is not periodic. One could compute the periodic correctors corresponding to the tensor $a_{p e r}^{\varepsilon}$ and use them to correct the homogenized solution. This will be a good approximation at the large scale but will fail at the fine scale close to the defect. Following the approach in [11], a new corrector can be computed by adding a term to the periodic correctors as follows. Let $\chi^{j} \in W_{\text {per }}^{1}(Y)$ be the classical periodic correctors that satisfy the cell problems

$$
\int_{Y} a_{p e r}^{\varepsilon}(x) \nabla \chi^{j} \cdot \nabla v \mathrm{~d} y=-\int_{Y} a_{p e r}^{\varepsilon}(x) e_{j} \nabla v \mathrm{~d} y \quad \forall v \in W_{p e r}^{1}(Y) .
$$

Then, the additional term will be the solution of a Dirichlet boundary value problem in $K_{n}=[-n \varepsilon, n \varepsilon]^{2}$, where $n$ is large enough so that the effects of the defect are negligible at the boundary of $K_{n}$. The problem reads as follows: find $\chi_{b}^{j} \in H_{0}^{1}\left(K_{n}\right)$, the solution of

$$
\int_{K_{n}} a^{\varepsilon}(x) \nabla \chi_{b}^{j} \cdot \nabla v \mathrm{~d} x=-\int_{K_{n}} b^{\varepsilon}(x)\left(e_{j}+\nabla \chi^{j}\right) \cdot \nabla v \mathrm{~d} x \quad \forall v \in H_{0}^{1}\left(K_{n}\right) .
$$

One can extend $\chi^{j}$ periodically to $K_{n}$ and obtain a corrector $\tilde{\chi}^{j}(x)=\chi^{j}(x)+\chi_{b}^{j}(x)$ for all $x \in K_{n}$. In this numerical example, we compute the FE-HMM solution and add to it either the periodic correctors $\chi$ or the modified correctors $\tilde{\chi}$. We then compare these two solutions with the optimization based solution presented in this paper. We will take the same oscillatory data as given in $\left[11\right.$, sect. 4.]. Let $\Omega=[-1,1]^{2}$, and define

$$
\begin{aligned}
a_{p e r}^{\varepsilon}\left(x_{1}, x_{2}\right) & =3+\cos \left(2 \pi \frac{x_{1}}{\varepsilon}\right)+\sin \left(2 \pi \frac{x_{2}}{\varepsilon}\right), \\
b^{\varepsilon}\left(x_{1}, x_{2}\right) & =10 \exp \left(-\left(\frac{x_{1}^{2}}{\varepsilon^{2}}+\frac{x_{2}^{2}}{\varepsilon^{2}}\right)\right) \\
f\left(x_{1}, x_{2}\right) & =\sin \left(\pi x_{1}\right) \cos \left(\pi x_{2}\right) .
\end{aligned}
$$

We use a uniform triangular mesh and compute a reference solution on a very fine mesh. We compute the periodic correctors on $\mathcal{T}_{h}(Y)$ and extend them to $[-n \varepsilon, n \varepsilon]^{2}$ 
TABLE 1

Relative error in $\omega_{1}$ and $[-\varepsilon, \varepsilon]^{2}$, with $\varepsilon=1 / 5$ and $\varepsilon=1 / 10$, between the reference solution and the periodic solution, the nonperiodic reconstructed solution, and the optimization based solution.

\begin{tabular}{|l|l|c|c|}
\hline & \multicolumn{1}{|c|}{ Method } & Rel. error in $\omega_{1}$ & Rel. error in $[-\varepsilon, \varepsilon]^{2}$ \\
\hline \multirow{3}{*}{$\varepsilon=1 / 5$} & periodic correctors & 0.436 & 1.589 \\
& nonperiodic correctors & 0.396 & 0.992 \\
& optimization based coupling & 0.119 & 0.030 \\
\hline \multirow{3}{*}{$\varepsilon=1 / 10$} & periodic correctors & 0.281 & 1.076 \\
& nonperiodic correctors & 0.260 & 0.720 \\
& optimization based coupling & 0.039 & 0.006 \\
\hline
\end{tabular}

where $n$ is sufficiently large. The terms $\chi_{b}$ are then computed on $[-n \varepsilon, n \varepsilon]^{2}$ with Dirichlet boundary conditions, and adding them to $\chi$, we obtain the nonperiodic correctors $\tilde{\chi}$. In each macroelement $K$ we define a mesh $\mathcal{T}_{h_{\text {rec }}}(K)$, obtained by uniform refinement of $K$ until the mesh size $h_{r e c}$ is smaller than or equal to $h$. The two reconstructed solutions read as

$$
\begin{aligned}
& u_{H}^{\varepsilon, r e c}(x)=u^{H}(x)+\sum_{i=1}^{d} \varepsilon \chi^{i, h}\left(x, \frac{x}{\varepsilon}\right) \frac{\partial u^{H}(x)}{\partial x_{i}}, \\
& \tilde{u}_{H}^{\varepsilon, r e c}(x)=u^{H}(x)+\sum_{i=1}^{d} \varepsilon \tilde{\chi}^{i, h}\left(x, \frac{x}{\varepsilon}\right) \frac{\partial u^{H}(x)}{\partial x_{i}}
\end{aligned}
$$

where both correctors are defined on $[-n \varepsilon, n \varepsilon]^{2}$ with mesh size $h$ and interpolated to $\mathcal{T}_{h_{K}}(K)$. In the coupling method, the fine scale region $\omega_{1}$ will be centered around the defect; as its size is $\varepsilon$, we set $\omega=[-1 / 4,1 / 4]^{2}$ and $\omega_{1}=[-1 / 2,1 / 2]^{2}$. The mesh size in $\omega_{1}$ is equal to $h_{r e c}$, and the mesh size in the coarse region $\Omega \backslash \omega_{1}$ is $H$. We recall that the fine scale reference solution is given by

$$
\bar{u}_{\tilde{h} H}= \begin{cases}u_{1, \tilde{h}} & \text { in } \omega_{+}, \\ u_{2, H}^{r e c} & \text { in } \Omega \backslash \omega_{+},\end{cases}
$$

where we have chosen $\omega_{+}=[-3 / 8,3 / 8]^{2}$. We compute the error between the reference solution and the numerical solutions $u_{H}^{\varepsilon, r e c}, \tilde{u}_{H}^{\varepsilon, \text { rec }}$, and $\bar{u}_{\tilde{h} H}$ in $\omega_{1}$ and in $[-\varepsilon, \varepsilon]^{2}$. We first take $\varepsilon=1 / 5, H=1 / 16$, and a micro-DOF of $N_{\text {micro }}=\frac{1}{32^{2}}$.

We look at the relative error between the reference solution and the reconstructed solution $u_{H}^{\varepsilon, r e c}$ (resp., $\tilde{u}_{H}^{\varepsilon, r e c}$ ) for the periodic correctors (resp., nonperiodic),

$$
\frac{\left\|\nabla\left(u^{\varepsilon}-u_{H}^{\varepsilon, r e c}\right)\right\|_{\mathrm{L}^{2}\left(\omega_{1}\right)}}{\left\|\nabla u^{\varepsilon}\right\|_{\mathrm{L}^{2}\left(\omega_{1}\right)}} .
$$

As expected (see, e.g., [11]), the errors with the two reconstructed solutions are similar in the far field, and one should look at the error around the defects to see the advantage of the correctors $\tilde{\chi}$. In Table 1 , we see the relative errors for the three methods for $\varepsilon=1 / 5$ and $\varepsilon=1 / 10$. In Figure 6 , we display the error in $\omega_{1}$ between the reference solution and the numerical fine scale solutions obtained with the periodic correctors 6(a), nonperiodic correctors 6(b), and the optimization based method 6(c). While the errors between the periodic and nonperiodic methods are similar in $\omega_{1}$, the difference is more important in $[-\varepsilon, \varepsilon]^{2}$, near the defect. There is, however, a 


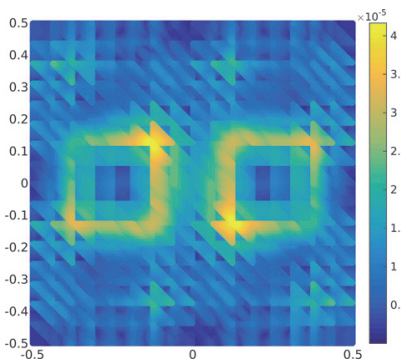

(a)

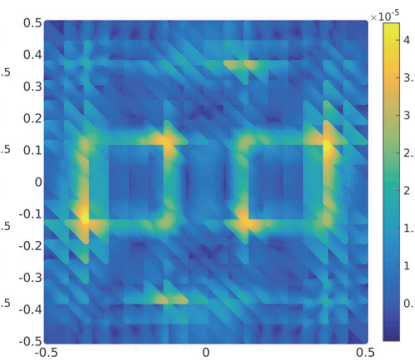

(b)

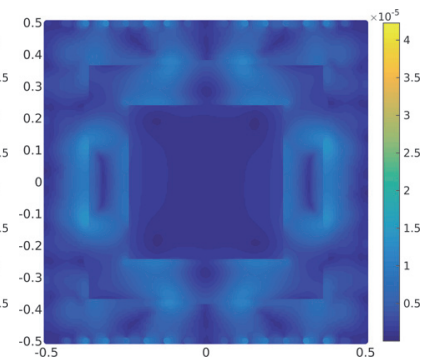

(c)

FIG. 6. Error in $\omega_{1}$ between the reference solution and the numerical fine scale solution obtained with periodic correctors (a), with nonperiodic correctors (b), and by the coupling (c).

significant improvement when the optimization based coupling method is used. This is to be expected, as a fine scale solver is used in $\omega_{1}$ and is coupled with a coarse scale solver. The strength of the method is that it produces a good $H^{1}$ approximation of the fine scale solution on $\Omega$, but allows for a large mesh size $H$ in $\Omega \backslash \omega_{1}$. We note that in [11], the same macro- and micro-DOF were used, with macromesh size of $1 / 1000$, leading to a smaller discretization error and a larger difference between the periodic correctors and the nonperiodic correctors. Setting $H$ to such a small value is not necessary in our experiments, as we only need a fine mesh in $\omega_{1}$ and want to take full advantage of the homogenization techniques in the region with scale separation.

\section{Appendix A. Inequalities.}

A.1. Continuous inequalities. Let us start by recalling the Caccioppoli inequality [25]. Let $\omega \subset \omega_{1}$ be subdomains of $\Omega$ with $\tau=\operatorname{dist}\left(\partial \omega, \partial \omega_{1}\right)$ and set $\Gamma=\partial \Omega$. For a tensor $a$, we define the set of $a$-harmonic functions by $\mathcal{H}\left(\omega_{1}\right)$, which consists of functions $u \in L^{2}\left(\omega_{1}\right) \cap H_{\mathrm{loc}}^{1}\left(\omega_{1}\right)$ such that

$$
B_{1}(u, v)=\int_{\omega_{1}} a \nabla u \cdot \nabla v \mathrm{~d} x=0 \quad \forall v \in \mathcal{C}_{0}^{\infty}\left(\omega_{1}\right)
$$

where $H_{\text {loc }}^{1}$ is defined by

$$
H_{\mathrm{loc}}^{1}\left(\omega_{1}\right):=\left\{u \in H^{1}(O) \mid \text { for any open set } O \text { with } \bar{O} \subset \omega_{1}\right\} .
$$

If the domains have shared boundaries, i.e., $\partial \omega_{1} \cap \Gamma \neq \emptyset$, we construct the space of $a$-harmonic functions by $\mathcal{H}_{0}\left(\omega_{1}\right)$, which consists of functions $u \in \mathcal{H}\left(\omega_{1}\right)$ with zero boundary condition on $\partial \omega_{1} \cap \Gamma$. We recall that $\Gamma_{1}=\partial \omega_{1} \backslash \Gamma$.

Theorem A.1 (Caccioppoli inequality [25]). Let $u \in \mathcal{H}\left(\omega_{1}\right)$; then

$$
\|\nabla u\|_{\mathrm{L}^{2}(\omega)} \leq \frac{\beta^{1 / 2}}{\alpha^{1 / 2} \tau}\|u\|_{\mathrm{L}^{2}\left(\omega_{1}\right)},
$$

where $\alpha$ and $\beta$ are the coercivity constants of the tensor a given by (2.2) and $\tau$ is the width of the domain $\omega_{0}$.

We note that an elliptic problem with a nonnull right-hand side can also be considered, and we refer the reader to [25] for details. Next we generalize the above result in order to have only the overlapping domain in the right-hand side. 
Lemma A.2. Let $\omega_{0}=\omega_{1} \backslash \bar{\omega}$. Let $u \in \mathcal{H}\left(\omega_{1}\right)$; then

$$
\|\nabla u\|_{\mathrm{L}^{2}(\omega)} \leq \frac{\beta^{1 / 2}}{\alpha^{1 / 2} \tau}\|u\|_{\mathrm{L}^{2}\left(\omega_{0}\right)},
$$

where $\alpha$ and $\beta$ are given by (2.2) and $\tau$ is the width of the domain $\omega_{0}$.

Proof. Let $\eta \in \mathcal{C}_{0}^{1}\left(\omega_{1}\right)$ be a cutoff function with $\eta=1$ in $\bar{\omega}, \eta=0$ in $\partial \omega_{1}$, and $|\nabla \eta| \leq 1 / \tau$. Further, $\eta=0$ on $\Gamma_{1}$, and $\operatorname{supp}(\nabla \eta) \subset \omega_{0}$. Then, it holds that $\eta^{2} u \in H_{0}^{1}\left(\omega_{1}\right)$ and

$$
\int_{\omega_{1}} a \nabla u \cdot \nabla\left(\eta^{2} u\right) \mathrm{d} x=0
$$

Then,

$$
0=\int_{\omega_{1}} a \nabla u \cdot \nabla\left(\eta^{2} u\right) \mathrm{d} x=2 \int_{\omega_{1}} a \nabla u \cdot \nabla \eta \eta u \mathrm{~d} x+\int_{\omega_{1}} a \nabla u \cdot \nabla u \eta^{2} \mathrm{~d} x .
$$

Using the ellipticity of $a$ and the definition of $\eta$, it holds that

$$
\alpha\|\nabla u\|_{\mathrm{L}^{2}(\omega)}^{2} \leq \int_{\omega_{1}} a \nabla(\eta u) \cdot \nabla(\eta u) \mathrm{d} x
$$

and

$$
\begin{aligned}
\int_{\omega_{1}} a \nabla(\eta u) \cdot \nabla(\eta u) \mathrm{d} x & =\int_{\omega_{1}} a \nabla(\eta u) \cdot \nabla(\eta u) \mathrm{d} x-\int_{\omega_{1}} a \nabla u \cdot \nabla\left(\eta^{2} u\right) \mathrm{d} x \\
& =\int_{\omega_{1}} a \nabla(\eta u) \cdot \nabla(\eta u) \mathrm{d} x-2 \int_{\omega_{1}} a \nabla u \cdot \nabla \eta \eta u \mathrm{~d} x-\int_{\omega_{1}} a \nabla u \cdot \nabla u \eta^{2} \mathrm{~d} x \\
& =\int_{\omega_{1}} a \nabla \eta \cdot \nabla \eta u^{2} \mathrm{~d} x \\
& =\int_{\omega_{0}} a \nabla \eta \cdot \nabla \eta u^{2} \mathrm{~d} x \\
& \leq \frac{\beta}{\tau^{2}} \int_{\omega_{0}} u^{2} \mathrm{~d} x=\frac{\beta}{\tau^{2}}\|u\|_{\mathrm{L}^{2}\left(\omega_{0}\right)}^{2} .
\end{aligned}
$$

In the next lemma, we prove a strong version of the Cauchy-Schwarz inequality. We recall the problems for the state variables: find $v_{i} \in H_{D}^{1}\left(\omega_{i}\right)$, the solution of

$$
\begin{array}{rlrl}
-\operatorname{div}\left(a_{i} \nabla v_{i}\right) & =0 & & \text { in } \omega_{i}, \\
v_{i}=\theta_{i} & & \text { on } \Gamma_{i}, \\
v_{i}=0 & & \text { on } \partial \omega_{i} \cap \Gamma_{D}, \\
n_{i} \cdot\left(a_{i} \nabla v_{i}\right) & =0 & & \text { on } \partial \omega_{i} \cap \Gamma_{N},
\end{array}
$$

where $a_{1}=a_{1}^{\varepsilon}$ and $a_{2}=a_{2}^{0}$.

Lemma A.3 (strong Cauchy-Schwarz). Let $v_{1}^{\varepsilon} \in H_{D}^{1}\left(\omega_{1}\right)$ and $v_{2}^{0} \in H_{D}^{1}\left(\omega_{2}\right)$ be solutions of (A.1) for $i=1,2$. Then, there exist an $\varepsilon_{0}>0$ and a positive constant $C_{s}<1$ such that for all $\varepsilon \leq \varepsilon_{0}$, it holds that

$$
\int_{\omega_{0}} v_{1}^{\varepsilon} v_{2}^{0} \mathrm{~d} x \leq C_{s}\left\|v_{1}^{\varepsilon}\right\|_{\mathrm{L}^{2}\left(\omega_{0}\right)}\left\|v_{2}^{0}\right\|_{\mathrm{L}^{2}\left(\omega_{0}\right)}
$$

Copyright $@$ by SIAM. Unauthorized reproduction of this article is prohibited. 
Proof. We reason by contradiction. Suppose there exists a sequence of $\left\{\varepsilon_{n}\right\}_{n \geq 1}$ that tends to zero such that

$$
\int_{\omega_{0}} v_{1}^{\varepsilon_{n}} v_{2}^{0} \mathrm{~d} x>C_{n}\left\|v_{1}^{\varepsilon_{n}}\right\|_{\mathrm{L}^{2}\left(\omega_{0}\right)}\left\|v_{2}^{0}\right\|_{\mathrm{L}^{2}\left(\omega_{0}\right)} \quad \forall n \geq 1
$$

for any sequence $\left\{C_{n}\right\}_{n \geq 1}$ that tends to 1 , with $C_{n}<1$. Without loss of generality, we can normalize the vectors $v_{1}^{\varepsilon_{n}}$ and $v_{2}$ and obtain

$$
\left\|v_{1}^{\varepsilon_{n}}\right\|_{\mathrm{L}^{2}\left(\omega_{0}\right)}=1, \quad\left\|v_{2}^{0}\right\|_{\mathrm{L}^{2}\left(\omega_{0}\right)}=1, \quad \text { and } \quad\left(v_{1}^{\varepsilon_{n}}, v_{2}^{0}\right)_{\mathrm{L}^{2}\left(\omega_{0}\right)}:=\int_{\omega_{0}} v_{1}^{\varepsilon_{n}} v_{2}^{0} \mathrm{~d} x \rightarrow 1 .
$$

As the sequence of tensors $\left\{a_{1}^{\varepsilon_{n}}\right\}_{n \geq 1} \in\left(L^{\infty}\left(\omega_{1}\right)\right)^{d \times d}$ is bounded and uniformly elliptic, by the $H$-convergence, there exist a subsequence of $\left\{\varepsilon_{n}\right\}_{n \geq 1}$ still denoted by $\left\{\varepsilon_{n}\right\}_{n \geq 1}$ and a tensor $a_{1}^{0} \in\left(L^{\infty}\left(\omega_{1}\right)\right)^{d \times d}$ bounded and uniformly elliptic such that $\left\{a_{1}^{\varepsilon_{n}}\right\}_{n \geq 1}$ $H$-converges to $a_{1}^{0}$. By definition of the $H$-convergence, the solution $v_{1}^{\varepsilon_{n}}$ of (A.1)-for the subsequence $\left\{\varepsilon_{n}\right\}$-is such that

(i) $v_{1}^{\varepsilon_{n}} \rightarrow v_{1}^{0}$ in $H^{1}\left(\omega_{1}\right)$ and

(ii) $a_{1}^{\varepsilon_{n}} \nabla v_{1}^{\varepsilon_{n}} \rightarrow a_{1}^{0} \nabla v_{1}^{0}$ in $L^{2}\left(\omega_{1}\right)^{d}$, where $v_{1}^{0}$ is the unique solution of

$$
\begin{array}{rlrl}
-\operatorname{div}\left(a_{1}^{0} \nabla v_{1}^{0}\right) & =0 & & \text { in } \omega_{1}, \\
v_{1}^{0}=\theta_{1} & & \text { on } \Gamma_{1}, \\
v_{1}^{0} & =0 & & \text { on } \partial \omega_{1} \cap \Gamma_{D}, \\
n_{1} \cdot\left(a_{1}^{0} \nabla v_{1}^{0}\right) & =0 & & \text { on } \partial \omega_{1} \cap \Gamma_{N} .
\end{array}
$$

As $H^{1}\left(\omega_{1}\right)$ is compactly embedded in $L^{2}\left(\omega_{1}\right)$, strong convergence in $L^{2}$ of $v_{1}^{\varepsilon_{n}}$ to $v_{1}^{0}$, for a subsequence of $\left\{\varepsilon_{n}\right\}_{n \geq 1}$, is achieved, i.e.,

$$
v_{1}^{\varepsilon_{n}} \rightarrow v_{1}^{0} \text { in } L^{2}\left(\omega_{1}\right) .
$$

By the continuity of the norm, we have that

$$
\lim _{n \rightarrow \infty}\left(v_{1}^{\varepsilon_{n}}, v_{2}\right)_{\mathrm{L}^{2}\left(\omega_{0}\right)}=\left(v_{1}^{0}, v_{2}\right)_{\mathrm{L}^{2}\left(\omega_{0}\right)}, \quad\left\|v_{1}^{0}\right\|_{\mathrm{L}^{2}\left(\omega_{0}\right)} \leq 1, \quad \text { and } \quad\left(v_{1}^{0}, v_{2}\right)_{\mathrm{L}^{2}\left(\omega_{0}\right)}=1 .
$$

As

$$
1=\left(v_{1}^{0}, v_{2}\right)_{\mathrm{L}^{2}\left(\omega_{0}\right)} \leq\left\|v_{1}^{0}\right\|_{\mathrm{L}^{2}\left(\omega_{0}\right)}\left\|v_{2}\right\|_{\mathrm{L}^{2}\left(\omega_{0}\right)} \leq 1,
$$

we must have that $\left\|v_{1}^{0}\right\|_{\mathrm{L}^{2}\left(\omega_{0}\right)}\left\|v_{2}\right\|_{\mathrm{L}^{2}\left(\omega_{0}\right)}=1$ and hence $\left\|v_{1}^{0}\right\|_{\mathrm{L}^{2}\left(\omega_{0}\right)}=1$. The previous inequalities become equalities, i.e.,

$$
1=\left(v_{1}^{0}, v_{2}\right)_{\mathrm{L}^{2}\left(\omega_{0}\right)}=\left\|v_{1}^{0}\right\|_{\mathrm{L}^{2}\left(\omega_{0}\right)}\left\|v_{2}\right\|_{\mathrm{L}^{2}\left(\omega_{0}\right)} .
$$

An equality in Cauchy-Schwarz is possible if and only if $v_{1}^{0}$ and $v_{2}$ are linearly dependent, that is, there exists a constant $c>0$ such that $v_{1}^{0}=c v_{2}$ a.e. in $\omega_{0}$. As the norms of $v_{1}^{0}$ and $v_{2}$ are equal to 1 , we can easily conclude that $c= \pm 1$ and that $v_{1}^{0}$ $= \pm v_{2}$ a.e. in $\omega_{0}$. Finally, as $\left(v_{1}^{0}, v_{2}\right)_{\mathrm{L}^{2}\left(\omega_{0}\right)}=1$, it holds that $v_{1}^{0}=v_{2}$.

Both $v_{1}^{0}$ and $v_{2}$ are solutions of a homogenized equation and are equal on the overlap, so we can combine them into a homogenized solution on the entire domain $\Omega$. Further, the tensors $a_{2}^{0}$ and $a_{1}^{0}$ are equal in $\omega_{0}$. Indeed, let us continuously extend the tensors $a_{2}^{\varepsilon}$ and $a_{1}^{\varepsilon}$ to the domain $\Omega$. The tensor $a_{1}^{\varepsilon} H$-converges to the tensor $a_{1}^{0}$, and the tensor $a_{2}^{\varepsilon} H$-converges to $a_{2}^{0}$ in $\Omega$. It holds that $a_{2}^{\varepsilon}=a_{1}^{\varepsilon}$ in $\omega_{0}$, and using the 
locality of $H$-convergence $[34,15]$, we can conclude that $a_{2}^{0}=a_{1}^{0}$ in $\omega_{2}$. Thus they are equal in the overlap.

Let us split $\omega_{0}$ into two disjoint sets $\omega_{0}^{1}$ and $\omega_{0}^{2}$ such that $\omega \subset \subset \omega \cup \omega_{0}^{1} \subset \subset \omega \cup \omega_{0}$. As the solutions $v_{1}^{0}$ and $v_{2}$ are equal in $\omega_{0}$, we can construct a smooth function $\bar{v}$ over $\Omega$ as

$$
\bar{v}(x)= \begin{cases}v_{1}^{0}(x) & \text { if } x \in \omega \cup \omega_{0}^{1}, \\ v_{2}(x) & \text { if } x \in \omega_{2} \backslash \omega_{0}^{1} .\end{cases}
$$

The function $\bar{v}$ is in $H_{D}^{1}(\Omega)$, has zero Neumann boundary condition on $\Gamma_{N}$, and satisfies

$$
\int_{\Omega} \bar{a}^{0} \nabla \bar{v} \cdot \nabla w \mathrm{~d} x=0 \quad \forall w \in H_{D}^{1}(\Omega),
$$

where the tensor $\bar{a}^{0}$ is given by

$$
\bar{a}^{0}= \begin{cases}a_{1}^{0} & \text { in } \omega \cup \omega_{0}^{1}, \\ a_{2}^{0} & \text { in } \omega_{2} \backslash \omega_{0}^{1} .\end{cases}
$$

The solution $\bar{v}$ must be zero everywhere in $\Omega$, i.e., $\bar{v} \equiv 0$, which is a contradiction with $\|\bar{v}\|_{\mathrm{L}^{2}\left(\omega_{0}\right)}=1$.

A.2. Discrete inequalities. Let $\omega \subset \omega_{1} \subset \Omega$, with $\tau=\operatorname{dist}\left(\partial \omega_{1}, \partial \omega\right)$, and consider a partition $\mathcal{T}_{h}$ of $\Omega$ in simplicial or quadrilateral elements $K$, with diameter $h_{K}$ and where the mesh size $h$ is given by $h=\max _{K \in \mathcal{T}_{h}} h_{K}$. Further, we assume that $h$ is smaller than $\tau$ and that $\mathcal{T}_{h}$ is admissible (T1) and shape regular (T2). The inequalities are given for general FE spaces of degree $p \geq 1$.

We give a discrete Caccioppoli inequality for functions $v^{h} \in V^{p}\left(\omega_{1}, \mathcal{T}_{h}\right)$ that are solutions of

$$
B_{1}\left(v^{h}, w^{h}\right):=\int_{\omega_{1}} a \nabla v^{h} \cdot \nabla w^{h} \mathrm{~d} x=0 \quad \forall w^{h} \in V_{0}^{p}\left(\omega_{1}, \mathcal{T}_{h}\right) .
$$

Let us denote by $I_{h}$ the Lagrange interpolant and state a superapproximation useful in the proof of the discrete Caccioppoli inequality.

Lemma A.4. Let $\eta \in \mathcal{C}^{1}\left(\omega_{1}\right)$ with $|\nabla \eta| \leq C \tau^{-1}$. Then for each $v^{h} \in V^{p}\left(\omega_{1}, \mathcal{T}_{h}\right)$ and $K \in \mathcal{T}_{h}$, with $h_{K} \leq \tau$, it holds that

$$
\left\|\eta^{2} v^{h}-I_{h}\left(\eta^{2} v^{h}\right)\right\|_{\mathrm{H}^{1}\left(\omega_{1}\right)} \leq C\left(\frac{h_{K}}{\tau}\left\|\nabla\left(\eta v^{h}\right)\right\|_{\mathrm{L}^{2}(K)}+\frac{h_{K}}{\tau^{2}}\left\|v^{h}\right\|_{\mathrm{L}^{2}(K)}\right) .
$$

Proof. For the proof, see [17, Theorem 2.1].

We recall that local inverse inequalities are valid for functions $v^{h} \in V^{p}\left(\omega_{1}, \mathcal{T}_{h}\right)$; that is,

$$
\left\|\nabla v^{h}\right\|_{\mathrm{L}^{2}(K)} \leq C h_{K}^{-1}\left\|v^{h}\right\|_{\mathrm{L}^{2}(K)},
$$

where the constant $C$ is independent of $h_{K}$.

Lemma A.5 (discrete Caccioppoli inequality for interior domains [35]). Let $v^{h} \in$ $V^{p}\left(\omega_{1}, \mathcal{T}_{h}\right)$ satisfy (A.2) for all $w^{h} \in V_{0}^{p}\left(\omega_{1}, \mathcal{T}_{h}\right)$; it holds that

$$
\left\|\nabla v^{h}\right\|_{\mathrm{L}^{2}(\omega)} \leq C \frac{1}{\tau}\left\|v^{h}\right\|_{\mathrm{L}^{2}\left(\omega_{1}\right)},
$$

where the constant $C$ is independent of $h$.

Copyright $@$ by SIAM. Unauthorized reproduction of this article is prohibited. 
Proof. Let $\eta \in \mathcal{C}_{0}^{1}\left(\omega_{1}\right)$ be a cutoff function with $|\nabla \eta| \leq C \tau^{-1}$. We have that $\eta$ satisfies $\eta \equiv 0$ in $\Omega \backslash \omega_{1}, \eta \equiv 1$ in $\omega$, and $|\nabla \eta| \leq 1 / \tau$ for points in $\omega_{0}$. By the uniform ellipticity of the tensor $a$, it holds that

$$
\alpha\left\|\nabla v^{h}\right\|_{\mathrm{L}^{2}(\omega)}^{2} \leq \int_{\omega_{1}} a \nabla v^{h} \cdot \nabla v^{h} \eta^{2} \mathrm{~d} x .
$$

Using $\eta^{2} v^{h}$ as a test function in (A.2) and expanding the integral, we obtain

$$
\int_{\omega_{1}} a \nabla v^{h} \cdot \nabla\left(\eta^{2} v^{h}\right) \mathrm{d} x=\int_{\omega_{1}} a \nabla v^{h} \cdot \nabla v^{h} \eta^{2} \mathrm{~d} x+2 \int_{\omega_{1}} a \eta \nabla v^{h} \cdot \nabla \eta v^{h} \mathrm{~d} x,
$$

and thus

$$
\begin{aligned}
\int_{\omega_{1}} a \nabla v^{h} \cdot \nabla v^{h} \eta^{2} \mathrm{~d} x & =\int_{\omega_{1}} a \nabla v^{h} \cdot \nabla\left(\eta^{2} v^{h}\right) \mathrm{d} x-2 \int_{\omega_{1}}\left(\eta a^{1 / 2} \nabla v^{h}\right) \cdot\left(v^{h} a^{1 / 2} \nabla \eta\right) \mathrm{d} x \\
& \leq \int_{\omega_{1}} a \nabla v^{h} \cdot \nabla\left(\eta^{2} v^{h}\right) \mathrm{d} x+2 \int_{\omega_{1}}\left(\eta a^{1 / 2} \nabla v^{h}\right) \cdot\left(v^{h} a^{1 / 2} \nabla \eta\right) \mathrm{d} x \\
& \leq B_{1}\left(v^{h}, \eta^{2} v^{h}\right)+\zeta \int_{\omega_{1}} a \nabla v^{h} \cdot \nabla v^{h} \eta^{2} \mathrm{~d} x+\frac{1}{\zeta} \int_{\omega_{1}} a v^{h} \nabla \eta \cdot \nabla \eta v^{h} \mathrm{~d} x \\
& \leq B_{1}\left(v^{h}, \eta^{2} v^{h}\right)+\zeta \int_{\omega_{1}} a \nabla v^{h} \cdot \nabla v^{h} \eta^{2} \mathrm{~d} x+\frac{\beta}{\zeta \tau^{2}}\left\|v^{h}\right\|_{\mathrm{L}^{2}\left(\omega_{1}\right)}^{2} .
\end{aligned}
$$

The last step is to bound the quantity $B_{1}\left(v^{h}, \eta^{2} v^{h}\right)$. Let us consider $I_{h}\left(\eta^{2} v^{h}\right) \in$ $V^{p}\left(\omega_{1}, \mathcal{T}_{h}\right) ;$ it holds that

$$
B_{1}\left(v^{h}, I\left(\eta^{2} v^{h}\right)\right)=0,
$$

and then

$$
\begin{aligned}
B_{1}\left(v^{h}, \eta^{2} v^{h}\right) & =B_{1}\left(v^{h}, \eta^{2} v^{h}-I\left(\eta^{2} v^{h}\right)\right)=\int_{\omega_{1}} a \nabla v^{h} \nabla\left(\eta^{2} v^{h}-I\left(\eta^{2} v^{h}\right)\right) \mathrm{d} x \\
& \leq \beta\left\|\nabla v^{h}\right\|_{\mathrm{L}^{2}\left(\omega_{1}\right)}\left\|\nabla\left(\eta^{2} v^{h}-I\left(\eta^{2} v^{h}\right)\right)\right\|_{\mathrm{L}^{2}\left(\omega_{1}\right)} \\
& \leq \beta \sum_{K \in \mathcal{T}_{h}}\left\|\nabla v^{h}\right\|_{\mathrm{L}^{2}(K)}\left\|\nabla\left(\eta^{2} v^{h}-I\left(\eta^{2} v^{h}\right)\right)\right\|_{\mathrm{L}^{2}(K)} .
\end{aligned}
$$

Using the local inverse inequality (A.3) and Lemma A.4, we obtain

$$
\begin{aligned}
B_{1}\left(v^{h}, \eta^{2} v^{h}\right) & \leq C \beta \sum_{K \in \mathcal{T}_{h}} \frac{1}{h_{K}}\left\|v^{h}\right\|_{\mathrm{L}^{2}(K)}\left(\frac{h_{K}}{\tau}\left\|\nabla\left(\eta v^{h}\right)\right\|_{\mathrm{L}^{2}(K)}+\frac{h_{K}}{\tau^{2}}\left\|v^{h}\right\|_{\mathrm{L}^{2}(K)}\right) \\
& =\beta \sum_{K \in \mathcal{T}_{h}}\left\|v^{h}\right\|_{\mathrm{L}^{2}(K)} \frac{C}{\tau}\left\|\nabla\left(\eta v^{h}\right)\right\|_{\mathrm{L}^{2}(K)}+\frac{C}{\tau^{2}}\left\|v^{h}\right\|_{\mathrm{L}^{2}(K)}^{2} \\
& \leq \beta \sum_{K \in \mathcal{T}_{h}} \frac{C}{\tau^{2}}\left(\frac{1}{\zeta}+1\right)\left\|v^{h}\right\|_{\mathrm{L}^{2}(K)}^{2}+\zeta\left\|\nabla\left(\eta v^{h}\right)\right\|_{\mathrm{L}^{2}(K)}^{2} \\
& \leq \frac{C \beta}{\tau^{2}}\left(\frac{1}{\zeta}+1\right)\left\|v^{h}\right\|_{\mathrm{L}^{2}\left(\omega_{1}\right)}^{2}+\beta \zeta\left\|\eta \nabla v^{h}\right\|_{\mathrm{L}^{2}\left(\omega_{1}\right)}^{2}+\beta \zeta\left\|v^{h} \nabla \eta\right\|_{\mathrm{L}^{2}\left(\omega_{1}\right)}^{2} \\
& \leq \beta\left(\frac{C}{\tau^{2}}\left(\frac{1}{\zeta}+1+\zeta\right)\left\|v^{h}\right\|_{\mathrm{L}^{2}\left(\omega_{1}\right)}^{2}+\zeta\left\|\eta \nabla v^{h}\right\|_{\mathrm{L}^{2}\left(\omega_{1}\right)}^{2}\right) .
\end{aligned}
$$

Copyright $\odot$ by SIAM. Unauthorized reproduction of this article is prohibited. 
Recalling that

$$
\left\|\eta \nabla v^{h}\right\|_{\mathrm{L}^{2}\left(\omega_{1}\right)}^{2}=\int_{\omega_{1}} \nabla v^{h} \cdot \nabla v^{h} \eta^{2} \mathrm{~d} x \leq \frac{1}{\alpha} \int_{\omega_{1}} a \nabla v^{h} \cdot \nabla v^{h} \eta^{2} \mathrm{~d} x
$$

and collecting the previous bounds, it holds that

$$
\begin{aligned}
\int_{\omega_{1}} a \nabla v^{h} \cdot \nabla v^{h} \eta^{2} \mathrm{~d} x \leq & C \frac{\beta}{\tau^{2}}\left(\frac{2}{\zeta}+1+\zeta\right)\left\|v^{h}\right\|_{\mathrm{L}^{2}\left(\omega_{1}\right)}^{2} \\
& +\zeta\left(\frac{\beta}{\alpha}+1\right) \int_{\omega_{1}} a \nabla v^{h} \cdot \nabla v^{h} \eta^{2} \mathrm{~d} x .
\end{aligned}
$$

This gives, for $\zeta \neq 1 /(\beta / \alpha+1)$,

$$
\left(1-\zeta\left(\frac{\beta}{\alpha+1}\right)\right) \int_{\omega_{1}} a \nabla v^{h} \cdot \nabla v^{h} \eta^{2} \mathrm{~d} x \leq C \frac{\beta}{\tau^{2}}\left(\frac{2}{\zeta}+1+\zeta\right)\left\|v^{h}\right\|_{\mathrm{L}^{2}\left(\omega_{1}\right)}^{2},
$$

and finally

$$
\left\|\nabla v^{h}\right\|_{\mathrm{L}^{2}(\omega)}^{2} \leq \frac{C}{(1-\zeta(\beta / \alpha+1))}\left(\frac{2}{\zeta}+1+\zeta\right) \frac{\beta}{\alpha \tau^{2}}\left\|v^{h}\right\|_{\mathrm{L}^{2}\left(\omega_{1}\right)}^{2} .
$$

Assume now that $\partial \omega \cap \Gamma \neq \emptyset$. A discrete Caccioppoli inequality can be proved.

LEmma A.6 (discrete Caccioppoli inequality for domains with shared boundaries). Let $v^{h} \in V^{p}\left(\omega_{1}, \mathcal{T}_{h}\right)$ satisfy (A.2) for all $w^{h} \in V_{0}^{p}\left(\omega_{1}, \mathcal{T}_{h}\right)$. Further assume that $v^{h}=0$ on $\partial \omega_{1} \cap \Gamma$. Then it holds that

$$
\left\|\nabla v^{h}\right\|_{L^{2}(\omega)} \leq C \frac{1}{\tau}\left\|v^{h}\right\|_{L^{2}\left(\omega_{1}\right)},
$$

where the constant $C$ is independent of $h$.

Proof. Now we consider a cutoff function $\eta$ such that $\eta \equiv 1$ in $\bar{\omega}, \eta \equiv 0$ in $\Omega \backslash \omega_{1}$, and $\eta \equiv 0$ on $\partial \omega_{1} \cap \Omega$. We can then follow the proof of Lemma A.5, as

$$
B_{1}\left(v^{h}, \eta^{2} v^{h}\right)=\int_{\omega_{1}} a \nabla v^{h} \cdot \nabla\left(\eta^{2} v^{h}\right) \mathrm{d} x=0 .
$$

We now show that the strong Cauchy-Schwarz inequality lemma, Lemma A.3, is still valid for discrete functions. For simplicity in the notation, we omit the $\varepsilon$ dependency in $v_{1}$.

Lemma A.7. Let $\varepsilon<\varepsilon_{0}$ and $C_{s}<1$ be given by the strong Cauchy-Schwarz lemma, Lemma A.3, and let $v_{1, \tilde{h}} \in V_{D}^{p}\left(\omega_{1}, \mathcal{T}_{\tilde{h}}\right)$ and $v_{2, H} \in V_{D}^{p}\left(\omega_{2}, \mathcal{T}_{H}\right)$ be numerical solutions of (4.11). There exist $\tilde{h}_{0}>0$ and $H_{0}>0$ such that

$$
\int_{\omega_{0}} v_{1, \tilde{h}} v_{2, H} \mathrm{~d} x \leq C_{s}\left\|v_{1, \tilde{h}}\right\|_{\mathrm{L}^{2}\left(\omega_{0}\right)}\left\|v_{2, H}\right\|_{\mathrm{L}^{2}\left(\omega_{0}\right)} \quad \forall \tilde{h}<\tilde{h}_{0}, H<H_{0} .
$$

Proof. Let $\left\{\tilde{h}_{n}, H_{n}\right\}_{n \geq 1}$ be a sequence of mesh sizes converging to zero. We have strong convergence in $L^{2}$, for a subsequence of $\left\{\tilde{h}_{n}, H_{n}\right\}_{n \geq 1}$ still denoted by $\left\{\tilde{h}_{n}, H_{n}\right\}_{n \geq 1}$, of the numerical solutions $v_{1, \tilde{h}_{n}}$ and $v_{2, H_{n}}$ to the exact solutions $v_{1}$ and $v_{2}$, respectively. Thus

$$
\lim _{n \rightarrow \infty} \int_{\omega_{0}} v_{1, \tilde{h}_{n}} v_{2, H_{n}} \mathrm{~d} x=\int_{\omega_{0}} v_{1} v_{2} \mathrm{~d} x
$$

Copyright (c) by SIAM. Unauthorized reproduction of this article is prohibited. 
and

$$
\begin{aligned}
& \lim _{n \rightarrow \infty}\left\|v_{1, \tilde{h}_{n}}\right\|_{\mathrm{L}^{2}\left(\omega_{0}\right)}=\left\|v_{1}\right\|_{\mathrm{L}^{2}\left(\omega_{0}\right)}, \\
& \lim _{n \rightarrow \infty}\left\|v_{2, H_{n}}\right\|_{\mathrm{L}^{2}\left(\omega_{0}\right)}=\left\|v_{2}\right\|_{\mathrm{L}^{2}\left(\omega_{0}\right)} .
\end{aligned}
$$

We recall that the strong Cauchy-Schwarz inequality is valid for $v_{1}$ and $v_{2}$; there exist an $\varepsilon_{0}$ and a constant $C_{s}<1$ such that for all $\varepsilon \leq \varepsilon_{0}$, it holds that

$$
\int_{\omega_{0}} v_{1} v_{2} \mathrm{~d} x \leq C_{s}\left\|v_{1}\right\|_{\mathrm{L}^{2}\left(\omega_{0}\right)}\left\|v_{2}\right\|_{\mathrm{L}^{2}\left(\omega_{0}\right)} .
$$

Then, using the strong Cauchy-Schwarz inequality for $v_{1}$ and $v_{2}$, it holds that

$$
\begin{aligned}
\lim _{n \rightarrow \infty} \int_{\omega_{0}} v_{1, \tilde{h}_{n}} v_{2, H_{n}} \mathrm{~d} x & =\int_{\omega_{0}} v_{1} v_{2} \mathrm{~d} x \\
& \leq C_{s}\left\|v_{1}\right\|_{\mathrm{L}^{2}\left(\omega_{0}\right)}\left\|v_{2}\right\|_{\mathrm{L}^{2}\left(\omega_{0}\right)} \\
& =\lim _{n \rightarrow \infty} C_{s}\left\|v_{1, \tilde{h}_{n}}\right\|_{\mathrm{L}^{2}\left(\omega_{0}\right)}\left\|v_{2, H_{n}}\right\|_{\mathrm{L}^{2}\left(\omega_{0}\right)} .
\end{aligned}
$$

Then, there exist an $\varepsilon_{0}>0$ and a constant $C_{s}<1$ such that for all $\varepsilon \leq \varepsilon_{0}$, there exist $\tilde{h}_{0}>0$ and $H_{0}>0$ such that

$$
\int_{\omega_{0}} v_{1, \tilde{h}} v_{2, H} \mathrm{~d} x \leq C_{s}\left\|v_{1, \tilde{h}}\right\|_{\mathrm{L}^{2}\left(\omega_{0}\right)}\left\|v_{2, H}\right\|_{\mathrm{L}^{2}\left(\omega_{0}\right)} \quad \forall \tilde{h} \leq \tilde{h}_{0}, H \leq H_{0} .
$$

\section{REFERENCES}

[1] A. AbDulLE, On a priori error analysis of fully discrete heterogeneous multiscale FEM, Multiscale Model. Simul., 4 (2005), pp. 447-459, https://doi.org/10.1137/040607137.

[2] A. AbDulle, The finite element heterogeneous multiscale method: A computational strategy for multiscale PDEs, in Multiple Scales Problems in Biomathematics, Mechanics, Physics, and Numerics, GAKUTO Internat. Ser. Math. Sci. Appl. 31, Gakkōtosho, Tokyo, 2009, pp. $133-181$.

[3] A. AbDulle, A priori and a posteriori error analysis for numerical homogenization: A unified framework, in Multiscale Problems, Ser. Contemp. Appl. Math. CAM 16, Higher Education Press, Beijing, 2011, pp. 280-305.

[4] A. Abdulle, Discontinuous Galerkin finite element heterogeneous multiscale method for elliptic problems with multiple scales, Math. Comp., 81 (2012), pp. 687-713.

[5] A. AbDulle And O. JeCKeR, An optimization-based, heterogeneous to homogeneous coupling method, Commun. Math. Sci., 13 (2015), pp. 1639-1648.

[6] A. AbDulle And O. JeCKer, On heterogeneous coupling of multiscale methods for problems with and without scale separation, submitted.

[7] A. Abdulle And A. Nonnenmacher, Adaptive finite element heterogeneous multiscale method for homogenization problems, Comput. Methods Appl. Mech. Engrg., 200 (2011), no. 3740, pp. $2710-2726$.

[8] D. N. Arnold, F. Brezzi, B. Cockburn, and L. D. Marini, Unified analysis of discontinuous Galerkin methods for elliptic problems, SIAM J. Numer. Anal., 39 (2002), pp. 1749-1779, https://doi.org/10.1137/S0036142901384162.

[9] I. BABUŠKa ANd R. Lipton, $L^{2}$-global to local projection: An approach to multiscale analysis, Math. Models Methods Appl. Sci., 21 (2011), pp. 2211-2226.

[10] A. Bensoussan, J.-L. Lions, and G. Papanicolaou, Asymptotic Analysis for Periodic Structures, North-Holland, Amsterdam, 1978.

[11] X. Blanc, C. Le Bris, and P.-L. Lions, A possible homogenization approach for the numerical simulation of periodic microstructures with defects, Milan J. Math., 80 (2012), pp. 351-367.

[12] F. BREzZI, On the existence, uniqueness and approximation of saddle-point problems arising from Lagrangian multipliers, Rev. Française Automat. Informat. Recherche Opérationelle Sér. Rouge, 8 (1974), pp. 129-151.

Copyright $@$ by SIAM. Unauthorized reproduction of this article is prohibited. 
[13] Z. Chen And H. Chen, Pointwise error estimates of discontinuous Galerkin methods with penalty for second-order elliptic problems, SIAM J. Numer. Anal., 42 (2004), pp. 11461166, https://doi.org/10.1137/S0036142903421527.

[14] P. G. Ciarlet, The Finite Element Method for Elliptic Problems, Stud. Math. Appl. 4, NorthHolland, Amsterdam, New York, 1978.

[15] D. Cioranescu And P. Donato, An Introduction to Homogenization, Oxford Lecture Ser. Math. Appl. 17, The Clarendon Press, Oxford University Press, New York, 1999.

[16] M. D'Elia, M. Perego, P. Bochev, and D. Littlewood, A coupling strategy for nonlocal and local diffusion models with mixed volume constraints and boundary conditions, Comput. Math. Appl., 71 (2016), pp. 2218-2230.

[17] A. Demlow, J. Guzmán, And A. H. Schatz, Local energy estimates for the finite element method on sharply varying grids, Math. Comp., 80 (2011), pp. 1-9, https://doi.org/10. 1090/S0025-5718-2010-02353-1.

[18] D. A. Di Pietro And A. ERn, Mathematical Aspects of Discontinuous Galerkin Methods, Springer, Heidelberg, 2012.

[19] M. Discacciati, P. Gervasio, and A. Quarteroni, The interface control domain decomposition (ICDD) method for elliptic problems, SIAM J. Control Optim., 51 (2013), pp. 3434-3458, https://doi.org/10.1137/120890764.

[20] W. E And B. EngQuist, The heterogeneous multiscale methods, Commun. Math. Sci., 1 (2003), pp. $87-132$.

[21] W. E, P. Ming, And P. Zhang, Analysis of the heterogeneous multiscale method for elliptic homogenization problems, J. Amer. Math. Soc., 18 (2005), pp. 121-156.

[22] Y. Efendiev and T. Y. Hou, Multiscale Finite Element Methods. Theory and Applications, Surv. Tutor. Appl. Math. Sci. 4, Springer, New York, 2009.

[23] M. G. D. Geers, V. G. Kouznetsova, and W. A. M. Brekelmans, Multi-scale computational homogenization: Trends and challenges, J. Comput. Appl. Math., 234 (2010), pp. 21752182.

[24] P. Gervasio, J.-L. Lions, and A. Quarteroni, Heterogeneous coupling by virtual control methods, Numer. Math., 90 (2000), pp. 241-264.

[25] M. Giaquinta, Multiple Integrals in the Calculus of Variations and Nonlinear Elliptic Systems, Princeton University Press, Princeton, NJ, 1983.

[26] R. Glowinski, J. PÉriaux, And G. Terrasson, On the coupling of viscous and inviscid models for compressible fluid flows via domain decomposition, in Third International Symposium on Domain Decomposition Methods for Partial Differential Equations (Houston, TX 1989), SIAM, Philadelphia, 1990, pp. 64-97.

[27] P. Henning and D. Peterseim, Oversampling for the multiscale finite element method, Multiscale Model. Simul., 11 (2013), pp. 1149-1175, https://doi.org/10.1137/120900332.

[28] V. V. Jikov, S. M. Kozlov, And O. A. Oleinik, Homogenization of Differential Operators and Integral Functionals, Springer-Verlag, Berlin, Heidelberg, 1994.

[29] J.-L. Lions, Optimal Control of Systems Governed by Partial Differential Equations, SpringerVerlag, New York, 1971.

[30] J.-L. Lions and E. Magenes, Problèmes aux limites non homogènes et applications, Vol. 1, Travaux et Recherches Mathématiques 1, Dunod, Paris, 1968.

[31] J.-L. Lions and O. Pironneau, Sur le contrôle parallèle des systèmes distribués, C. R. Acad. Sci. Paris Sér. I Math., 327 (1998), pp. 993-998.

[32] A. Målqvis and D. Peterseim, Localization of elliptic multiscale problems, Math. Comp., 83 (2014), pp. 2583-2603, https://doi.org/10.1090/S0025-5718-2014-02868-8.

[33] S. Moskow And M. Vogelius, First-order corrections to the homogenised eigenvalues of a periodic composite medium. A convergence proof, Proc. Roy. Soc. Edinburgh Sect. A, 127 (1997), pp. 1263-1299.

[34] F. Murat and L. Tartar, $H$-convergence, in Topics in the Mathematical Modelling of Composite Materials, Progr. Nonlinear Differential Equations Appl. 31, Birkhäuser Boston, Boston, 1997, pp. 21-43.

[35] J. A. Nitsche And A. H. Schatz, Interior estimates for Ritz-Galerkin methods, Math. Comp., 28 (1974), pp. 937-958.

[36] J. T. Oden And K. S. Vemaganti, Estimation of local modeling error and goal-oriented adaptive modeling of heterogeneous materials. I. Error estimates and adaptive algorithms, J. Comput. Phys., 164 (2000), pp. 22-47, https://doi.org/10.1006/jcph.2000.6585.

[37] D. Olson, P. B. Bochev, M. Luskin, And A. V. Shapeev, An optimization-based atomisticto-continuum coupling method, SIAM J. Numer. Anal., 52 (2014), pp. 2183-2204, https:// doi.org/10.1137/13091734X.

[38] A. Quarteroni And A. Valli, Domain Decomposition Methods for Partial Differential Equations, Numer. Math. Sci. Comput., Oxford Science Publications, The Clarendon Press, Oxford University Press, New York, 1999.

Copyright $@$ by SIAM. Unauthorized reproduction of this article is prohibited. 